LAWRENCE LIVERMORE N A T IO N A L LABORATORY
An Investigation of the Influence of Intial Conditions on Rayleigh-Taylor Mixing

N. J. Mueschke, O. Schilling

November 22, 2004 
This document was prepared as an account of work sponsored by an agency of the United States Government. Neither the United States Government nor the University of California nor any of their employees, makes any warranty, express or implied, or assumes any legal liability or responsibility for the accuracy, completeness, or usefulness of any information, apparatus, product, or process disclosed, or represents that its use would not infringe privately owned rights. Reference herein to any specific commercial product, process, or service by trade name, trademark, manufacturer, or otherwise, does not necessarily constitute or imply its endorsement, recommendation, or favoring by the United States Government or the University of California. The views and opinions of authors expressed herein do not necessarily state or reflect those of the United States Government or the University of California, and shall not be used for advertising or product endorsement purposes.

This work was performed under the auspices of the U.S. Department of Energy by University of California, Lawrence Livermore National Laboratory under Contract W-7405-Eng-48. 


\title{
AN INVESTIGATION OF THE INFLUENCE OF INITIAL CONDITIONS ON RAYLEIGH-TAYLOR MIXING
}

\author{
A Thesis \\ by \\ NICHOLAS J. MUESCHKE
}

\author{
Submitted to the Office of Graduate Studies of \\ Texas A\&M University \\ in partial fulfillment of the requirements for the degree of \\ MASTER OF SCIENCE
}

December 2004

Major Subject: Mechanical Engineering 


\title{
AN INVESTIGATION OF THE INFLUENCE OF INITIAL CONDITIONS ON RAYLEIGH-TAYLOR MIXING
}

\author{
A Thesis \\ by \\ NICHOLAS J. MUESCHKE \\ Submitted to Texas A\&M University \\ in partial fulfillment of the requirements \\ for the degree of \\ MASTER OF SCIENCE
}

Approved as to style and content by:

Malcolm J. Andrews

(Chair of Committee)

\begin{tabular}{c}
\hline $\begin{array}{c}\text { Oleg Schilling } \\
\text { (Member) }\end{array}$ \\
\hline $\begin{array}{c}\text { Hamn-Ching Chen } \\
\text { (Member) }\end{array}$
\end{tabular}

\begin{tabular}{c}
\hline $\begin{array}{c}\text { Ali Beskok } \\
\text { (Member) }\end{array}$ \\
\hline $\begin{array}{c}\text { Dennis O'Neal } \\
\text { (Head of Department) }\end{array}$
\end{tabular}

December 2004

Major Subject: Mechanical Engineering 


\begin{abstract}
An Investigation of the Influence of Initial Conditions on Rayleigh-Taylor Mixing.

(December 2004)

Nicholas J. Mueschke, B.S., University of Louisiana at Lafayette

Chair of Advisory Committee: Dr. Malcolm J. Andrews
\end{abstract}

Experiments and direct numerical simulations (DNS) have been performed to examine the effects of initial conditions on the dynamics of a Rayleigh-Taylor unstable mixing layer. Experiments were performed on a water channel facility to measure the interfacial and velocity perturbations initially present at the two-fluid interface in a small Atwood number mixing layer. The experimental measurements have been parameterized for use in numerical simulations of the experiment. Two- and threedimensional DNS of the experiment have been performed using the parameterized initial conditions. It is shown that simulations implemented with initial velocity and density perturbations, rather than density perturbations alone, are required to match experimentally-measured statistics and spectra. Data acquired from both the experiment and numerical simulations are used to examine the role of initial conditions on the evolution of integral-scale, turbulence, and mixing statistics. Early-time turbulence and mixing statistics are shown to be strongly-dependent upon the early-time transition of the initial perturbation from a weakly-nonlinear to a strongly-nonlinear flow.

UCRL 
To Lea Mueschke 


\section{ACKNOWLEDGMENTS}

The completion of this work is indebted to several individuals. First I would like to thank Dr. Malcolm J. Andrews and Dr. Oleg Schilling for their guidance and support, without whom this body of work would have never left the ground. Thanks are due to the High Energy Density Physics summer student program at Lawrence Livermore National Laboratory for supporting this collaboration, to Dr. Andrew Cook for the use of the MIRANDA code, and to Dr. Schilling for his aid in modifying MIRANDA to incorporate the experimental aspects of this work. Personally, I would like to thank my coworkers Wayne Kraft and Praveen Ramaprabhu, for their aid, intellectual and otherwise. Finally, I wish to thank my wife and parents for supporting my dream to live perpetually as a student. Without their love and support, I would be lost.

This thesis has been written with the aid of Scientific Word 5.0 (copyright MacKichan Software, 2004). All figures and visualizations have been produced using Matlab 6.5 (copyright Mathworks, 2004).

This work was performed under the auspices of the U.S. Department of Energy by the University of California, Lawrence Livermore National Laboratory under Contract No. W-7405-Eng-48. This work has also been supported by the Department of Energy as a part of the High Energy Density Science Grant Program under Contract No. DE-FG03-02NA00060. 


\section{TABLE OF CONTENTS}

1. INTRODUCTION . . . . . . . . . . . . . . . . 1

1.1 Overview . . . . . . . . . . . . . . . . . 1

1.2 Previous Experiments . . . . . . . . . . . . . . 4

1.3 Previous Simulations . . . . . . . . . . . . 7

2. EXPERIMENTAL SETUP AND DIAGNOSTICS . . . . . . 13

2.1 Experimental Facility . . . . . . . . . . . . . . . 13

2.2 Thermocouple Diagnostics . . . . . . . . . . . . . 16

2.3 PIV Diagnostics . . . . . . . . . . . . . . . . . . . . . . . . . . . . . . 22

2.4 PLIF Diagnostics . . . . . . . . . . . . . . 25

3. EXPERIMENTAL RESULTS . . . . . . . . . . . . 31

3.1 Density Measurements . . . . . . . . . . . . . . . . 31

3.2 Velocity Measurements . . . . . . . . . . . . . . . 42

3.3 Spanwise Measurements . . . . . . . . . . . . . . . 45

4. PARAMETERIZATION OF INITIAL CONDITIONS . . . . . 48

4.1 Overview of Parameterization Methods . . . . . . . . 48

4.2 Streamwise Interfacial Perturbation . . . . . . . . . . . 49

4.3 Streamwise Velocity Perturbation . . . . . . . . . . . . 52

4.4 Spanwise Interfacial Perturbation . . . . . . . . . . . . 56

5. DIRECT NUMERICAL SIMULATIONS AND COMPARISONS TO EXPERIMENTAL DATA . . . . . . . . . . . . 57

5.1 Preliminaries . . . . . . . . . . . . . 57

5.2 Numerical Scheme. . . . . . . . . . . . . . . . 58

5.3 Summary of Direct Numerical Simulations . . . . . . . . 60

5.4 Qualitative Results . . . . . . . . . . . . . . . 60

5.5 Integral-Scale Results . . . . . . . . . . . . . . . . 70

5.6 Small-Scale Results . . . . . . . . . . . . . . . . . 74

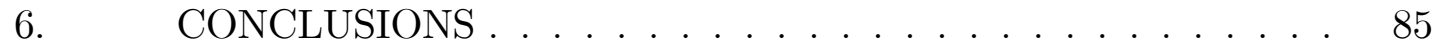

6.1 Experimental Conclusions _. . . . . . . . . . . 85 
6.2 Numerical Conclusions . . . . . . . . . . . . . . . 86

6.3 Future Work . . . . . . . . . . . . . . . 87

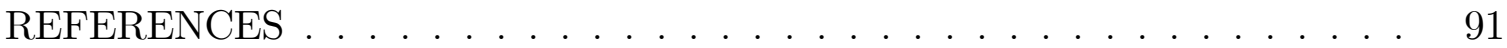

APPENDIX A . . . . . . . . . . . . . . . . . . . . . . . 95

APPENDIX B . . . . . . . . . . . . . . . . . . . 105

APPENDIX C . . . . . . . . . . . . . . . . . . . . . 108

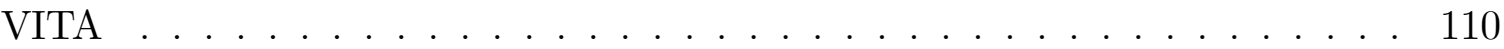




\section{LIST OF FIGURES}

FIGURE

1.1 Illustration of three stages of Rayleigh-Taylor driven mixing: exponential growth of small perturbations (left), saturation of the initial perturbation (middle), "turbulent" regime (right). The images are taken from a three-dimensional DNS with a resolution of $256 \times 128 \times 256 \ldots \ldots \ldots \ldots \ldots \ldots$

2.1 Schematic of the water channel experiment. The conventions for the coordinates and velocities used in the present work are shown on the right-hand side. . . . . . . . . . . . . . . . . . .

2.2 Photograph of water channel experiment. The mean flow is from left-to-right and the top fluid is seeded with a Nigrosene dye. . . . .

2.3 Schematic of the domain measured by the PIV system in the water channel. . . . . . . . . . . . . . . . . . . .

2.4 Schematic of the experimental laser sheet configuration for spanwise PLIF measurements. . . . . . . . . . . . . . . . . . . . . .

2.5 Raw image of warped calibration grid (above) and dewarped calibration grid (below). . . . . . . . . . . . . . . . . .

3.1 Time-evolution of early-time (top) and late-time (bottom) volume fraction PDF, $P\left(f_{1}\right) \ldots \ldots \ldots \ldots \ldots$

3.2 Time-evolution of the molecular mixing quantities, $B_{0}, B_{2}$ and $\theta$. .

3.3 Density power spectrum for $\tau=0.004$ (above left) and $\tau=0.04$ (above right). The compensated spectrum for $\tau=0.004$ exhibits a power-law of $k^{-5}$ (below left) which differs slightly from the power-law of $k^{-6}$ shown at $\tau=0.04$. . . . . . . . . . . . . 


\section{FIGURE}

3.4 Density power spectrum for $\tau=0.19$ (above left) and $\tau=0.39$ (above right). The compensated spectrum for $\tau=0.19$ exhibits a power-law of $k^{-5}$ (below left) which differs from the power-law of $k^{-3}$ shown at $\tau=0.39$ (below right). . . . . . . . . . . . . .

3.5 Density power spectrum for $\tau=0.72$ (above left) and $\tau=1.12$ (above right). The compensated spectrum for $\tau=0.72$ (below left) and $\tau=1.12$ (below right) exhibit an apparent power-law of $k^{-3}$.

3.6 Density power spectrum for $\tau=1.46$ (above left) and $k^{-3}$ compensated spectrum (below right). The $k^{-5 / 3}$ compensated spectra for $\tau=1.12$ (above right) and $\tau=1.46$ (below right) exhibit less than one decade of modes that apparently scale as $E_{\rho}(k) \sim k^{-5 / 3}$. . .

3.7 Field of $w_{r m s}$ at downstream locations $x=0-2.5 \mathrm{~cm} . \ldots . . .$.

3.8 Early-time decay of $w_{r m s}$ in the streamwise direction following the trailing edge of the splitter plate with the channel in a nonbuoyant configuration. . . . . . . . . . . . . .

3.9 Spectrum of $w$ fluctuations at $x=0.5 \mathrm{~cm}$ from the splitter plate. . . 44

3.10 The variation of $\langle u(z)\rangle$ at $\tau=0.018$ for the experiment with the water channel in a non-buoyant configuration. . . . . . . . . . . . . . 46

3.11 PLIF image of spanwise interfacial perturbation. . . . . . . . . . . 47

3.12 Spectrum of spanwise interfacial perturbations. . . . . . . . . . . 47

4.1 Illustration of the initial velocity potential field (top), $u$ velocity field (middle), and $w$ velocity field (bottom). All images are from a $256 \times 256$ DNS with domain size of $16 \mathrm{~cm} \times 16 \mathrm{~cm} . . . . . .$.

5.1 Evolution of the density field from a two-dimensional DNS with initial velocity perturbations. The red and blue represent the two unmixed fluids and intermediate shades represent mixed fluids. Images are shown at $\tau=0.152$ (top left), $\tau=0.758$ (top right), $\tau=1.36$ (bottom left), and $\tau=1.97$ (bottom right). . . . . . . . 
FIGURE

5.2 Evolution of the $f_{1}=0.5$ isosurface from a three-dimensional DNS with initial velocity and interfacial perturbations. Images are shown at $\tau=0.304$ (top) and $\tau=0.607$ (bottom). . . . . . . . .

5.3 Evolution of the $f_{1}=0.5$ isosurface from a three-dimensional DNS with initial velocity and interfacial perturbations. Images are shown at $\tau=0.910$ (top) and $\tau=1.21$ (bottom). . . . . . . . .

5.4 Comparison of slice of (a) PLIF image from experiment and PLIFrendered slices of density field from (b) 3D initial velocity/density conditions DNS and (c) 2D initial velocity conditions DNS at $\tau \approx$ 1.2. All images are approximately $7 \mathrm{~cm} \times 5.25 \mathrm{~cm}$. Experimental image courtesy of W. N. Kraft. . . . . . . . . . . . . . . .

5.5 Time-evolution of the centerplane density (left column) and vertical velocity fields (right column). Images from top to bottom are

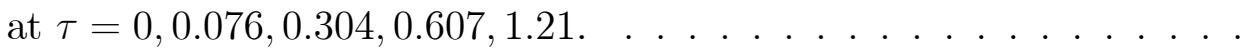

5.6 Time-evolution of the bubble and spike fronts, $h_{b}$ and $h_{s}$, from two- and three-dimensional DNS. . . . . . . . . . . . . . . .

5.7 Time-evolution of the mixing layer width $h$ with respect to nondimensional time. . . . . . . . . . . . . . . . . . . .

5.8 Time-evolution of the integral scale Reynolds number $R e^{c}$ based on terminal velocity of a bubble. . . . . . . . . . . . . . . . .

5.9 Time-evolution of the molecular mixing fraction $\theta$ at the centerplane from various DNS and experimentally-measured values. . . . .

5.10 Time-evolution of $E_{\rho}\left(k_{2 D}\right)$ (top) and $E_{w}\left(k_{2 D}\right)$ (bottom) from a two-dimensional DNS with initial velocity perturbations. . . . . . .

5.11 Time-evolution of $E_{\rho}\left(k_{2 D}\right)$ (top)and $E_{w}\left(k_{2 D}\right)$ (bottom) from a three-dimensional DNS with initial velocity and interfacial per-

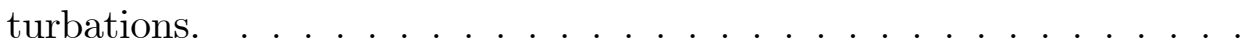

5.12 Compensated energy spectra showing the lack of a $k^{-5 / 3}$ inertial range scaling for $E_{\rho}\left(k_{2 D}\right)$ (top) and $E_{w}\left(k_{2 D}\right)$ (bottom) from a two-dimensional DNS with initial velocity perturbations. . . . . . . 
FIGURE

5.13 Compensated energy spectra showing the lack of a $k^{-5 / 3}$ inertial range scaling for $E_{\rho}\left(k_{2 D}\right)$ (top) and $E_{w}\left(k_{2 D}\right)$ (bottom) from a three-dimensional DNS with initial velocity and interfacial perturbations. 79

5.14 Time-evolution of the density spectrum from a two-dimensional DNS with initial velocity perturbations at: (a) $\tau=0.19$; (b) $\tau=0.39 ;$ (c) $\tau=0.72$, and; (d) $\tau=1.12$. Experimentallymeasured density spectra at equivalent times are shown in black. . .

5.15 Time-evolution of the density spectrum from a three-dimensional DNS with initial velocity and interfacial perturbations at: (a) $\tau=0.19$; (b) $\tau=0.39 ;$ (c) $\tau=0.72$, and; (d) $\tau=1.12$. Experimentally-measured density spectra at corresponding times are shown in black. . . . . . . . . . . . . . . 83

A.1 Schematic of a perturbed interface between two fluids or scalars. . . . 96

A.2 Representative cases of centerline scalar traces for completely unmixed scalars (solid line) and completely mixed scalars (dashed line). . . . . . . . . . . . . . . . . . . . 99 9

A.3 Centerline scalar trace $\phi(x)$ for various values of $L / \lambda \ldots \ldots$. . . . . 98

A.4 Variation of $B_{0}, B_{2}$, and $\theta$ for a range of $L / \lambda \ldots \ldots$. . . . . 98

A.5 Thermocouple probe volume response function for a probe radius

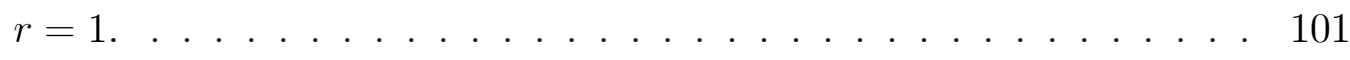

A.6 The resulting centerline scalar trace (dashed line) and actual scalar trace (solid line). . . . . . . . . . . . . . . . . 101

A.7 Correction curves for $L_{m}$ with $r$ as a parameter. . . . . . . . . . 103

C.1 Schematic of the entrance plenum showing egg-crate grill and splitter plate. The screen meshes are not shown. . . . . . . . . . . . 109

C.2 Schematic of boundary layer growth before (left) and after (right) the removal of the plastic ribs adjacent to the splitter plate. . . . . . 109 


\section{LIST OF TABLES}

TABLE Page

1.1 Table of previous simulations and their respective initial conditions .

$2.1 \quad$ Summary of physical and experimental parameters. . . . . . . . . 16

4.1 Minimum and maximum wavenumbers for a typical experiment and for representative two- and three-dimensional DNS. . . . . . . . 52

$5.1 \quad$ Two- and three-dimensional DNS parameters. . . . . . . . . . . . . 61

5.2 Summary of parameterized initial conditions for two-dimensional and three dimensional DNS. . . . . . . . . . . . . . . . . . . . . 61 


\section{INTRODUCTION}

\subsection{Overview}

An experimental and numerical investigation examining the role of initial conditions on buoyancy-driven turbulence has been conducted. In this study, buoyancydriven turbulence was generated by the Rayleigh-Taylor instability, which occurs in an unstable stratification of a heavy fluid with density $\rho_{1}$ above a lighter fluid with density $\rho_{2}$, in a gravitational field $g$ (Rayleigh 1884; Taylor 1950). The RayleighTaylor instability occurs when the density and pressure gradients are oriented such that $\nabla \rho \cdot \nabla p<0$. In the presence of small perturbations at the two-fluid interface, each mode grows exponentially and independently according to linear theory (Chandrasekhar 1961) until nonlinear dynamics begin to dominate the growth of each mode (Haan 1989). Thereafter, modes begin to interact nonlinearly, such that smaller modes merge to create larger buoyant structures. Secondary Kelvin-Helmholtz instabilities grow as localized areas of high shear develop between rising bubbles and falling spikes (Sharp 1984). As the amplitude of a particular mode approaches half of its wavelength, the growth rate of that mode saturates. Finally, as the mixing layer becomes turbulent and grows in spatial extent, dimensional analysis shows that, the only relevant lengthscale is $g t^{2}$, under the appropriate conditions. In this self-similar regime, the width of the mixing layer is modeled as (Anuchina et al. 1978; Youngs 1984)

$$
h=\alpha A g t^{2}
$$

where $A \equiv\left(\rho_{1}-\rho_{2}\right) /\left(\rho_{1}+\rho_{2}\right)$ is the Atwood number and $\alpha$ is dimensionless. However, the determination of the exact conditions under which equation (1.1) holds is a current

This thesis follows the style of the Journal of Fluid Mechanics. 

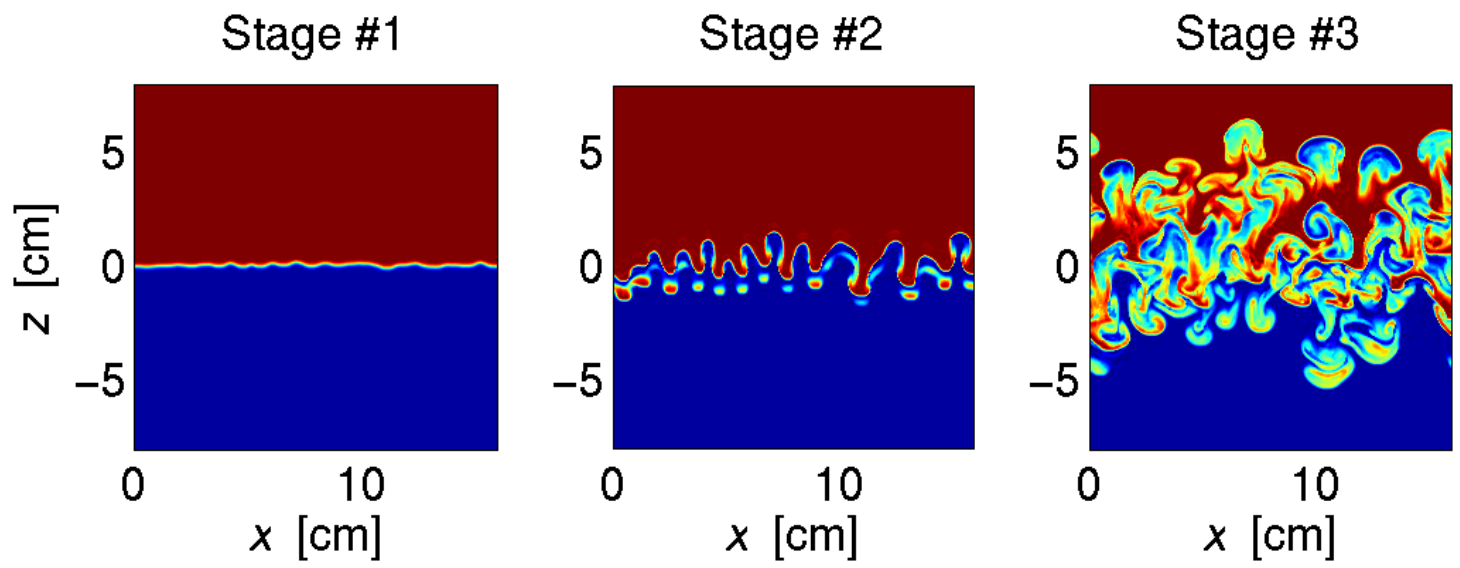

Figure 1.1 Illustration of three stages of Rayleigh-Taylor driven mixing: exponential growth of small perturbations (left), saturation of the initial perturbation (middle), "turbulent" regime (right). The images are taken from a three-dimensional DNS with a resolution of $256 \times 128 \times 256$.

area of research and will be discussed further in $\S 1.2$ and $\S 1.3$. As the instability develops and nonlinear processes begin to dominate, a turbulent mixing layer develops between the two fluids at sufficiently large Reynolds numbers. An illustration of the three stages is shown in figure 1.1.

Rayleigh-Taylor flows represent one of the few canonical fluid flows that encompass the laminar, transitional, and turbulent regimes. The development of a complete understanding of Rayleigh-Taylor instability-generated turbulence is important because of the broad impact such flows have in nature and in technological applications. In astrophysical flows, it is hypothesized that the limiting factor in the creation of heavy elements in collapsing stars is the growth of the mixing layer formed by the adverse stratification of densities in the gravitational field of a star (Smarr et al. 1981). Many deep-sea ocean currents and atmospheric flows contain Rayleigh-Taylor generated mixing and turbulence (Molchanov 2003; Cui \& Street 2004). Rayleigh-Taylor 
instabilities have been found to occur in geophysical applications, such as the interaction between the Earth's crust and mantle layers (Zandt et al. 2004). The break up of fuel droplets in a high-speed flows have also been found to be Rayleigh-Taylor unstable (Thomas 2003; Marmottant \& Villermaux 2004). At much smaller scales, the performance of inertially-confined fusion targets are susceptible to buoyancy- and shock-driven instabilities, in which a core of deuterium and tritium fuel is compressed by high-density shells. The acceleration phase of an inertial confinement fusion (ICF) capsule compression has been shown to be Richtmyer-Meshkov unstable, while the late-time deceleration phase is Rayleigh-Taylor unstable; the Richtmyer-Meshkov instability is the impulsively-driven variant of the Rayleigh-Taylor instability. The growth of a Rayleigh-Taylor driven mixing layer has been shown to be the limiting factor in the effective yield of (ICF) target capsules. (Lindl 1998; Betti et al. 2001; Atzeni \& Meyer-ter-Vehn 2004).

Modeling such complicated, multi-scale flows, which contain a perpetually expanding range of timescales and lengthscales, represents a grand challenge for the turbulence community. Validation of predictive turbulent transport models of anisotropic, inhomogeneous, variable-density turbulence and mixing require a priori knowledge of velocity and density correlations, such as $\overline{u_{i}^{\prime} u_{j}^{\prime}}, \overline{\rho^{\prime} u_{i}^{\prime}}$ and $\overline{u_{i}^{\prime} u_{j}^{\prime} u_{k}^{\prime}}$ (where is the overbar denotes Reynolds averaging), to validate closure models. Currently, there are no turbulence and mixing models that incorporate the effects of initial conditions in their predictions of the development of turbulent quantities during the growth of a Rayleigh-Taylor driven mixing layer.

The principle objectives of the present investigation are to:

- Experimentally measure the initial density and velocity perturbations at the two-fluid interface of a Rayleigh-Taylor mixing layer, including the first mea- 
surements of the spanwise perturbations;

- parameterize the measured initial density and velocity conditions so that they may be implemented in numerical simulations of the experiment;

- perform two- and three-dimensional direct numerical simulations (DNS) of the experiment using an accurate, high-resolution numerical scheme in which all viscous and mass diffusion scales are resolved;

- examine turbulence and mixing statistics from both numerical and experimental results to examine the role of initial conditions in the early-time transition of the mixing layer from a weakly-nonlinear to a strongly-nonlinear flow.

Many experiments and numerical simulations have been performed to determine which factors influence the growth and internal structure of a Rayleigh-Taylor mixing layer, and are reviewed in the following sections. However, the lack of control over the initial seeding of the perturbations and the inability to fully quantify the initial conditions in all directions is common to nearly all experimental designs. Similarly, numerical simulations are either initialized with ad hoc initial perturbations or utilize some form of partially-measured (typically one-dimensional) initial conditions. An overview of the major contributions to the study of Rayleigh-Taylor generated turbulence and mixing will be presented here.

\subsection{Previous Experiments}

The first single-mode Rayleigh-Taylor instability experiments were performed by Taylor (1950) using a vertical tube containing fluids having different densities. Emmons et al. (1960) accelerated a tank on rails to generate an unstable interface between methanol and air. Read (1984) performed the first significant measurements 
of the growth of a Rayleigh-Taylor mixing layer seeded with a multi-mode perturbation using a drop tank accelerated downward by rocket motors. This experiment allowed for high accelerations and a large range of Atwood numbers, but had no measurement of the initial conditions or of the internal structure of the mixing layer. In a similar experiment, Dimonte and Schneider (1996) used the linear electric motor (LEM) facility at the Lawrence Livermore National Laboratory to accelerate a tank, producing an unstable interface between two liquids. While more experimental control and diagnostics were available for the LEM experiments, only a qualitative description and estimate of the initial perturbations was possible.

Other experiments include the overturning of a tank containing two fluids of different densities (Andrews 1986; Andrews \& Spalding 1990). The experiments performed by Andrews included the adverse stratifications of fluids with a nominally flat interface and with tilted interfaces. For the simulations conducted to model the experiment, the initial tilt angle of the two-fluid interface could be accurately measured by optical techniques, but the initial fine-scale perturbations could not be measured. Linden and Redondo (1991), Linden et al. (1994), and Dalziel et al. (1999) produced an unstable interface by withdrawing a plate from a tank that contained a heavier fluid above a lighter fluid. These experiments allowed for a partial quantification of the initial conditions. Dalziel et al. (1999) used a particle tracking method to measure the velocity perturbation introduced by the withdrawal of the splitter plate in a two-dimensional plane. Numerical simulations performed by Dalziel et al. will be reviewed in the following section.

Snider and Andrews (1994) developed a water channel device similar to many shear layer experiments. Two fluid streams were initially separated by a thin splitter plate where both stream velocities are matched, unlike in the shear layer arrangement. The same water channel was used in this current investigation and is discussed 
in detail in $§ 2.1$ and shown in figure 2.1. Snider and Andrews used optical techniques to measure the self-similar quadratic growth of the mixing layer in time, with a measured value of $\alpha=0.07 \pm 0.011$. Snider and Andrews also used optical techniques to measure the average volume fraction profile across the width of the mixing layer. Wilson (2002) and Wilson and Andrews (2002) also used the same water channel to investigate the internal structure of fluctuating quantities and the degree of molecular mixing within a Rayleigh-Taylor mixing layer. Wilson and Andrews (2002) used thermocouples to measure the average volume fraction and the degree of molecular mixing across the mixing layer at the early- and late-time stages within the mixing layer development. The use of thermocouples also allowed for the measurement of the density energy spectra. Wilson and Andrews reported a $k^{-5 / 3}$ power-law in the intermediate-wavenumber range and a $k^{-3}$ power-law in the high-wavenumber range at later times. Wilson (2002) used particle image velocimetry (PIV) to measure the turbulent velocity correlations $\overline{u^{\prime 2}}, \overline{w^{\prime 2}}$ and $\overline{u^{\prime} w^{\prime}}$ for both buoyancy-driven and combined buoyancy/shear-driven mixing layers, where $u$ and $w$ are the streamwise and vertical velocity component, respectively, and $u^{\prime}$ and $w^{\prime}$ are the fluctuating components of $u$ and $w$

$$
u^{\prime}=u-\bar{u} ; w^{\prime}=w-\bar{w}
$$

Due to extended data collection times, statistical convergence of the velocity and density spectra, as well as of double and triple correlations, was achieved. Ramaprabhu (2003) and Ramaprabhu and Andrews (2004a) used the same water channel configuration to measure both large-scale and small-scale statistics, such as the components of the Reynolds stress anisotropy tensor (Pope 2000)

$$
b_{i j} \equiv \frac{\overline{u_{i}^{\prime} u_{j}^{\prime}}}{\overline{u_{k}^{\prime 2}}}-\frac{\delta_{i j}}{3},
$$


where

$$
\delta_{i j}=\left\{\begin{array}{lll}
1 & \text { if } & i=j \\
0 & \text { if } & i \neq j
\end{array}\right.
$$

is the Kronecker tensor. Ramaprabhu and Andrews (2003) used PIV-S (scalar) to simultaneously measure a two-dimensional velocity field and the density field, resulting in measurements of $\overline{\rho^{\prime} u^{\prime}}$ and $\overline{\rho^{\prime} w^{\prime}}$. Of particular interest are the Ramaprabhu and Andrews (2004a; 2004b) measurements of the initial density and velocity fluctuations at the two-fluid interface of a Rayleigh-Taylor mixing layer. The numerical simulations that implemented the one-dimensional (in wavenumber space) measurements will be reviewed in the following section.

\subsection{Previous Simulations}

Youngs (1984) performed monotone-integrated large eddy simulations (MILES) (Boris et al. 1992; Pope 2000) of two-dimensional, incompressible, miscible RayleighTaylor instability-generated turbulence using the Eulerian hydrodynamics code TURMOIL. The MILES method represents a class of numerical methods that solves the Euler equations, but incorporates an effective viscosity term through numerical truncation errors. Small-scale fluctuations are damped by numerical diffusion, which is dependent upon the numerical grid resolution. Youngs' early simulations were aimed at determining the growth of single- and multi-mode perturbations. He showed that simulations implemented with only high-wavenumber velocity perturbations did not grow as fast in the later stages of development as simulations that also included a low-wavenumber interfacial perturbation. Youngs (1991) subsequently performed a three-dimensional ( $128^{3}$ grid points) simulation with isotropic, multi-mode interfacial perturbations. Youngs continued the theme of examining the late-time growth rate of the mixing layer with respect to the determination of an asymptotic value of $\alpha$ 
in equation (1.1). Youngs also addressed the issue of the structure and evolution of internal fluctuating quantities, in particular, density fluctuations and molecular mixing. Youngs (1994) presented similar findings with a higher resolution $\left(160^{2} \times 270\right)$ simulation.

Using the same numerical method as Youngs (1991; 1994), Linden et al. (1994) and Dalziel et al. (1999) performed a three-dimensional simulation $(160 \times 80 \times 200)$ of the plate-withdrawal experiment described previously. Linden et al. (1994) used short-wavelength, isotropic interfacial perturbations with the addition of a single, long-wavelength interfacial perturbation in the $x$-direction to simulate the platewithdrawal. Dalziel et al. also showed that an irrotational (potential flow) model of the initial velocity disturbance generated better agreement between experiments and simulations than the use of interfacial perturbations alone. Also, both simulations (Linden et al. 1994; Dalziel et al. 1999) implemented pseudo-anisotropic initial conditions to simulate the experiment, where either a single-wavelength interfacial perturbation or a two-dimensional velocity field was superimposed onto a flow with isotropic perturbations. However, several factors prevented a direct comparison between experiment and the simulation. The experiments were performed with Atwood numbers $A=1 \times 10^{-4}$ to $5 \times 10^{-2}$ (Linden et al. 1994 ) and $A=2.0 \times 10^{-3}$ (Dalziel et al. 1999), while the simulations implemented $A=9.1 \times 10^{-2}$ for numerical stability reasons. Also, the numerical simulations had an effective numerical Schmidt number, $S c \equiv \nu / D \sim 1$, while the experiments had $S c \sim 1000$, where $\nu$ is the kinematic viscosity and $D$ is the diffusivity of mass between the two fluids. Finally, the three-dimensional numerical simulations did not include any measured perturbations in the spanwise direction. With this method of initialization it is not clear how the initial seeding in the second homogeneous direction affects the early-time structure and transition of the mixing layer to a strongly-nonlinear flow and a self-similar state 
at later times.

More recently, Cook \& Dimotakis (2001; 2002) performed $256^{2} \times 1024$ DNS of Rayleigh-Taylor mixing with $A=0.5$ and $S c=1$ to examine how initial conditions affect the asymptotic growth of the mixing layer and the evolution of the degree of molecular mixing within the layer. In contrast to the MILES technique, DNS solves the full Navier-Stokes equations, resolving all length- and timescales. DNS is severely limited by the number of computational gridpoints and computing resources available for a given simulation. The Cook and Dimotakis simulations implemented isotropic, broad-banded interfacial perturbations within an annulus of wavenumbers, assuming a Gaussian distribution about a particular mode number of interest. The same numerical scheme was used with an increased resolution of $512^{2} \times 2040$ to examine the transfer of energy between scales of motion (Cook \& Zhou 2002; Cabot et al. 2004). Under the assumption that the memory of the initial conditions can be neglected in the late-time, self-similar growth regime, Cook et al. (2004) used a very high-resolution large-eddy simulation $\left(1152^{3}\right)$ to further investigate the asymptotic growth of a mixing layer and the late-time mixing transition (Dimotakis 2000).

Large-eddy simulation (LES) techniques solve a filtered form of the Navier-Stokes equations where only the large and intermediate scales of motion are resolved. The effects of the unresolved scales are modeled by a second-order residual subgrid-scale Reynolds stress tensor $\tau_{i j}^{R} \equiv \overline{U_{i} U_{j}}-\overline{U_{i}} \overline{U_{j}}$ where the overline denotes filtering in this case (Smagorinsky 1963; Lilly 1967; Pope 2000). In wavenumber space, the modelling of $\tau_{i j}^{R}$ is accomplished by the calculation or modeling of triadic wave interactions between resolved and filtered fluctuations (Leslie \& Quarini 1979; Pope 2000; Cabot et al. 2004). This method has the advantage of greater computational efficiency and achieving a greater range of Reynolds numbers than suitable for DNS. The LES performed by Cook et al. (2004) implemented isotropic, narrow-banded interfacial 
perturbations with a Gaussian distribution about a particular mode.

Dimonte et al. (2004) also studied how the initial conditions affect the asymptotic growth rate of a Rayleigh-Taylor mixing layer. Dimonte et al. performed a study employing a variety of numerical schemes using spatial resolutions of $128^{2} \times 256$ and $256^{2} \times 512$. The initial interfacial perturbations were initialized with an isotropic, approximately uniform distribution of energy in either modes 16-32 or 32-64 so that all of the simulations would evolve in the limit of strong mode-coupling. Dimonte et al. demonstrated a lower-bound value of $\alpha \approx 0.03$ when mode-coupling is the only mechanism for the development of larger scales.

Ristorcelli and Clark (2004) performed an ensemble of simulations $\left(150^{2} \times 300\right)$ of a Rayleigh-Taylor mixing layer using pseudo-spectral DNS techniques to investigate the temporal evolution and self-similarity of many fluctuating quantities within the mixing layer. As the late-time, self-similar behavior of the mixing layer was of interest, an ad hoc initial Gaussian distribution of modes was used for the initial conditions of the DNS. Multiple simulations were performed to examine the evolution and collapse of turbulence and mixing statistics in the self-similar regime. Ristorcelli and Clark showed that many statistical quantities measured across the mixing layer at late times collapse onto a single curve when normalized by the mixing layer width.

Ramaprabhu and Andrews (2004b) used measured density and velocity fluctuations to perform numerical simulations of the water channel experiment (Ramaprabhu \& Andrews 2004a). Ramaprabhu and Andrews (2004b) used a MILES code (Andrews 1986; Ramaprabhu 2003) to investigate how well numerical simulations initialized with density and velocity perturbations agree with experimentally-measured mixing layer growth rates and fluctuating quantities. Instead of implementing the onedimensional measurement in one direction within the simulation and assuming a perturbation in the other direction like Dalziel et al. (1999), they assumed isotropic initial 


\begin{tabular}{ccc}
\hline Researcher & Isotropy & Initial Conditions \\
\hline \hline Youngs (1991) & Isotropic & Interfacial (ad hoc) \\
Youngs (1994) & Isotropic & Interfacial (ad hoc) \\
Linden et al. (1994) & Pseudo- & Interfacial (ad hoc) \\
Dalziel et al. (1999) & anisotropic & \\
Cook \& Dimotakis (2001) & Pseudo- & Velocity (measured) and \\
Cook \& Zhou (2003); Cabot et al. $(2004)$ & Isotropic & Interfacial (ad hoc) \\
Cook et al. $(2004)$ & Isotropic & Interfacial (ad hoc) \\
Ristorcelli \& Clark (2004) & Isotropic & Interfacial (ad hoc) \\
Ramaprabhu \& Andrews (2004b) & Isotropic & Interfacial (ad hoc) \\
Ramaprabhu \& Andrews (2004b) & Isotropic & Velocity (measured) \\
Dimonte et al. (2004) & Isotropic & Interfacial (ad hoc) \\
Mueschke (2004) & Anisotropic & Velocity (measured) and \\
& & Interfacial (measured) \\
\hline
\end{tabular}

Table 1.1 Previous and current three-dimensional simulations and their respective initial conditions.

perturbations in all homogeneous directions. The three-dimensionality of the flow was seeded by rotating the one-dimensional density or velocity spectrum in wavenumber space to create a two-dimensional perturbation. It was found that simulations with velocity perturbations matched closer with experimental measurements of growth rates and fluctuating quantities than simulations using interfacial perturbations alone.

A summary of previous simulations is shown in table 1.1. The objectives of the present work differ from the majority of the previous research involving Rayleigh- 
Taylor flows; this research extends some of the previous investigations by including a proper method for measuring and implementing initial conditions of a Rayleigh-Taylor flow. The work presented in this thesis extends the work performed by previous researchers in the following ways: it is the first to implement fully measured, anisotropic initial velocity and interfacial perturbations in a numerical simulation. Also, this investigation is the first use of DNS to simulate the turbulent mixing layer formed in the water channel experiment. 


\section{EXPERIMENTAL SETUP AND DIAGNOSTICS}

\subsection{Experimental Facility}

The experimental objectives of this research have been accomplished using an existing water channel facility at Texas A\&M University (Snider \& Andrews 1994; Wilson \& Andrews 2002; Ramaprabhu \& Andrews 2004a). The water channel is an open-loop device in which cold and warm water $\left(\Delta T \approx 5^{\circ} \mathrm{C}\right)$ enter the channel initially separated by a thin Plexiglas splitter plate. A schematic of the water channel is shown in figure 2.1. The density difference between the two streams is induced by the thermal expansion of the warmer fluid. The water channel is supplied by two 500-gallon water tanks and has a running time of approximately 10 minutes. Sump pumps in each tank ensure adequate stirring of the water to maintain temperature uniformity. Cold and hot water supplies are pumped into the entrance plenum of the water channel where each stream flows through an arrangement of flow-straighteners, followed by a series of screen meshes $(30 \times 30$ wires/in). A description of enhancements made to the screen meshes is given in Appendix C. The screen meshes are intended to eliminate free-stream velocity fluctuations and reduce the momentum deficit caused by boundary layers along the walls of the channel and splitter plate. The splitter plate terminates in a $2.5^{\circ}$ knife-edge followed immediately by another screen mesh $(35 \times 35$ wires/in). The final screen mesh ("end-screen") is intended to minimize the momentum deficit at the trailing edge of the splitter plate, thereby reducing the magnitude and wavelength of shedding vortices.

Upon entering the mixing section of the channel, an adverse density stratification occurs between the two water streams, inducing Rayleigh-Taylor mixing downstream. The mixing section of the water channel is $100 \mathrm{~cm}$ long ( $x$-direction) with crosssectional dimensions of $20 \mathrm{~cm} \times 32 \mathrm{~cm}$ (width $\times$ height). In the present experiments, 


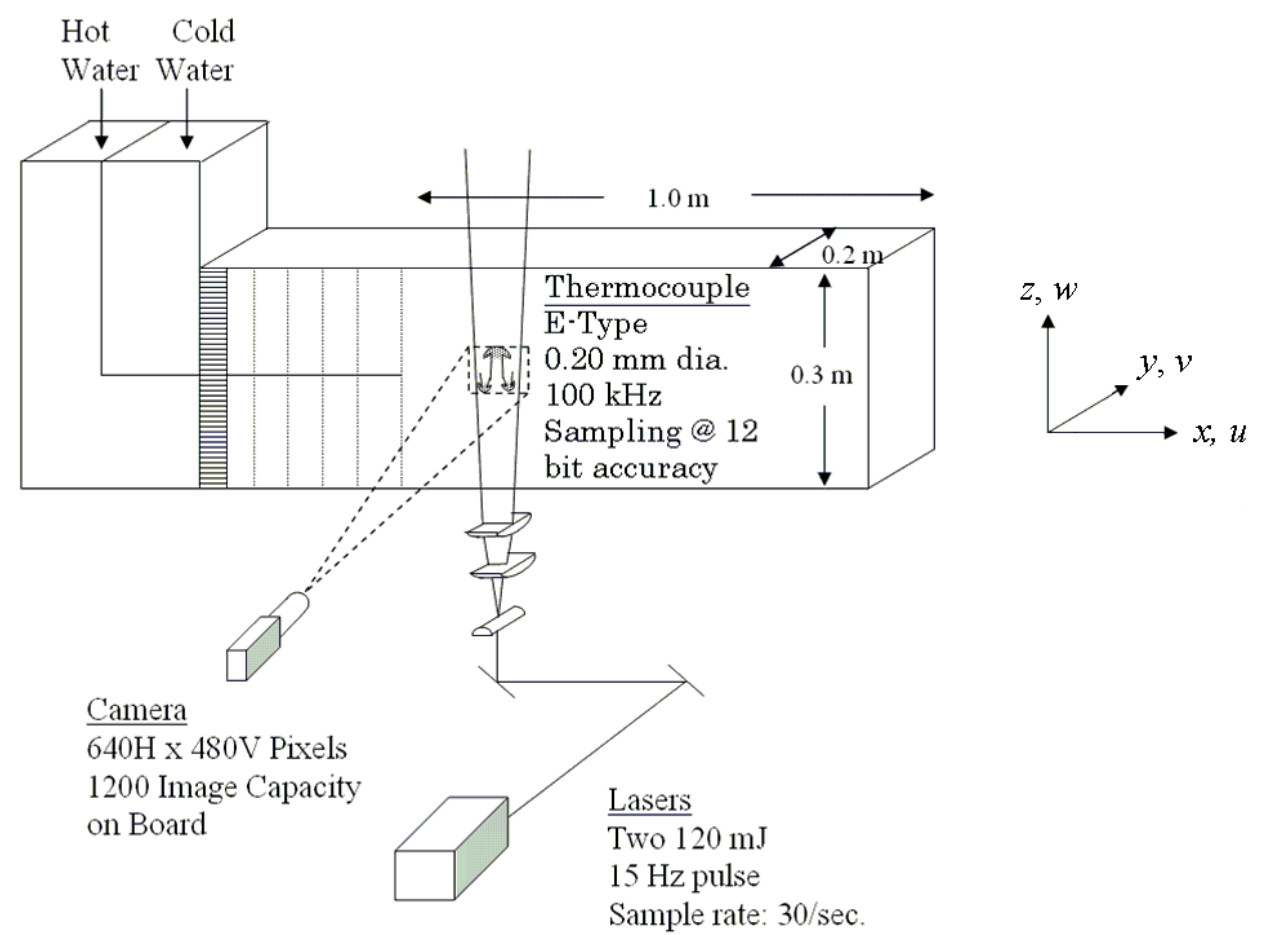

Figure 2.1 Schematic of the water channel experiment. The conventions for the coordinates and velocities used in the present work are shown on the right-hand side.

the mean advective velocity $U_{m}$ of the two stream velocities are matched $\left(U_{m} \approx 4.2\right.$ $\mathrm{cm} / \mathrm{s}$ ) so that no shear due to mean velocity gradients exists. The sidewalls have been shown by previous researchers to have negligible influence on the growth rate of the mixing layer (Snider \& Andrews 1994). The water channel measurements have also been shown to be statistically-stationary for higher-order moments of velocity fluctuations and density fluctuations (Wilson 2002; Ramaprabhu 2003). Figure 2.1 shows a schematic of the water channel and its diagnostic capabilities.

To illustrate the spatial and temporal development of the mixing layer within the water channel, an image of the mixing layer is shown in figure 2.2. Nigrosene dye was added to the top stream for visualization purposes. Small perturbations, which are 


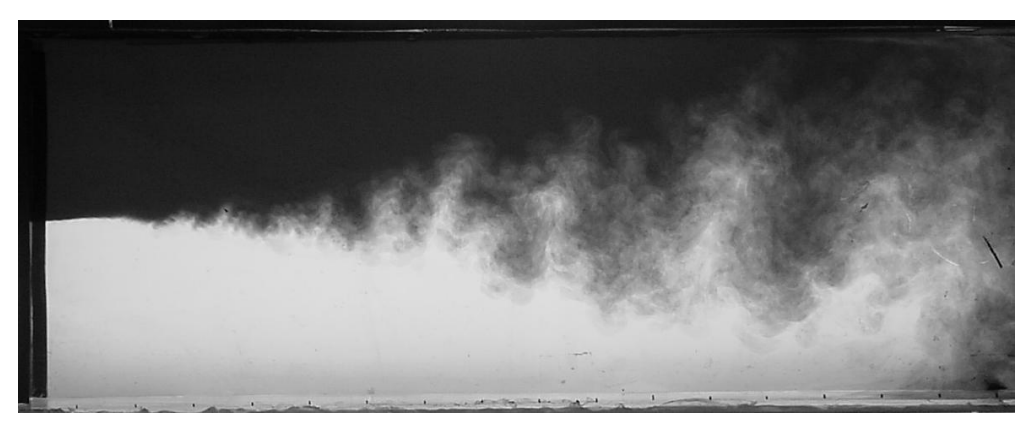

Figure 2.2 Photograph of water channel experiment. The mean flow is from leftto-right and the top fluid is seeded with a Nigrosene dye.

sub-pixel in size in the image shown, grow and interact to form the mixing layer that is seen on the right-hand side of the image. Downstream distance is converted to time by Taylor's hypothesis, such that $t=x / U_{m}$, where $x$ is the downstream position of the thermocouple probe from the trailing edge of the splitter plate (Taylor 1938; Pope 2000). Time is normalized as in Dalziel et al. (1999) and Ramaprabhu \& Andrews (2004a):

$$
\begin{aligned}
\tau & \equiv t \sqrt{\frac{A g}{H}} \\
& =\frac{x}{U_{m}} \sqrt{\frac{A g}{H}}
\end{aligned}
$$

where $H=32 \mathrm{~cm}$ is the height of the mixing layer channel. A summary of the experimental parameters is given in table 2.1 .

One goal of the present investigation is to measure the initial velocity and interfacial perturbations of the mixing layer so that their influence on the development of the mixing layer can be determined. To accomplish this, three separate and independent measurements were performed to quantify the initial conditions of the flow. First, the fluctuating density field off the trailing-edge of the splitter plate was measured using a high-resolution thermocouple. Velocity perturbations in the streamwise 


\begin{tabular}{|c|c|c|}
\hline Parameter & Value & Description \\
\hline$\rho_{1}$ & $0.9986 \mathrm{~g} / \mathrm{cm}^{3}$ & Approximate density of top stream \\
\hline$\rho_{2}$ & $0.9970 \mathrm{~g} / \mathrm{cm}^{3}$ & Approximate density of bottom stream \\
\hline$A$ & $7.5 \times 10^{-4}$ & Atwood number \\
\hline$g$ & $-981 \mathrm{~cm} / \mathrm{s}^{2}$ & Gravity \\
\hline$U_{m}$ & $4.2 \mathrm{~cm} / \mathrm{s}$ & Mean advective velocity \\
\hline$\mu_{1}$ & $0.009 \mathrm{~g} / \mathrm{cm} \mathrm{s}$ & Dynamic viscosity of top stream \\
\hline$\mu_{2}$ & $0.011 \mathrm{~g} / \mathrm{cm} \mathrm{s}$ & Dynamic viscosity of bottom stream \\
\hline $\operatorname{Pr}$ & 7.0 & $\begin{array}{l}\text { Prandtl number } \operatorname{Pr} \equiv \nu / \alpha \\
\nu=\left(\mu_{1}+\mu_{2}\right) /\left(\rho_{1}+\rho_{2}\right) \\
\alpha \text { is the thermal diffusivity }\end{array}$ \\
\hline
\end{tabular}

Table 2.1 Summary of physical and experimental parameters.

direction were measured using particle image velocimetry (PIV). Finally, the interfacial perturbations in the spanwise direction were measured using planar laser-induced fluorescence (PLIF). Further details of each experimental method are given below.

\subsection{Thermocouple Diagnostics}

The measurement of the interfacial perturbation in the streamwise $(x)$ direction has been accomplished using a new high-resolution, E-type thermocouple measurement system. Temperature fluctuations were measured using thermocouples positioned at $x=0.1$ and $x=1.0 \mathrm{~cm}$ downstream from the splitter plate. The new thermocouples are constructed of 40 gauge wire ( $0.08 \mathrm{~mm}$ diameter) with a weld bead diameter of 0.16 $\mathrm{mm}$. They have a smaller weld bead diameter than the thermocouples previously used in water channel experiments, resulting in a less intrusive diagnostic and improved 
spatial resolution. The spatial resolution enhancements reduce the uncertainty in the mixing statistics and scalar fluctuation spectra. Additional details on the spatialaveraging of temperature measurements due to probe volume size can be found in Appendix A. The thermocouple wire, extension wire, and all connections have been shielded and grounded to minimize extraneous EMI and RFI noise. Temperature measurements were recorded at a rate of $50 \mathrm{kHz}$ using a 16-bit data acquisition (DAQ) system. Spurious noise from the temperature trace was eliminated using a 100-point averaging window, reducing the sampling rate to an effective $500 \mathrm{~Hz}$. Using Taylor's hypothesis, at this sampling rate and a mean advective velocity of $U_{m} \approx 4.2 \mathrm{~cm} / \mathrm{s}$, the temperature measurements are separated by $0.084 \mathrm{~mm}$, which is approximately half the diameter of the probe volume. Fluctuations in the temperature measurements at the Nyquist frequency $(250 \mathrm{~Hz})$ were found to be below the level of system noise, obviating the need for a more complicated filtering algorithm that retains spectral resolution.

In addition to accurately measuring temperature data at several downstream positions, another measurement was made to accurately determine the time of development of the mixing layer $\tau$. To ensure an accurate measurement of $\tau$, three measurements were taken. First, the distance between the splitter plate and probe volume was carefully measured. Second, the Atwood number was measured accurately. Finally, the mean flow velocity of the water channel was measured accurately, which is an important detail with respect to conducting thermocouple experiments. The following procedure was devised so that an accurate mean velocity measurement could be made. First, the mean advective velocity for both streams was adjusted until no shear was present and $U_{m} \approx 4.1-4.5 \mathrm{~cm} / \mathrm{s}$. The presence of a mean velocity gradient between the two streams was determined by injecting dye on the splitter plate just before the end-screen. Once the dye was entrained into the mixing layer, 
it was possible to examine the "lean" of the developing Rayleigh-Taylor structures at downstream locations. In the absence of any leaning, the difference between the top and bottom stream velocities was shown to be $\left|U_{1}-U_{2}\right|<0.2 \mathrm{~cm} / \mathrm{s}$ (Ramaprabhu 2003). To measure the mean advective velocity, Nigrosene dye was injected into the cold water stream at approximately $25 \mathrm{~cm}$ before the end of the splitter plate. Images of the mixing section of the water channel $(4.25 \mathrm{~cm} \times 3.19 \mathrm{~cm} ; 640 \times 480$ pixels $)$ were recorded at $30 \mathrm{~Hz}$ using $L a b V I E W^{1}$ and a Kodak Megaplus ${ }^{2}$ digital imaging system. The mean advective velocity $U_{m}$ was calculated by dividing the width of the imaging frame $L_{x}$ by the time required for the fragment of dye to completely traverse the imaging plane, where

$$
U_{m}=\frac{30 L_{x}}{N_{\text {frames }}}
$$

and $N_{\text {frames }}$ is the number of frames required for the dye tracer to traverse $L_{x}$. The factor of 30 in equation (2.2) arises from the sampling rate of the camera. Care was taken to inject the dye at the same focal distance as $L_{x}$ was measured.

Temperature measurements were converted to density values using an equation of state for water (Kukulka 1981):

$$
\rho(T)=\frac{P(T)}{Q(T)},
$$

where $P(T)$ and $Q(T)$ are polynomials defined as:

$$
\begin{aligned}
& P(T)=999.8396+12.2249 T-0.007922 T^{2}-55.448 \times 10^{-6} T^{3} \\
& +149.756 \times 10^{-9} T^{4}-393.295 \times 10^{-12} T^{5}, \\
& Q(T)=1+18.159 \times 10^{-3} T
\end{aligned}
$$

\footnotetext{
${ }^{1}$ LabVIEW is a trademark of National Instruments Corporation.

${ }^{2}$ MegaPlus is a trademark of Eastman Kodak Company.
} 
in units of $\mathrm{kg} / \mathrm{m}^{3}$.

The thermocouple was mounted on a vertical rake that allowed for fine adjustment of the probe location in the vertical $(z)$ direction. All measurements in this study have been conducted on the "centerplane" of the mixing layer, which is defined as the plane in which equal portions of fluid 1 and fluid 2 exist. Mathematically, the centerplane is defined by the location in which the average volume fraction of the $i^{\text {th }}$ fluid is $\overline{f_{i}}=1 / 2$ for the two-fluid case. The average volume fraction of fluid 1 is defined by the time average

$$
\overline{f_{1}}=\frac{1}{\tau} \int_{0}^{\tau} \frac{\rho(t)-\rho_{2}}{\rho_{1}-\rho_{2}} d t
$$

and $\overline{f_{1}}+\overline{f_{2}}=1$.

To determine the location of the centerplane, a series of temperature measurements were recorded at $200 \mathrm{~Hz}$ and the time-averaged volume fraction for fluid 1, $\overline{f_{1}}$, was evaluated. Depending upon the value of $\overline{f_{1}}$, the position of the probe was adjusted vertically until $\overline{f_{1}}=0.50 \pm 0.025$. Once the probe was located on the centerplane, a two-minute interval of temperature measurements was recorded at $50 \mathrm{kHz}$. This allows for the passage of an adequate number of large-scale (long-wavelength) perturbations and enforces statistical convergence criteria. Further details on the statistical convergence of temperature measurements can be found in Wilson and Andrews (2002). A detailed description of experimental enhancements with respect to this procedure can be found in Appendix B.

In addition to the measurements at $x=0.1 \mathrm{~cm}$ and $x=1.0 \mathrm{~cm}$, density measurements were taken at several downstream locations to investigate the evolution of various density statistics. All measurements were taken on the centerplane of the mixing layer, satisfying the criteria that $\overline{f_{i}}=1 / 2$. However, the average volume fraction provides no information regarding the distribution of density values at a par- 
ticular downstream location. This information is provided in the probability density function of the volume fraction trace $P\left(f_{1}\right)$, defined in its discrete form by

$$
P\left(f_{1}\right)=\frac{F\left(f_{1}\right)}{N_{\text {samp }} \delta_{\text {bin }}}
$$

where $F\left(f_{1}\right)$ is the frequency distribution of $f_{1}, N_{\text {samp }}$ is the number of samples, and $\delta_{b i n}$ is the bin width of $F\left(f_{1}\right)$.

The range and distribution of scales present in the fluctuating component of the density field are also of interest. This information is determined by calculating the power spectrum of the density trace. First, the one-dimensional Fourier transform of the fluctuating density trace is calculated by

$$
\begin{aligned}
\widehat{\rho}(k) & =\mathcal{F}\left[\rho(x)^{\prime}\right] \\
& =\int_{0}^{L} \rho(x)^{\prime} \mathrm{e}^{-i k x} d x,
\end{aligned}
$$

where $\mathcal{F}$ denotes the Fourier transform, $k \equiv 2 \pi / \lambda$ is the wavenumber, and $\rho(x)^{\prime}=$ $\rho(x)-\langle\rho\rangle$ defines the fluctuating density trace. The mean density value $\langle\rho\rangle$ is defined by the spatial-averaging operator

$$
\langle\phi\rangle=\frac{1}{L} \int_{0}^{L} \phi(x) d x,
$$

where $\phi$ is any scalar variable, $L \equiv U_{m} N_{\text {samp }} / f_{\text {samp }}$ is the distance over which $\phi(x)$ was measured, $N_{\text {samp }}$ is the number of samples, and $f_{\text {samp }}$ is the sampling rate in Hz. Note that the density field within the experiment is a function of all spatial dimensions and time $\rho(x, y, z, t)$, but the density trace used in the spectral analysis is only a function of the $x$ coordinate. This is due to the fact that measurements were taken at a fixed point in space, with time being the independent parameter. The change of variables from temporal variation to spatial variation utilizes Taylor's 
hypothesis to relate $\rho(t)$ and $\rho(x)$, allowing for spatial-averaging to be performed, rather than temporal-averaging. Due to the fact that the probe volume records a one-dimensional measurement, the dependence of the density fields upon the other spatial and temporal dimensions has been dropped. The discrete wavenumbers are

$$
k_{m}=\frac{2 \pi m}{L}
$$

where $m$ is the mode number. Equation (2.8) gives the amplitudes and phase in complex space of the fluctuations for each wavenumber from which the one-dimensional energy spectrum was calculated

$$
E_{\rho}(k)=\widehat{\rho}(k) \widehat{\rho}^{*}(k),
$$

where $\widehat{\rho}^{*}(k)$ is the complex conjugate of $\widehat{\rho}(k)$. To minimize errors associated with discontinuities between the beginning and end of the density trace, a Hann window was applied to the original trace of $\rho(x)^{\prime}$ (Blackman \& Tukey 1959).

Finally, the density data can be used to determine an integral measure of the degree of molecular mixing $\theta$ at a given downstream location. The measure used here was first introduced by Dankwerts (1952) as the degree of fluid segregation. The mathematical definition of $\theta$ requires the definition of two quantities, $B_{0}$ and $B_{2}$. The quantity

$$
B_{0}=\left(\frac{\rho_{r m s}}{\Delta \rho}\right)^{2}
$$

is the dimensionless variance of density fluctuations for miscible fluids at a given downstream location, where the root mean square $(\mathrm{rms})$ value of the fluctuating density trace is

$$
\rho_{r m s}=\sqrt{\lim _{L \rightarrow \infty} \frac{1}{L} \int_{0}^{L}\left[\rho(x)^{\prime}\right]^{2} d x} .
$$


The quantity

$$
\begin{aligned}
B_{2} & =\left\langle f_{1}\right\rangle\left\langle f_{2}\right\rangle \\
& =\left\langle f_{1}\right\rangle\left(1-\left\langle f_{1}\right\rangle\right) .
\end{aligned}
$$

is the dimensionless variance of density fluctuations for immiscible fluids at a given downstream location. Note that $\overline{f_{1}}$ is mathematically equivalent to $\left\langle f_{1}\right\rangle$ by Taylor's hypothesis, with the subtle distinction that $\bar{\phi}$ represents time-averaging and $\langle\phi\rangle$ represents spatial-averaging. Hereafter, spatial averaging will be performed assuming a space-time transformation by Taylor's hypothesis. Then the molecular mixing fraction at a given downstream location is defined as

$$
\theta=1-\frac{B_{0}}{B_{2}}
$$

By definition $\theta=0$ when the two fluids are completely segregated and $\theta=1$ when they are completely mixed. All quantities, $B_{0}, B_{2}$, and $\theta$ are dependent upon the distance downstream or $\tau$; however, that dependence has been is not shown in the above definitions for convenience.

\subsection{PIV Diagnostics}

Velocity perturbations in the streamwise direction were measured using particle image velocimetry (PIV) (Adrian 1991). The PIV system consisted of two $120 \mathrm{~mJ}$ ND-Yag lasers $(532 \mathrm{~nm})$, each with a firing rate of $15 \mathrm{~Hz}$ and a pulse width of 5 ns. The lasers are triggered in an alternating fashion, resulting in an effective $30 \mathrm{~Hz}$ sampling rate. Each 500-gallon water tank is seeded with $7.5 \mathrm{ml}$ of neutral-buoyancy silver spheres having a mean particle diameter of $10 \mu \mathrm{m}$. A series of cylindrical lenses created a sheet of laser light in the $x z$-plane of the water channel, as seen in figure 
2.1 and 2.3. The laser sheet was positioned so that the $u$ and $w$ components of the velocity field were measured in a plane just off the splitter plate. The measurements of $u$ and $w$ are then decomposed into their respective mean $(\bar{u}$ and $\bar{w})$ and fluctuating $\left(u^{\prime}\right.$ and $\left.w^{\prime}\right)$ components, as shown in equation (1.2).

Images were captured using a Kodak Megaplus ${ }^{\mathrm{TM}}$ digital imaging system. The image capturing system and laser triggering system were synchronized through the use of a pulse generator (Kraft 2004). A series of 1200 images of domain size $3.80 \mathrm{~cm}$ $\times 2.85 \mathrm{~cm}$ and a resolution of $640 \times 480$ pixels were recorded using $L a b V I E W^{\mathrm{TM}}$. Instantaneous velocity fields were determined by calculating the two-dimensional crosscorrelation of two successive images using MATPIV (Grue et al. 2000; Sveen 2004). Particle displacement vectors were calculated from the cross correlation of an interrogation window within two successive images:

$$
R(x, y)=\sum_{i=-M / 2, j=-N / 2}^{M / 2, N / 2} \operatorname{Im}_{1}(i, j) \operatorname{Im}_{2}(i+x, j+y)
$$

where $I m_{1}$ and $I m_{2}$ are the two-dimensional, 8-bit scalar intensity fields recorded by the image capturing system. The particle displacement vector is defined as the distance between the center of the interrogation window and the peak of the crosscorrelation function in two-dimensional space. Displacement vectors outside two standard deviations from their neighbors were discarded and replaced with interpolated values.

The MATPIV post-processing algorithm employed a multi-pass technique, where the initial pass operated on $64 \times 64$ pixel interrogation windows. The second pass used the displacement vectors from the first pass as initial estimates for $32 \times 32$ pixel interrogation windows. In both the first and second pass, the interrogation windows overlapped by $50 \%$, resulting in a final field of $39 \times 29$ velocity vectors. To 


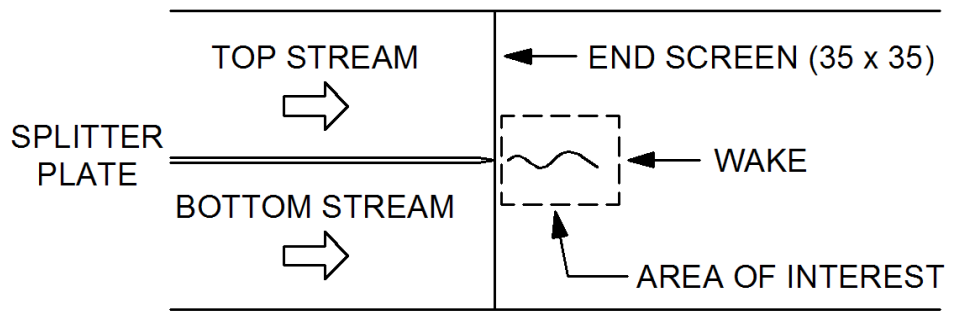

Figure 2.3 Schematic of the domain measured by the PIV system in the water channel.

examine the fluctuations of the velocity field at a given point in space, the $u$ and $w$ velocity components at a given $(x, y)$ location were taken from each of the 1199 velocity fields. Then, the velocity traces $u(t)$ and $w(t)$ were filtered using a SavitzkyGolay high-order polynomial filtering algorithm (9th-order over 19 points) to remove spurious velocity measurements (Ramaprabhu 2003). The uncertainty of the velocity measurements was determined by Ramaprabhu (2003) to be $0.05 \mathrm{~cm} / \mathrm{s}$ using methods described by Adrian (1997). Also, criteria for the statistical convergence of fluctuating velocity statistics were also presented by Ramaprabhu (2003) and, therefore, will not be presented here.

To quantify the initial velocity perturbations at the two-fluid interface, PIV measurements were performed in the wake immediately following the trailing edge of the splitter plate and end-screen $(0-2.5 \mathrm{~cm})$. Velocity measurements were taken with no temperature difference between the two streams. The removal of buoyancy-generated dynamics from the flow served two purposes. First, an objective of this investigation is to determine the momentum disturbance generated by the experimental apparatus, which excludes velocity fluctuations due to buoyancy effects. In the variable-density case with $A>0$, which constitutes the Rayleigh-Taylor unstable case, the momentum of oscillating structures (up-swells and down-swells) are different due to the difference in densities; however, in the limit of $A \rightarrow 0$, this difference is negligible and the 
current procedure is valid.

Similar to the density measurements, the determination of the range and magnitude of fluctuating velocities are of primary interest in these measurements. Equations (2.8) and (2.11) are used in a similar manner to calculate $\widehat{w}(k)$ and $E_{w}(k)$. The energy spectrum of the vertical velocity component, $E_{w}(k)$, at $x=0.5$ was used to parameterize the initial velocity perturbation of the flow, which will be discussed in $\S 4.3$. Also of interest was the vertical variation in the $u$ velocity profile. This profile will also be used in the parameterization of the initial velocity conditions in $§ 4.3$.

\subsection{PLIF Diagnostics}

Planar laser-induced fluorescence (PLIF) techniques were used to measure the interfacial perturbation in the spanwise $(y)$ direction. PLIF was used to detect the presence of a particular scalar by injecting into (or seeding) a fluid stream with a dye that fluoresces when excited by a given wavelength of light (Jacobs 1992; Lee et al. 1992). To accomplish this, the current optical arrangement employed by the PIV system was modified so that the laser sheet was perpendicular to the mean channel flow. A schematic of the camera and laser sheet arrangement is shown in figure 2.4. Rhodamine $6 \mathrm{G}$ dye $(2.5 \mathrm{~g})$ was added to the 500 -gallon tank containing the top (cold) stream so that it would fluoresce in the presence of $532 \mathrm{~nm}$ laser light. The laser sheet was placed a distance $x=0.5 \mathrm{~cm}$ from the trailing edge of the splitter plate so that only the top stream was illuminated, thus allowing the determination of the two-fluid interfacial perturbation. The digital imaging system used in the thermocouple and PIV experiments was modified to record images of $1000 \times 480$ pixels over a domain size of approximately $10 \mathrm{~cm} \times 5 \mathrm{~cm}$. The imaging system was synchronized with the laser system in the same manner as described in $\S 2.3$. 


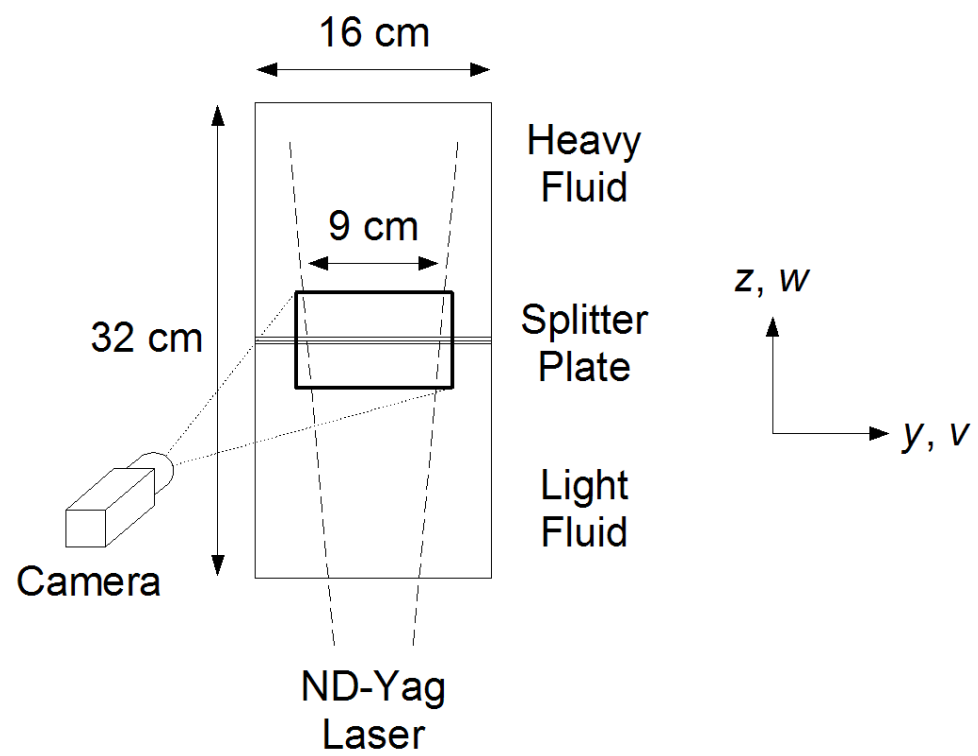

Figure 2.4 Schematic of the experimental laser sheet configuration for spanwise PLIF measurements.

These spanwise measurements are novel in the following way. These are the first experiments to employ off-axis imaging of the water channel, requiring new experimental procedures to be developed for this experimental configuration. More importantly, these are the first measurements of the initial seeding in the spanwise direction of a canonical Rayleigh-Taylor flow. Combined with the measurements described in $\S 2.2$ and $\S 2.3$, these measurements provide the most complete description of the initial conditions of a Rayleigh-Taylor flow to date.

Spanwise interfacial measurements were performed as follows. First, the laser head and all associated optics were configured such that a vertical laser sheet was formed in a plane $x=0.5 \mathrm{~cm}$ downstream from the splitter plate. The camera was located approximately $50 \mathrm{~cm}$ from the midpoint of the trailing edge of the splitter plate, and the focal axis was located on the same horizontal plane as the splitter plate at an angle approximately $35^{\circ}$ from the axis normal to the laser sheet. The 
laser sheet was focused so that its thickness was $<1 \mathrm{~mm}$ near the centerplane of the channel. Using a syringe, a solution of Rhodamine 6G dye and water was injected into the channel so that the camera could be focused. Once the camera was focused, the water channel pumps were activated and a series of images were captured. For the same reasons discussed in $\S 2.3$, the water channel was operated with no temperature difference between the two streams. Measurements were also performed with $A=$ $5.0 \times 10^{-4}$, with no difference found between the measurements performed with $A=0$. Thus, the measured perturbations do reflect the interfacial perturbation imposed by the splitter plate and end-screen on the two-fluid interface in the spanwise direction.

Once images of the two fluid interface had been captured, the water channel was drained and a calibration grid was placed in the same location as the laser sheet. The calibration grid consisted of an array of horizontal and vertical lines located at half-inch intervals, and was used to eliminate any parallax and perspective errors introduced by locating the camera at an off-angle to the laser sheet. The calibration grid was mounted between two sheets of clear Plexiglas $0.64 \mathrm{~cm}$ in thickness to ensure that the grid remained flat during the course of the experiment. Once the calibration grid was in place, an image of the grid was recorded with the same camera settings and location as used to capture the PLIF images. Images of the calibration grid were used to calibrate the dewarping procedure described below.

Special consideration has been given to the dewarping of the spanwise images. Other researchers have used off-axis imaging and dewarping algorithms in applications such as stereoscopic PIV (Arroyo \& Greated 1991; Willert 1997) and other applications (Kent \& Eaton 1982). However, in these experiments, the location of the water channel and laser sheet determined the location of the camera and not the need to resolve an out-of-plane velocity component. The procedure used in this investigation is the same robust, second-order dewarping procedure presented by Pratt (1991) 
and van Oord (1997). The functional relationship between the warped coordinates $\left(X_{w}, Y_{w}\right)$ and dewarped coordinates $\left(X_{d}, Y_{d}\right)$ is given by

$$
\begin{gathered}
X_{d}\left(X_{w}, Y_{w}\right)=a_{0}+a_{1} X_{w}+a_{2} Y_{w}+a_{3} X_{w} Y_{w}+a_{4} X_{w}^{2}+a_{5} Y_{w}^{2} \\
Y_{d}\left(X_{w}, Y_{w}\right)=b_{0}+b_{1} X_{w}+b_{2} Y_{w}+b_{4} X_{w} Y_{w}+b_{4} X_{w}^{2}+b_{5} Y_{w}^{2}
\end{gathered}
$$

where $a_{i}$ and $b_{i}, i=1-5$, are calibrated coefficients. The unknown coefficients were determined from a least-squares fit relating 15 control points from the warped image of the calibration grid to their true physical location. Figure 2.5 shows the raw warped image of the calibration grid and a corrected image.

Once a PLIF image has been corrected for perspective errors, the two-fluid interface can be extracted by analyzing the intensity values at each pixel location of the PLIF image. A Canny-type edge filter was used to determine the interface between the fluorescing and non-fluorescing fluids (Canny 1986). The Canny method identifies edges in the image at local maxima of the gradient of the intensity field, given a threshold value to eliminate spurious boundaries. Results using this method will be presented in $\S 3.3$.

An issue with the use of PLIF diagnostics in the water channel is that the diffusivity of heat and the diffusivity of the fluorescing dye marker are very different. A temperature difference between the two streams is used to create the density difference: thus, the two-fluid mixture that is created by this arrangement is miscible, such that the Prandtl number is $\operatorname{Pr} \equiv \nu / D \approx 7$, where $\nu$ is the kinematic viscosity and $D$ is the thermal diffusivity for water. However, the dye dissolved in the top stream diffuses into the bottom stream at a much slower rate than the diffusion of heat. Thus, the question is raised whether or not the dye is an accurate marker for the two-fluid interface. However, this error is minimized in this investigation by 

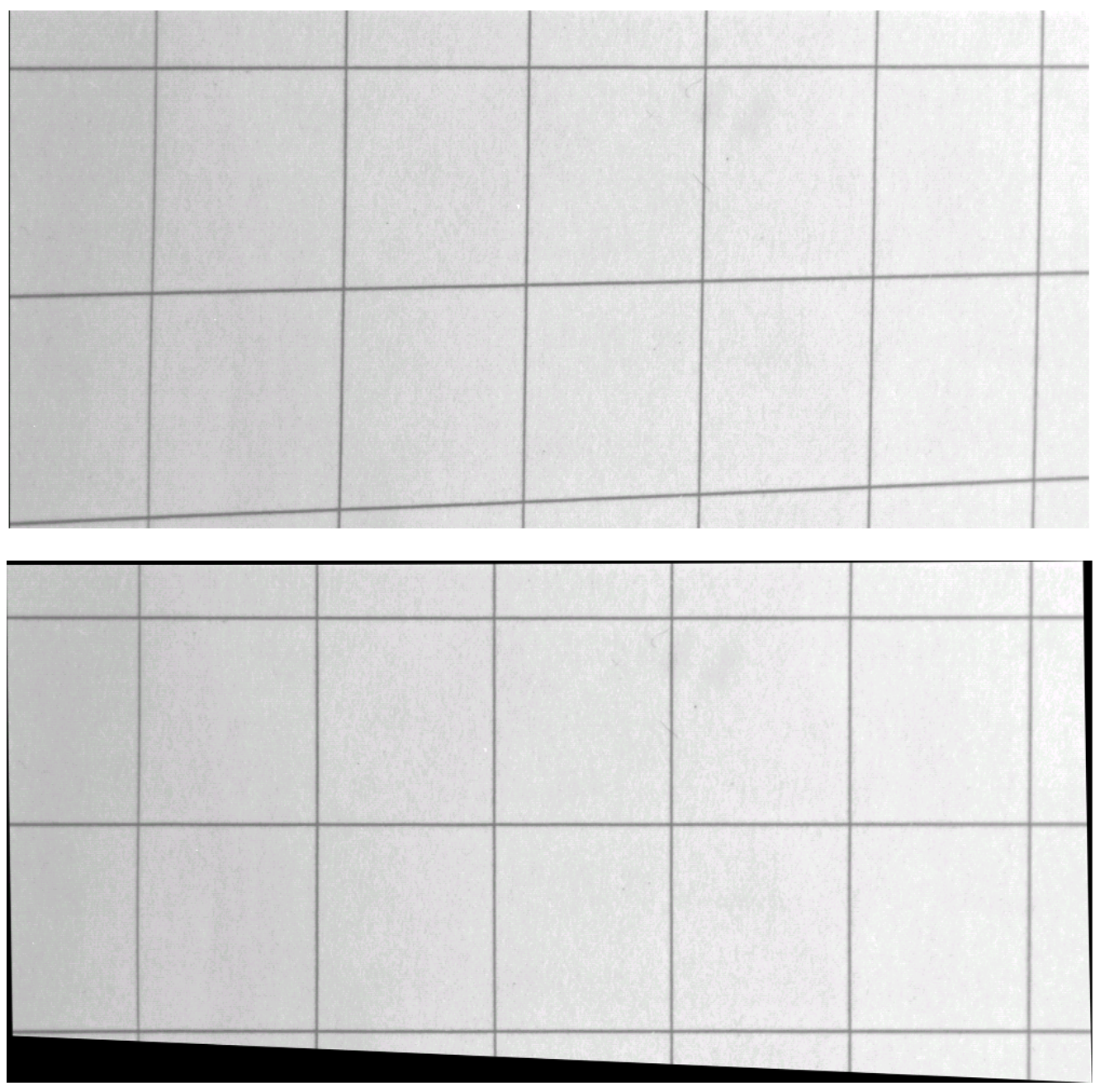

Figure 2.5 Raw image of warped calibration grid (above) and dewarped calibration grid (below). 
taking measurements in the limit of $\tau \rightarrow 0$, such that the difference between the amounts of diffusion is negligible. Aside from the difference being negligible at very early times, the location of the two-fluid interface is the only desired information from the measurement, rather than data concerning the internal structure, also justifying the current procedure. 


\section{EXPERIMENTAL RESULTS}

\subsection{Density Measurements}

Density measurements were taken at several downstream locations, $x=0.1,1.0$, 5.0, 10.0, 20.0, 30.0, and $40.0 \mathrm{~cm}$. Of particular interest are the two measurements closest to the splitter plate because of their potential to represent the initial interfacial perturbation of the flow. Figure 3.1 shows the evolution of the volume fraction probability density function (PDF) $P\left(f_{1}\right)$ for several downstream locations. All curves shown in figure 3.1 were measured at the centerline, showing that while $\left\langle f_{i}\right\rangle=1 / 2$, much more information concerning the internal structure and degree of molecular mixing is present in the trace of the density values than just the average volume fraction. The early-time $(\tau=0.004$ and $\tau=0.04)$ volume fraction distributions are peaked at $\left\langle f_{i}\right\rangle=1 / 2$, indicating that the majority of the fluid at the centerline is mixed. However, at intermediate times $(0.19<\tau<0.39)$, the distribution becomes bimodal. This trend is reversed at later times, as the distribution of $\left\langle f_{i}\right\rangle$ returns to a single-mode distribution in the large Reynolds number regime.

Similar volume fraction distributions were reported by Ramaprabhu and Andrews (2004a) using lower resolution thermocouples; however, the degree of kurtosis or bimodality differed from the distributions presented in this investigation. This is attributed to the inability of the larger thermocouples used by Ramaprabhu and Andrews to capture the sharp gradients present in the early stages of mixing. Further information on the spatial-averaging due to thermocouple probe size can be found in Appendix A.

As shown in figure 3.1, the amount of mixed and unmixed fluid present at the centerplane of the mixing layer varies significantly with downstream position. Another illustration of this is shown in the evolution of the molecular mixing fraction $\theta$, shown 

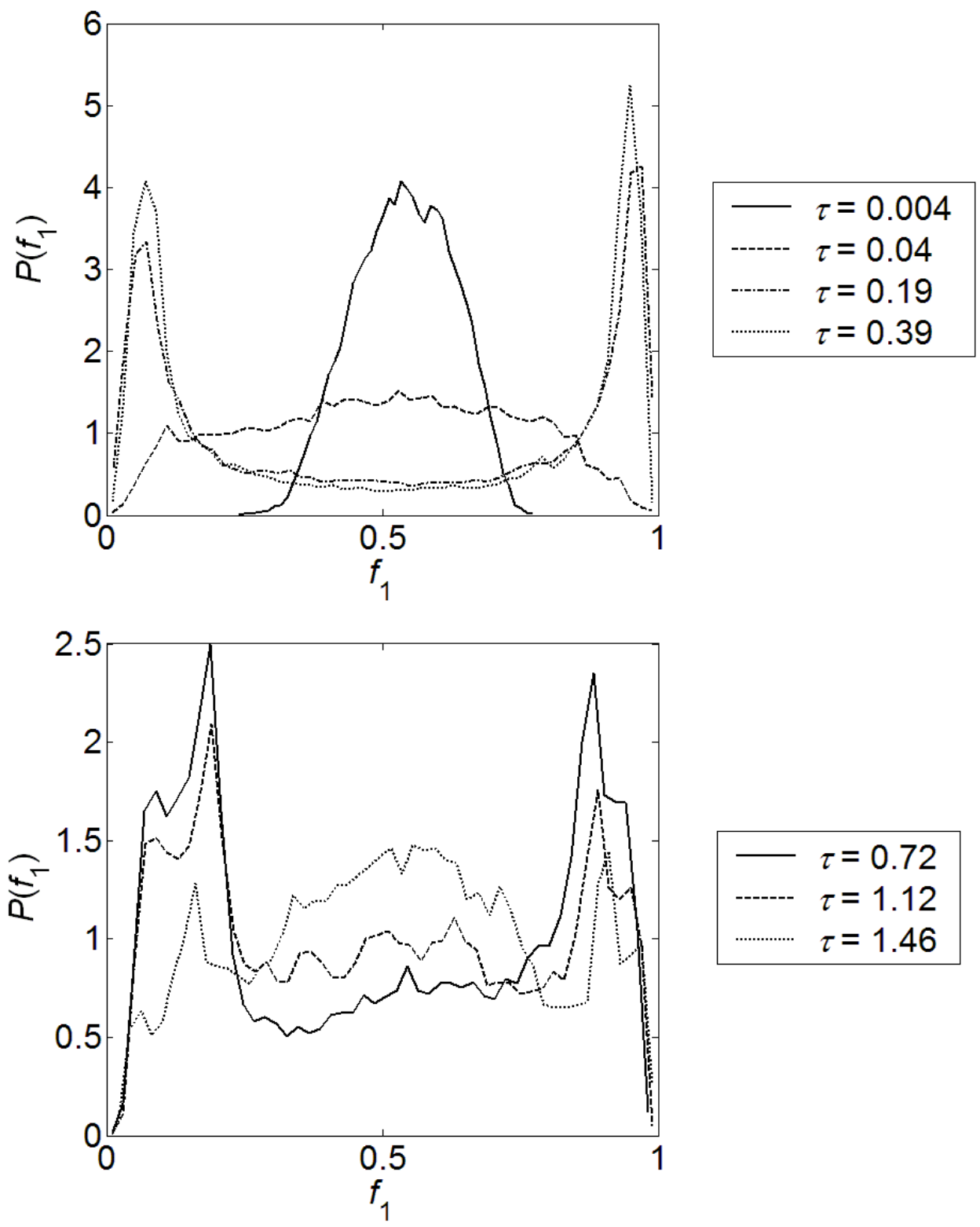

Figure 3.1 Time-evolution of early-time (top) and late-time (bottom) volume fraction PDF, $P\left(f_{1}\right)$. 
in figure 3.2. There are two stages of evolution which are noteworthy in figures 3.1 and 3.2. At very early times, the value of $\theta$ decreases rapidly, which represents the transition from a perturbed two-fluid interface to the initial formation of a mixing layer in the linear and weakly-nonlinear regimes. The second stage is noted by the rise in value of $\theta$. Once the growth rates of the initial perturbations saturate and strongly-nonlinear dynamics control the growth of the mixing layer, the interfacial area between the two fluids increases with the onset of Kelvin-Helmholtz instabilities, thereby enhancing the mixing process. This transition is observed in the water channel experiments at $\tau \approx 0.3$, which is shown in the minimum value of $\theta$.

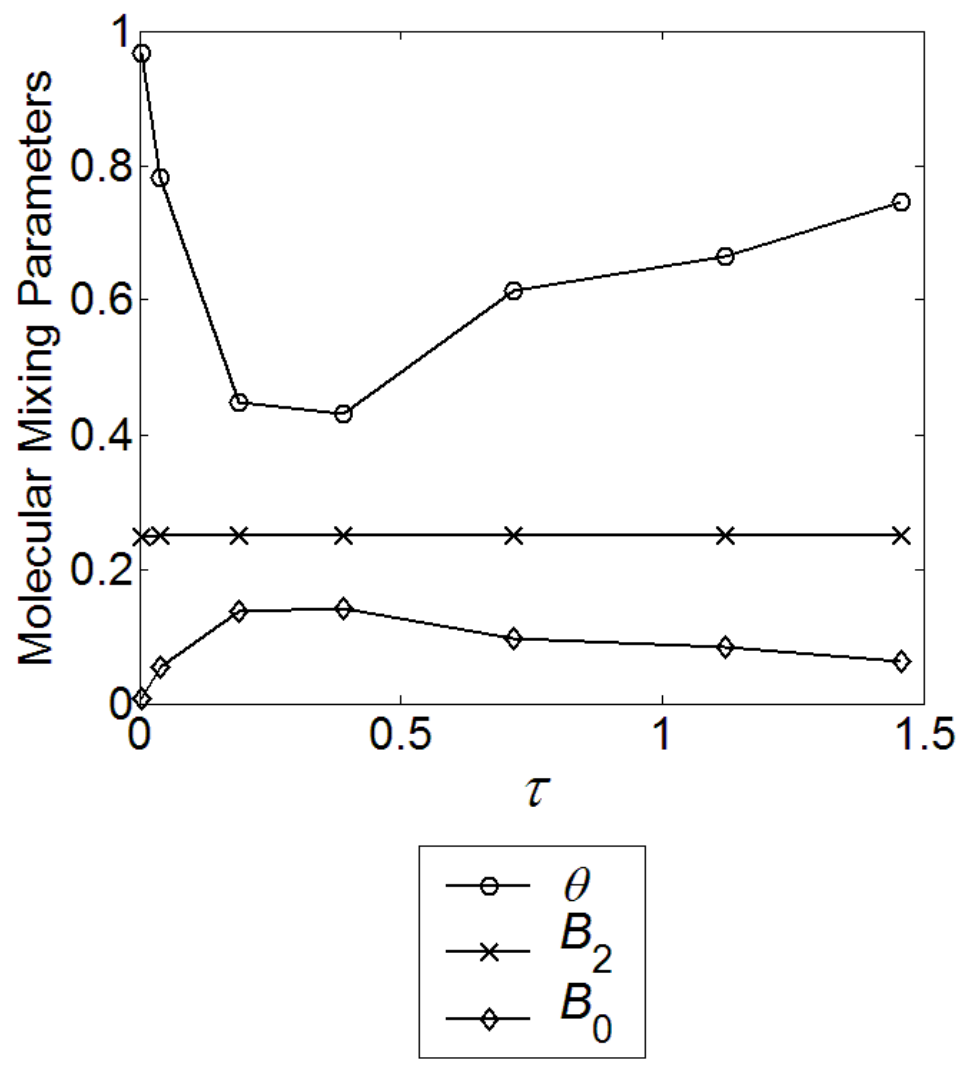

Figure 3.2 Time-evolution of the molecular mixing quantities, $B_{0}, B_{2}$ and $\theta$. 
Molecular mixing between two fluids in a Rayleigh-Taylor mixing layer is difficult to measure experimentally because of the requirement for sufficient data points to calculate $\rho_{r m s}$ and $\left\langle f_{1}\right\rangle$ on a particular $z$-plane, with the constraint that the measurement points are finely spaced so that density gradients can be resolved. Such requirements are more easily achieved in numerical simulations. Historically, researchers have typically reported values of $\theta(z)$ across the mixing layer for various $\tau$, rather than the evolution of $\theta(z=0, \tau)$, as shown here. Ramaprabhu and Andrews (2004a) measured $\theta(z=0, \tau)$ in the same water channel experiment used in this investigation; however, a discrepancy exists in the values of $\theta$ measured between $0<\tau<1$. Ramaprabhu and Andrews reported a minimum value of $\theta \approx 0.68$ at $\tau \approx 0.3$, whereas a minimum of $\theta \approx 0.45$ is reported here. The differences between the measured values of $\theta$ reported here and by Ramaprabhu and Andrews are attributed to the difference in the size of the thermocouple probes used and the noise elimination techniques employed (see Appendices A and B). Ristorcelli and Clark (2004) used DNS with $S c=1$ and isotropic interfacial perturbations to measure the evolution of $\theta$ at the centerplane of the mixing layer. Ristorcelli and Clark hypothesized that for a self-similar state to exist, $\theta$ must reach an asymptotic value beyond the transition to the turbulent, self-similar regime. While many of the numerically-measured turbulence and mixing statistics, such as $\left\langle w^{\prime 2}\right\rangle,\left\langle\rho^{\prime} w^{\prime}\right\rangle$ and $\left\langle\rho^{\prime 2}\right\rangle$, collapsed onto apparently self-similar profiles, an asymptotic value of $\theta$ was never attained. Ristorcelli and Clark (2004) showed similar qualitative results to those shown here despite a significant difference in initial conditions of the flow, where $\theta$ initially decreased to some minimum value in the early stages of development, followed by an increase towards late-time values of $\theta \approx 0.8$.

The time-evolution of the volume fraction PDF (figure 3.1) and molecular mixing fraction (figure 3.2) indicate the same imbalance between the rates of fluid engulfment 
and molecular mixing. The term "engulfment" is intended in this case because term "entrainment" is typically used in shear layer applications where the physical mechanism by which fluid enters the mixing layer is different than that of a Rayleigh-Taylor driven mixing layer. First, a transition exists at a given time early in the development of the mixing layer where the rates of fluid engulfment and molecular mixing are matched. Before the onset of strong nonlinearities, the growth of the initial buoyant structures engulfs unmixed fluids into the mixing layer. Beyond this transition, the rate of molecular mixing due to turbulent mixing is greater than the fluid engulfment rate. As $\tau$ increases, the ratio of the engulfment and molecular mixing rates shows the tendency towards reaching some degree of balance. Dimotakis (2000) proposed that a point exists in a turbulent flow, where above the limit of $R e \equiv L U / \nu \approx 10^{4}$ or $R e_{\lambda} \equiv \lambda u^{\prime} / \nu \approx 100$, turbulent motions increase the rate of molecular mixing as opposed to turbulent straining of the two-fluid interface, where $R e$ is a large-scale Reynolds number, $L$ and $U$ are selected length and velocity scales, $R e_{\lambda}$ is the TaylorReynolds number, and $\lambda$ is the Taylor microscale (Pope 2000). Previous researchers have measured the Taylor-Reynolds number in the water channel at $\tau=1.21$ to be $R e_{\lambda} \approx 60$ (Ramaprabhu \& Andrews 2004a). Thus, these experimental measurements fall short of the proposed Taylor-Reynolds limit set by Dimotakis. Consequently, it is unclear whether an asymptotic value of $\theta$ is achievable or if the mixing transition can occur in this flow, based upon these criteria.

Also of interest is the range of scales present in the fluctuating density field. Figures 3.3-3.6 show the time-evolution of the density power spectra, which have not been normalized so that they may be directly parameterized and implemented in numerical simulations of the experiment. These topics will be addressed in Sections 4 and 5.

The early-time spectra $(\tau=0.004$ and $\tau=0.04)$ shown in figure 3.3 exhibit a 

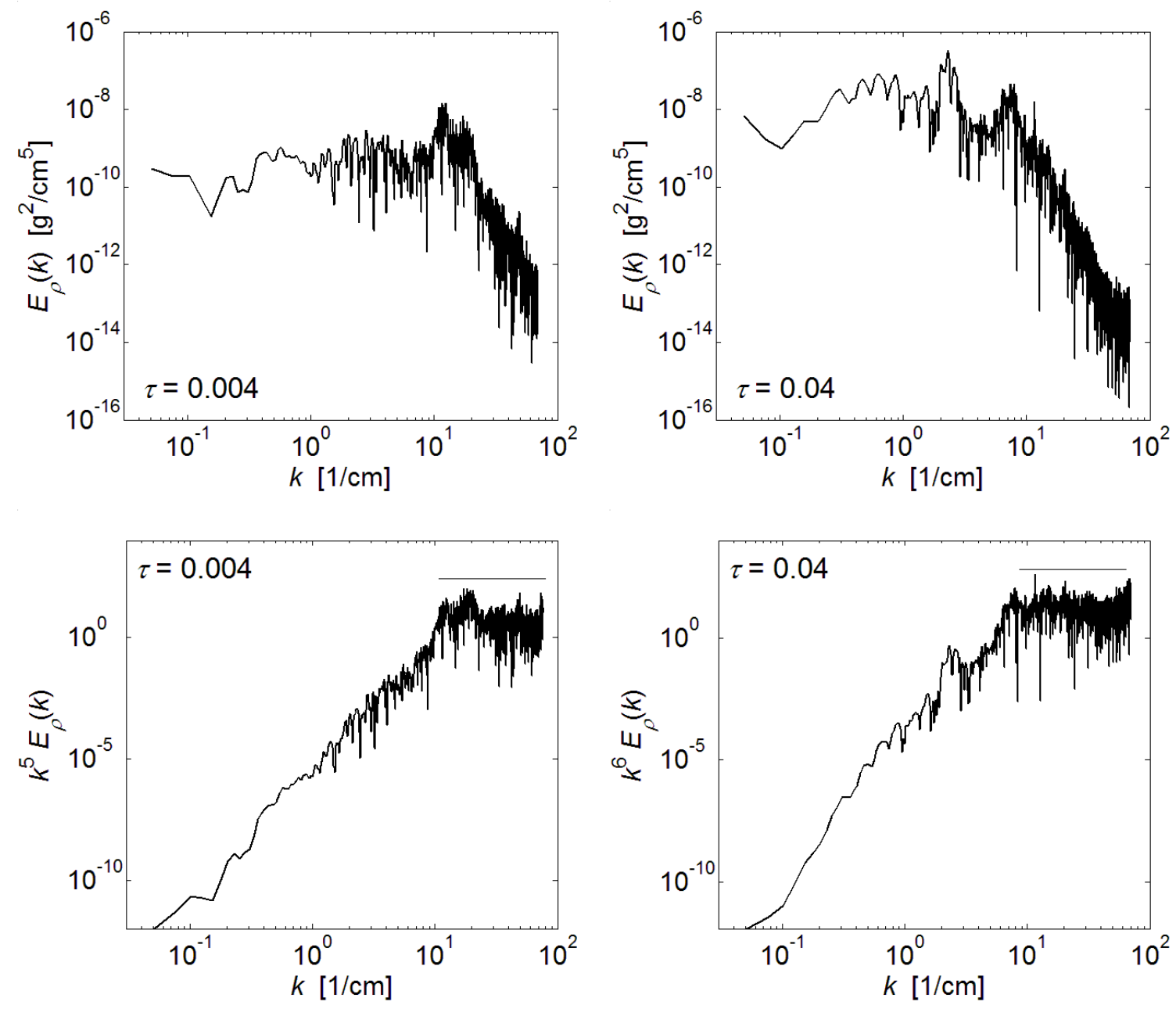

Figure 3.3 Density power spectrum for $\tau=0.004$ (above left) and $\tau=0.04$ (above right). The compensated spectrum for $\tau=0.004$ exhibits a power-law of $k^{-5}$ (below left) which differs slightly from the power-law of $k^{-6}$ shown at $\tau=0.04$. 

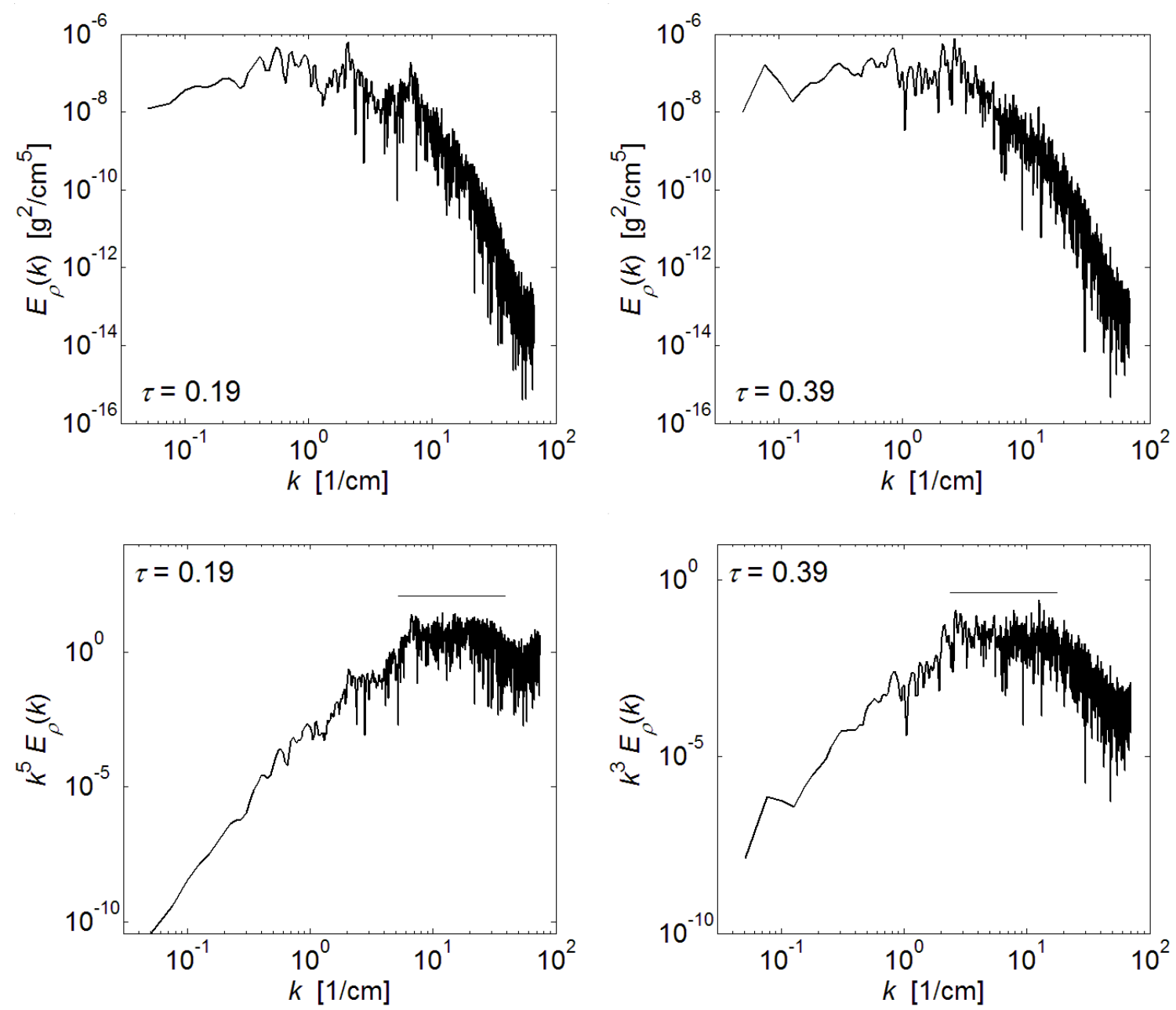

Figure 3.4 Density power spectrum for $\tau=0.19$ (above left) and $\tau=0.39$ (above right). The compensated spectrum for $\tau=0.19$ exhibits a power-law of $k^{-5}$ (below left) which differs from the power-law of $k^{-3}$ shown at $\tau=0.39$ (below right). 

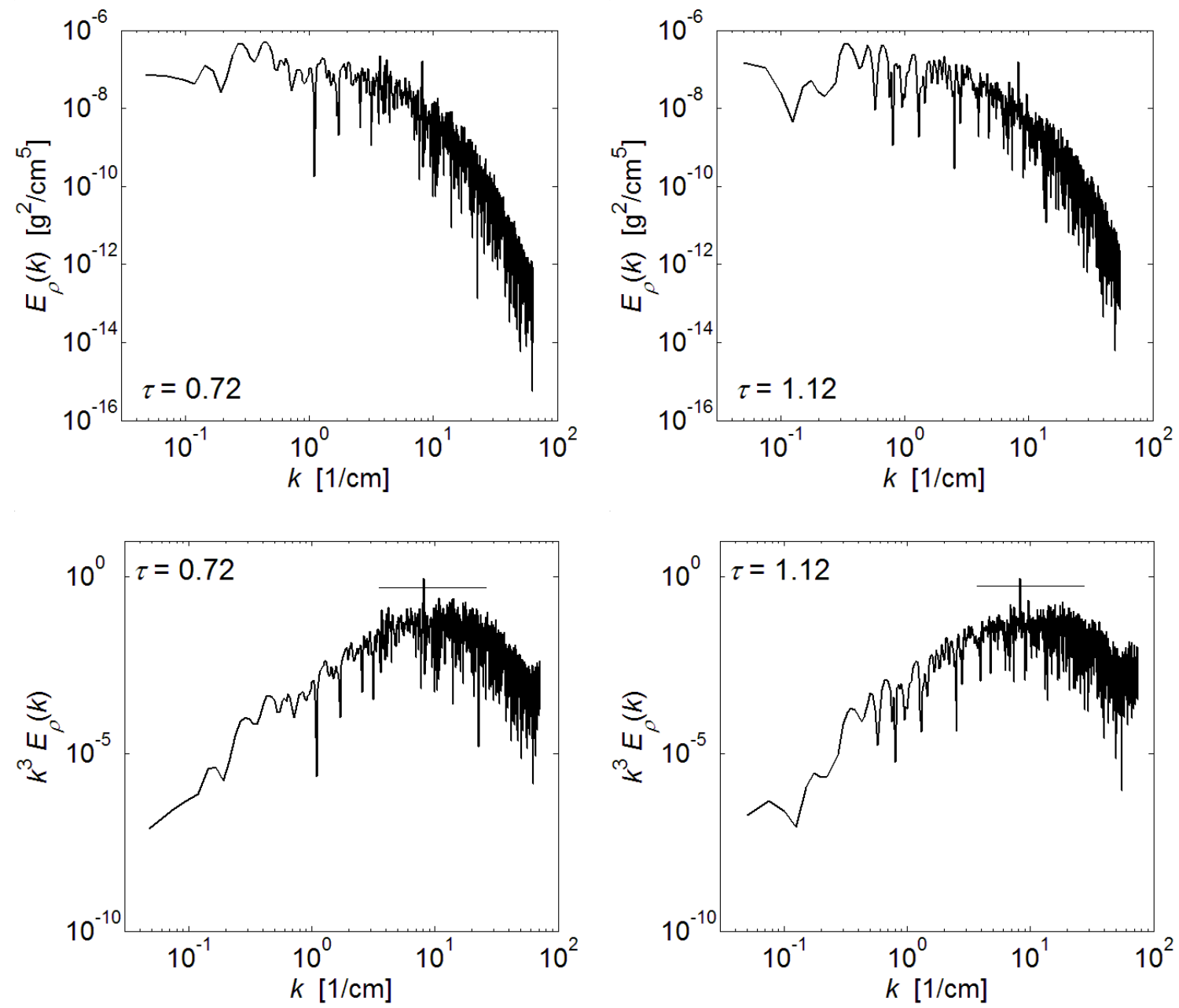

Figure 3.5 Density power spectrum for $\tau=0.72$ (above left) and $\tau=1.12$ (above right). The compensated spectrum for $\tau=0.72$ (below left) and $\tau=1.12$ (below right) exhibit an apparent power-law of $k^{-3}$. 

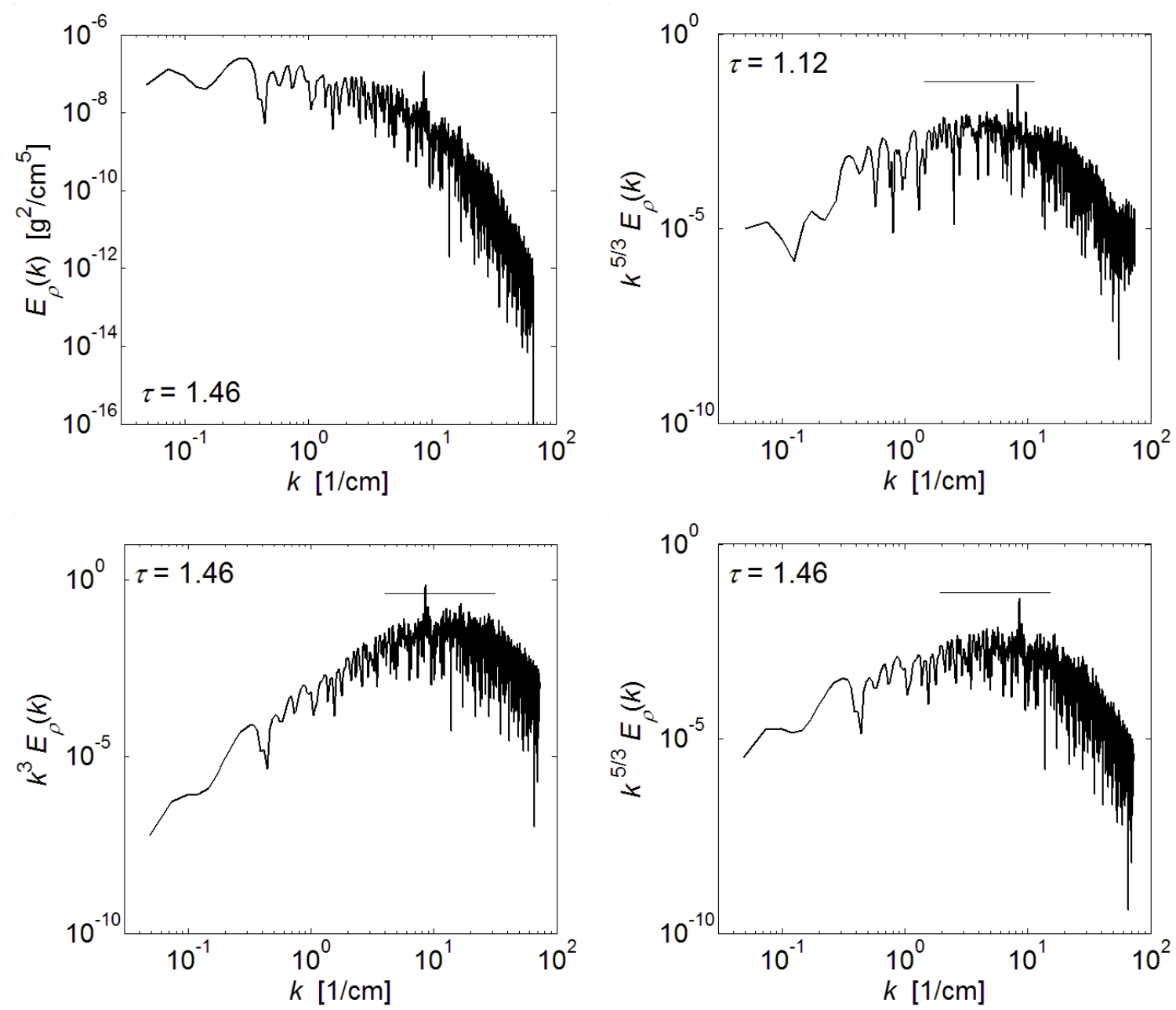

Figure 3.6 Density power spectrum for $\tau=1.46$ (above left) and $k^{-3}$ compensated spectrum (below right). The $k^{-5 / 3}$ compensated spectra for $\tau=1.12$ (above right) and $\tau=1.46$ (below right) exhibit less than one decade of modes that apparently scale as $E_{\rho}(k) \sim k^{-5 / 3}$. 
broad-band of perturbations that exist at the onset of the instability; however, the density power spectrum at $\tau=0.04$ has considerably more energy in the wavenumber range of $0.1<k<4$. This is due to the fact that the initial perturbations in the streamwise direction are driven by velocity fluctuations. At $\tau=0.004$, or $x=0.1 \mathrm{~mm}$ from the trailing edge of the splitter plate, the velocity perturbations have not had sufficient time to deform the interface, resulting in the difference in energy content between $0.1<k<4$. The peak value of $E_{\rho}(k)$ at $k \approx 10$ for both $\tau=0.004$ and $\tau=0.04$ is believed to be imposed by the combination of the splitter plate and endscreen, and not by velocity perturbations. A qualitative relationship between the initial density and velocity spectra will be presented in the next section. From linear stability analysis of a Rayleigh-Taylor unstable interface including viscous effects, the most unstable wavelength may be determined from (Chandrasekhar 1961):

$$
\lambda_{m} \approx 4 \pi\left(\nu^{2} A g\right)^{1 / 3}
$$

Assuming that $\nu \approx\left(\mu_{1}+\mu_{2}\right) /\left(\rho_{1}+\rho_{2}\right)$ and $A=7.5 \times 10^{-4}$, the most unstable wavelength is $\lambda_{m}=0.53 \mathrm{~cm}$ or $k \approx 12 \mathrm{~cm}^{-1}$. The peak density perturbation of the density spectra at $\tau=0.004(x=0.1 \mathrm{~cm})$ approximately matches the most unstable wavelength with a peak at $k=11 \mathrm{~cm}^{-1}$. The relationship between the initial density spectrum and the spectrum of vertical velocity fluctuations near the edge of the splitter plate will be discussed further in $\S 3.2$.

While the early-time $(\tau=0.004$ and $\tau=0.04)$ spectra exhibit broad-banded energy content, the peak perturbations occur in the intermediate-wavenumber range, $1<k<10$. For both cases, the peak perturbations have energy content that is two orders of magnitude greater than the long-wavelength content of the spectrum. Relating the energy content to the amplitude of the perturbation, $|\widehat{\rho}(k)|=\sqrt{E_{\rho}(k)}$, yields a ratio of peak perturbation amplitude to long-wavelength perturbation amplitude of 
$\widehat{\rho}_{\text {peak }} / \widehat{\rho}_{k<1} \approx 10$.

As the mixing layer develops, an apparent short-wavelength scaling of $k^{-3}$ can be seen in the intermediate wavenumbers for $\tau=0.19$ and $\tau=0.39$, as shown in figure 3.4. While no formal theory has been developed explaining such a scaling, similar results have been reported by researchers investigating turbulent buoyant plumes (Papanicolaou \& List 1987; Dai et al. 1994; Fisher \& Ball 1999) and also by previous researchers using the water channel to study Rayleigh-Taylor mixing (Wilson \& Andrews 2002; Ramaprabhu \& Andrews 2004a). Wilson and Andrews hypothesize that an inertial-diffusive range exists where concentration (or density) fluctuations persist to higher wavenumbers than do velocity fluctuations, because the thermal diffusivity is almost an order of magnitude less than the kinematic viscosity in the water channel.

At later times, $\tau=1.12$ and $\tau=1.46$, an apparent short inertial range scaling of $k^{-5 / 3}$ is formed, where the $k^{-5 / 3}$ compensated spectra shown in figure 3.6 occupies less than one decade of wavenumbers. Wilson and Andrews (2002) and Ramaprabhu and Andrews (2004a) reported similar findings at these downstream positions using larger thermocouples. Ramaprabhu and Andrews have shown that at $\tau=2.08$, an inertial range for $E_{\rho}(k)$ exists over approximately one decade of wavenumbers. Ramaprabhu and Andrews also estimated the integral-scale Reynolds number at $\tau=$ 1.21 to be $R e \equiv L U / \nu=1000-1450$, depending upon the choice of lengthscale $L$ and velocity-scale $U$ used, indicating the minimum Reynolds number required for the initial formation of an inertial range for the Atwood and Schmidt numbers of the experiment. 


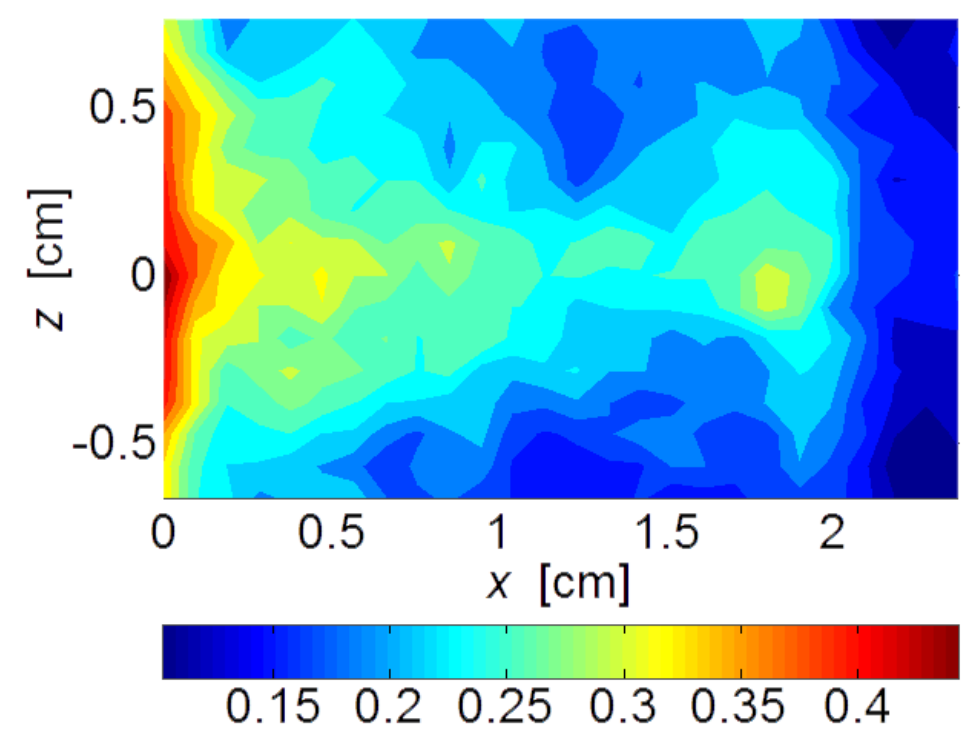

Figure 3.7 Field of $w_{r m s}$ at downstream locations $x=0-2.5 \mathrm{~cm}$.

\subsection{Velocity Measurements}

Velocity measurements were taken in the $x z$-plane so that the initial velocity fluctuations present at the trailing edge of the splitter plate could be quantified. A contour plot of $w_{r m s}$ is shown in figure 3.7, where the wake behind the splitter plate is clearly visible. The peak $w_{r m s}$ values occurring to the left of the image are residual noise from the laser light reflected from the steel end-screen and do not represent physical values. An accurate measurement of $w_{r m s}$ in the limit of $x \rightarrow 0 \mathrm{~cm}$ is not feasible due to this noise. However, it is possible to examine the decay of $w_{r m s}$ along the centerline of the flow so that $w_{r m s}(x=0)$ may be estimated. Figure 3.8 shows the spatial-evolution of $w_{r m s}$ on a logarithmic scale, showing that $w_{r m s} \sim x^{-1 / 2}$ as $x \rightarrow 0$. This relationship will be used later in $\S 4.3$ to estimate the total kinetic energy in the initial velocity spectrum at $\tau=0$. 


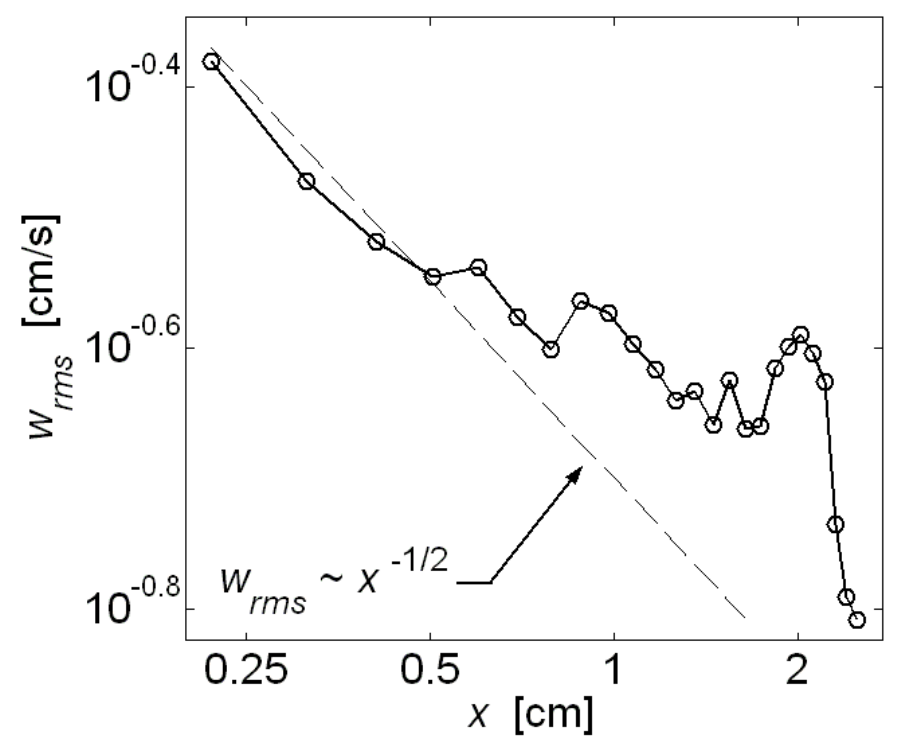

Figure 3.8 Early-time decay of $w_{r m s}$ in the streamwise direction following the trailing edge of the splitter plate with the channel in a non-buoyant configuration.

Similar to the measurement of the density fluctuations, the measurement of the initial vertical velocity spectrum $E_{w}(k)$ is crucial to the determination of the initial momentum perturbation on the two-fluid interface. Figure 3.9 shows the measured velocity spectrum at $x=0.5 \mathrm{~cm}$ downstream from the splitter plate. The total energy content at the centerline of the flow is determined by integrating the spectrum shown in figure 3.9 across all wavenumbers, where

$$
\begin{aligned}
E_{w}(x) & =\int_{k_{\min }}^{k_{\max }} E_{w}(k, x) d k \\
& =\left\langle w^{\prime 2}\right\rangle(x) \\
& =w_{r m s}^{2}(x) .
\end{aligned}
$$

Using the relationship that $w_{r m s}(x) \sim x^{-1 / 2}$, then $E_{w}(x) \sim x^{-1}$ and assuming negligible spectral transfer, it is possible to scale the measured spectrum $E_{w}(k, x=0.5)$ 
to estimate $E_{w}(k, x=0)$. The spectrum shown in figure 3.9 is also in its dimensional form so that it may be directly parameterized in a numerical simulation of the experiment.

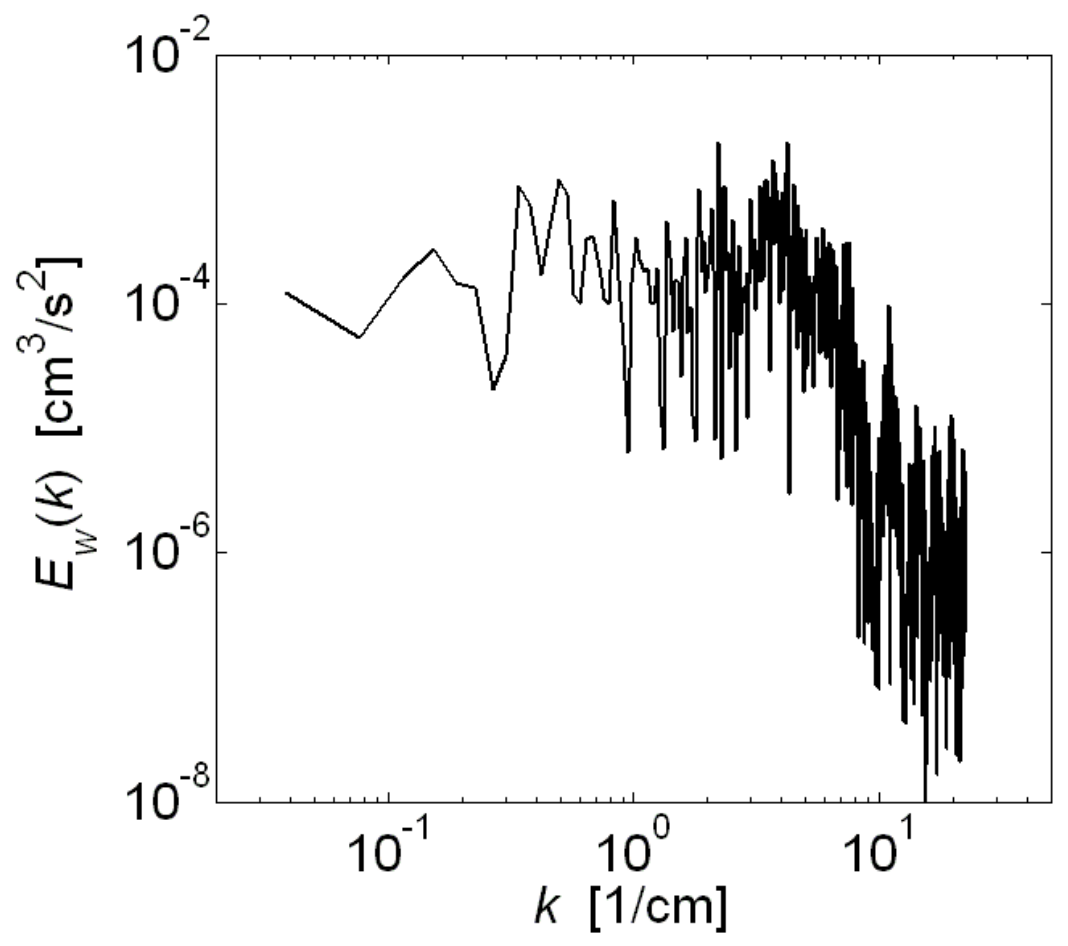

Figure 3.9 Spectrum of $w$ fluctuations at $x=0.5 \mathrm{~cm}$ from the splitter plate.

The velocity spectrum shown in figure 3.9 can be qualitatively related to the early-time density spectra shown in figure 3.3. The density spectrum at $\tau=0.04$ contains roughly three local maxima at $k \approx 0.8,2.5,9.0$. The peak value that occurs at $k=9.0$ does not correspond to a local maximum in the velocity spectrum in figure 3.9 , and therefore is believed to be a remnant of the splitter plate and end-screen. However, the maxima at $k=0.8$ and $k=2.5$ do correspond to local maxima in the vertical velocity spectrum at wavenumbers $k=0.7$ and $k=2.0$. Discrepancies between the exact peak wavenumber of $E_{\rho}(k)$ and $E_{w}(k)$ are attributed to experimental 
uncertainties. This qualitative correlation implies that the evolution of the density spectra at early times in the experiment are driven by the fluctuations in the velocity field and not by the deposition of baroclinic vorticity due to the Rayleigh-Taylor instability.

While vertical velocity fluctuations are believed to be the dominant mechanism contributing to the initial development of the Rayleigh-Taylor mixing layer in the water channel experiment, the current set of measurements allow for the quantification of some second-order effects at $\tau=0$. The same measurements for $E_{w}(k)$ allow the quantification of the velocity deficit in the wake trailing the splitter plate. Ramaprabhu (2003) reported a velocity deficit of approximately $10 \%$ of the mean advective velocity at $x=1.0 \mathrm{~cm}$ from the splitter plate. This deficit was negligible at $x=2.0 \mathrm{~cm}$, as buoyancy effects began to dominate the dynamics of the mixing layer. The current measurements exhibited a $5 \%$ difference between the mean advective velocity $\left(U_{m}=4.1 \mathrm{~cm} / \mathrm{s}\right)$ and the minimum velocity within the wake $\left(u_{\min }=3.9 \mathrm{~cm} / \mathrm{s}\right)$ at $x=0.5 \mathrm{~cm}$ downstream, as shown in figure 3.10 . This reduction in the velocity deficit behind the splitter plate is attributed to the existence of smaller boundary layers at the knife-edge of the splitter plate. The smaller boundary layers are a result of modifications to the upstream screen meshes, which is discussed in Appendix C.

\subsection{Spanwise Measurements}

Measurements in the spanwise direction were performed to quantify the initial interfacial perturbation at the interface between the cold and hot fluids. Once the interfacial perturbation in the spanwise direction $\zeta(y)$ has been measured, the corresponding energy spectrum $E_{\zeta}(k)$ can be computed and parameterized. Figure 3.12 shows the interfacial perturbation spectrum for one captured image, where other im- 


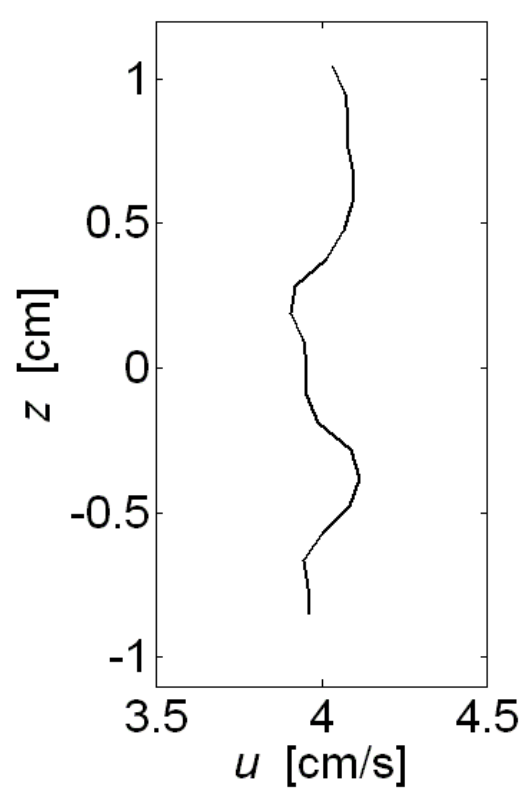

Figure 3.10 The variation of $\langle u(z)\rangle$ at $\tau=0.018$ for the experiment with the water channel in a non-buoyant configuration.

ages produced similar spectra. The spectrum shows a broad band of perturbations, with most of the energy content between intermediate wavenumbers or wavelengths $0.16<\lambda<5.0 \mathrm{~cm}$, well within the water channel width of $20 \mathrm{~cm}$. The maximum $5 \mathrm{~cm}$ wavelength is attributed to the maximum width of the laser sheet achievable at the two-fluid interface. A PLIF image of the spanwise interfacial perturbation is shown in figure 3.11 .

A key result of this experiment was the determination that velocity perturbations in the spanwise direction are negligible. This was confirmed by inspection of the fluorescing dye while the experiment was operating. If a spanwise velocity perturbation existed, the waveform created by the splitter plate and end-screen would oscillate in the $y$-direction. However, no such oscillation was observed, eliminating the need to 


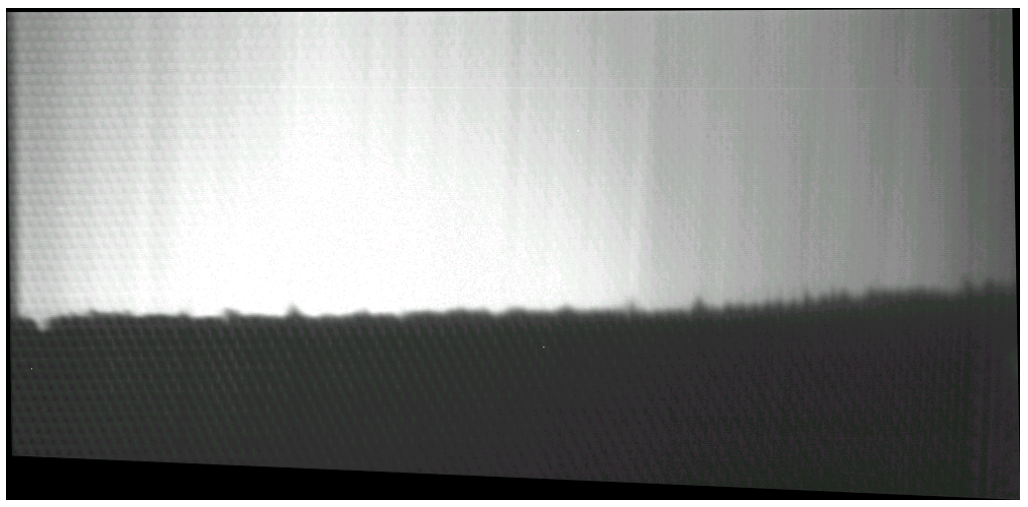

Figure 3.11 PLIF image of spanwise interfacial perturbation.

perform velocity measurements in the $y z$-plane. 


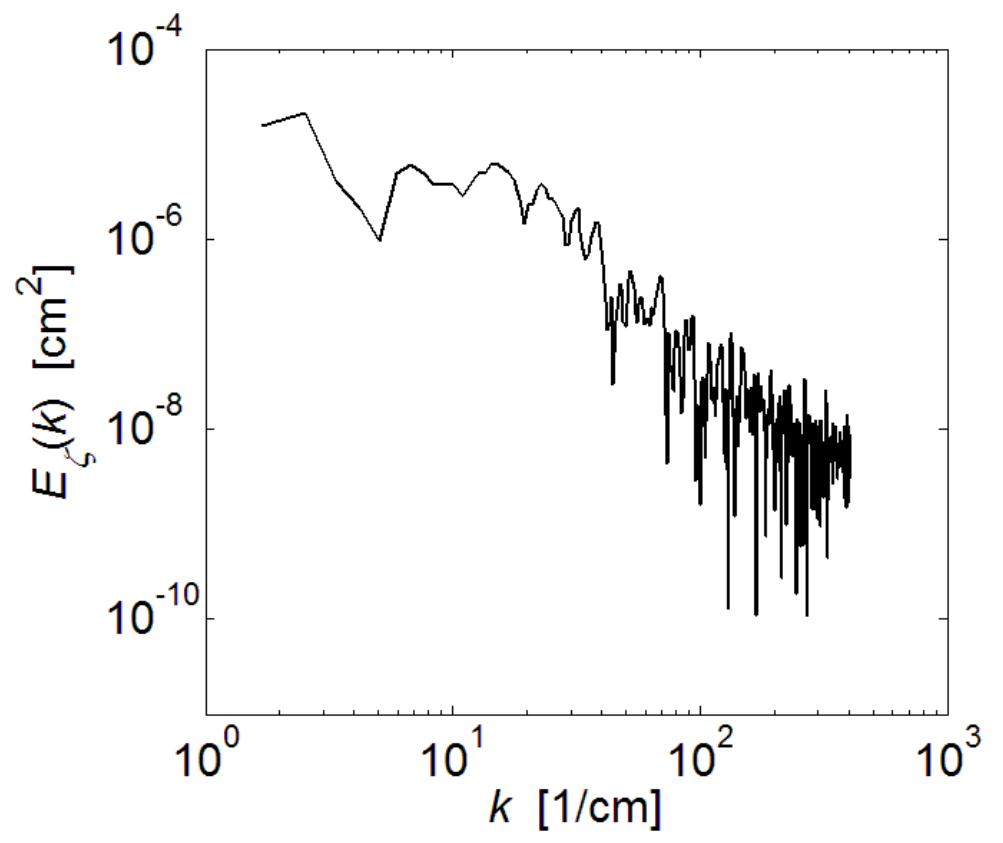

Figure 3.12 Spectrum of spanwise interfacial perturbations. 


\section{PARAMETERIZATION OF INITIAL CONDITIONS}

\subsection{Overview of Parameterization Methods}

A principle objective of this research is to quantify and to parameterize the initial conditions measurements presented in Section 3 so that they could be implemented in numerical simulations of the experiment. This has been accomplished through the spectral representation of the initial density (interfacial) and velocity fluctuations, rather than their pointwise values. The motivation for this is as follows. First, it allows for simulations using periodic boundary conditions in the homogeneous directions (directions not aligned with gravity) to be initialized with experimentallymeasured perturbations, while avoiding discontinuities at the boundaries. A spectral representation of the initial perturbations is also used so that a degree of stochastic abstraction could be achieved. This is desirable because all of the initial condition measurements were taken in a series of experiments. Thus, there is no assurance that the exact perturbation and phase distribution measured will occur in exactly the same fashion from one experiment to another, but statistical convergence of the initial perturbation spectra is possible. Each measurement from Section 3 is treated individually in the following sections.

It is important to recognize that the parameterization of the initial conditions in this investigation differs from most initializations listed in $\S 1.3$ and table 1.1 , in that isotropic initial perturbations are not assumed or implemented. For the water channel experiments, velocity or density field values are perturbed in orthogonal directions. In three-dimensional simulations using isotropic initial conditions, the initial perturbation spectrum is $E_{\zeta}\left(k_{2 D}\right)$, where $k_{2 D} \equiv \sqrt{k_{x}^{2}+k_{y}^{2}}$ and $k_{x}$ and $k_{y}$ are the wavenumbers in each homogeneous direction. Thus, the amplitudes of the interfacial

fluctuations $\widehat{\zeta}\left(k_{2 D}\right)$ are equal for any two wavevectors $k_{2 D}$ of the same length, by the 
definition of isotropy. The initial perturbation for an isotropic perturbation field is defined

$$
\zeta(\mathbf{x})=\int \widehat{\zeta}(\mathbf{k}) \mathrm{e}^{i \mathbf{k} \cdot \mathbf{x}} d \mathbf{k}
$$

To place equation (4.1) into context, it is rewritten as a product of two Fourier series in its discrete form

$$
\begin{aligned}
\zeta(x, y)= & \sum_{k_{x}, k_{y}}\left\{\operatorname{Re}\left[\widehat{\zeta}\left(k_{x}\right)\right] \cos \left(k_{x} x\right)-i \operatorname{Im}\left[\widehat{\zeta}\left(k_{x}\right)\right] \sin \left(k_{x} x\right)\right\} \\
& \times\left\{\operatorname{Re}\left[\widehat{\zeta}\left(k_{y}\right)\right] \cos \left(k_{y} y\right)-i \operatorname{Im}\left[\widehat{\zeta}\left(k_{y}\right)\right] \sin \left(k_{y} y\right)\right\}
\end{aligned}
$$

It is discernible from equation (4.2) that two cross terms are formed in the multiplication of two Fourier series resulting in the introduction of perturbations that are not aligned with either of the orthogonal basis vectors, $\hat{\mathbf{e}}_{x}$ and $\hat{\mathbf{e}}_{y}$. This is not the case in the water channel experiment or in the parameterization presented here, where all perturbations are aligned with either $\hat{\mathbf{e}}_{x}$ or $\hat{\mathbf{e}}_{y}$. Note that two-dimensional spectra are used to initialize Rayleigh-Taylor flows with two directions of statistical homogeneity (periodicity) instead of three-dimensional spectra, as used in the initialization of simulations of isotropic turbulence. In Rayleigh-Taylor flows, Fourier decomposition is not possible in inhomogeneous directions (directions aligned with gravity) because of non-periodic behavior of scalar values (i.e. density) in the $z$-direction.

\subsection{Streamwise Interfacial Perturbation}

The initial interfacial perturbation in the $x$-direction was obtained from the thermocouple measurements at $x=0.1 \mathrm{~cm}$ and at $x=1.0 \mathrm{~cm}$. The thermocouple measurements resulted in a trace of density values $\rho(x)$ at the centerline of the mixing layer. However, the spectral representation of these density values $\widehat{\rho}(k)$ alone are insufficient to produce a trace of the interfacial perturbation $\zeta(x)$. To accomplish 
this transformation, a relationship between $\rho(x)$ and $\zeta(x)$ must be assumed or hypothesized. The relationship used in this investigation was a parameterization of a perturbed, diffuse interface between two fluids of different densities, similar to that used by Cook and Dimotakis (2001),

$$
\rho(x, y, z)=\langle\rho\rangle+\frac{\Delta \rho}{2} \operatorname{erf}\left[\frac{z+\zeta(x, y)}{\varepsilon}\right],
$$

where $\Delta \rho=\rho_{1}-\rho_{2}, \varepsilon$ is the half-width of the diffusion layer between the two unmixed fluids, and erf $(x)$ is the error function. This parameterization of the density trace along the centerline is based upon the analytical solution to the diffusion equation (Mills 1999); however, one caveat applied to equation (4.3) is that diffusion is assumed to occur only in the $z$-direction. For a perturbed interface in the limit of $\widehat{\zeta}(k) \ll$ $2 \pi / k$, equation (4.3) is valid. Simplifying equation (4.3) for the one-dimensional measurements presented in $\S 3.1$, the relationship between the centerline $(z=0)$ density trace and the interfacial perturbation becomes

$$
\rho(x)=\langle\rho\rangle+\frac{\Delta \rho}{2} \operatorname{erf}\left[\frac{\zeta(x)}{\varepsilon}\right] .
$$

To relate $\zeta(x)$ to $\rho(x)$, a value of $\varepsilon$ must be chosen. It is possible to estimate an interfacial thickness from the heat diffusion equation, where $\varepsilon$ can be evaluated analytically as $\varepsilon=\sqrt{4 D t}$, where $D \approx 1.5 \times 10^{-3} \mathrm{~cm}^{2} / \mathrm{s}$ is the thermal diffusivity of water at $20^{\circ} \mathrm{C}$ (Mills 1999). This estimate is believed to be valid because of the minimal deformation of the two-fluid interface in the region of $0<x<1.0 \mathrm{~cm}$.

Given the discrete grid of a numerical simulation with periodic boundary conditions, the wavenumbers supported are limited to the number of integer wavelengths that exist in a given domain. The wavenumbers supported in each homogeneous direction by a periodic grid with $N$ grid points in each can be determined from equation (2.10) where, in this case, $L_{0}$ is the domain width of the numerical simulation and 
the supported modes $\operatorname{span} m=1,2, \ldots, N / 2-1$.

The wavenumbers supported by a given grid resolution are independent of the details of each experimental measurement, i.e. the sampling rate of an experiment and the grid spacing of a simulation might differ, resulting in differences between the wavenumbers measured and the wavenumbers supported numerically. To remedy this, the energy spectrum $E_{\zeta}(k)$ implemented in a numerical simulation was interpolated from the experimental data, using a linear interpolation of the two bounding measured energy values. Once the numerical energy spectrum was created from the interpolation scheme, the spectrum was scaled so that the total energy in the measured and numerical spectrum were equal. The energy is determined as

$$
E_{\zeta}=\int_{k_{\min }}^{k_{\max }} E_{\zeta}(k) d k
$$

The difference between the measured wavenumbers and numerically-implemented wavenumbers indicates that caution must be exercised when using equation (4.5). Table 4.1 shows the minimum and maximum wavenumbers for a typical thermocouple experiment and numerical simulation. Thus, the integration limits in equation (4.5) must contain only the overlapping wavenumber domain of the simulation and experiment.

For completeness, all numerical simulations were initialized with an initiallydiffuse interface of thickness $\delta=2 \varepsilon$. Joseph (1990) and Sandoval (1995) showed that for two miscible, incompressible fluids, the divergence of the velocity field

$$
\begin{aligned}
\boldsymbol{\nabla} \cdot \mathbf{u} & \neq 0 \\
& =-\boldsymbol{\nabla} \cdot\left(\frac{D}{\rho} \nabla \rho\right)
\end{aligned}
$$

where $D$ is the mass diffusivity. Thus, because an initially diffuse interface exists in 


\begin{tabular}{ccc}
\hline & $k_{\min } \mathrm{cm}^{-1}$ & $k_{\max } \mathrm{cm}^{-1}$ \\
\hline \hline Typical Experiment & 0.025 (Density) & 374 (Density) \\
$U_{m}=4.2 \mathrm{~cm} / \mathrm{s}$ & 0.037 (Velocity) & 22.4 (Velocity) \\
Two-dimensional DNS & & \\
$1024 \times 1024$ grid points & 0.196 & 101 \\
$32 \mathrm{~cm} \times 32 \mathrm{~cm}$ & & \\
Three-dimensional DNS & & 50.3 \\
$256 \times 128 \times 256$ grid points & 0.393 & \\
$16 \mathrm{~cm} \times 10 \mathrm{~cm} \times 16 \mathrm{~cm}$ & & \\
\hline
\end{tabular}

Table 4.1 Minimum and maximum wavenumbers for a typical experiment and for representative two- and three-dimensional DNS.

the parameterization of the initial density field, there must also exist a velocity field

$$
\mathbf{u}=-\frac{D}{\rho} \nabla \rho .
$$

All of the simulations presented in the current investigation, regardless of their initialization, satisfy the criteria listed above by imposing the velocity field specified by equation (4.7).

\subsection{Streamwise Velocity Perturbation}

Implementation of an initial velocity perturbation requires a different approach than that used for the interfacial perturbations. As shown by equations (4.3) and (4.7) in $\S 4.4$, the perturbation at the centerplane $\zeta(x, y)$ is the only quantity required to initialize the density field $\rho(x, y, z)$ and the velocity field $\mathbf{u}(x, y, z)$. For the case of initial velocity conditions, specification of the entire two-dimensional velocity field is accomplished by the sum of three independent velocity fields, which are discussed 
below.

To form the two-dimensional velocity field, the first information used from the experimental measurements was the energy spectrum of the vertical velocity component at $x=0.5 \mathrm{~cm}$ from the splitter plate. Assuming a uniform distribution of random phases $\varphi(k)$, the Fourier amplitudes of each wave $\widehat{w}(k)=\sqrt{E_{w}(k)}$ were calculated. Like the numerically implemented, streamwise, interfacial perturbation spectrum, values of $\widehat{w}(k)$ were interpolated from the experimentally-measured values. Once the amplitude and phase of each wave at the centerline was calculated, a two-dimensional, perturbed velocity potential field is created (Drazin \& Reid 2004):

$$
\phi(x, y, z)=\sum_{k=k_{\min }}^{k_{\max }} \frac{\widehat{w}(k)}{k} \sin [k x+\varphi(k)] \mathrm{e}^{-k|z|},
$$

where $\varphi(k)$ is the phase associated with each mode $\widehat{w}(k)$. The velocity field associated with the perturbed potential field is created by

$$
\mathbf{u}=\nabla \phi
$$

For completeness, it is noted that the initial velocity field was created by differentiating the potential field using a fourth-order central-differencing scheme in both coordinate directions. Boundary conditions in the $x$-direction were assumed to be periodic, eliminating all discontinuities in $\phi$. However, discontinuities in $\phi$ exist in the $z$-direction at the top and bottom boundaries and at the centerline. To account for the discontinuities, a one-sided, fourth-order differencing scheme was used. Images of sample initial $u$ velocity and $w$ velocity fields generated from a potential $\phi$ are shown in figure 4.1 .

To complete the parameterization of the initial velocity conditions, the initial velocity deficit that occurs at the trailing edge of the splitter plate was also included 

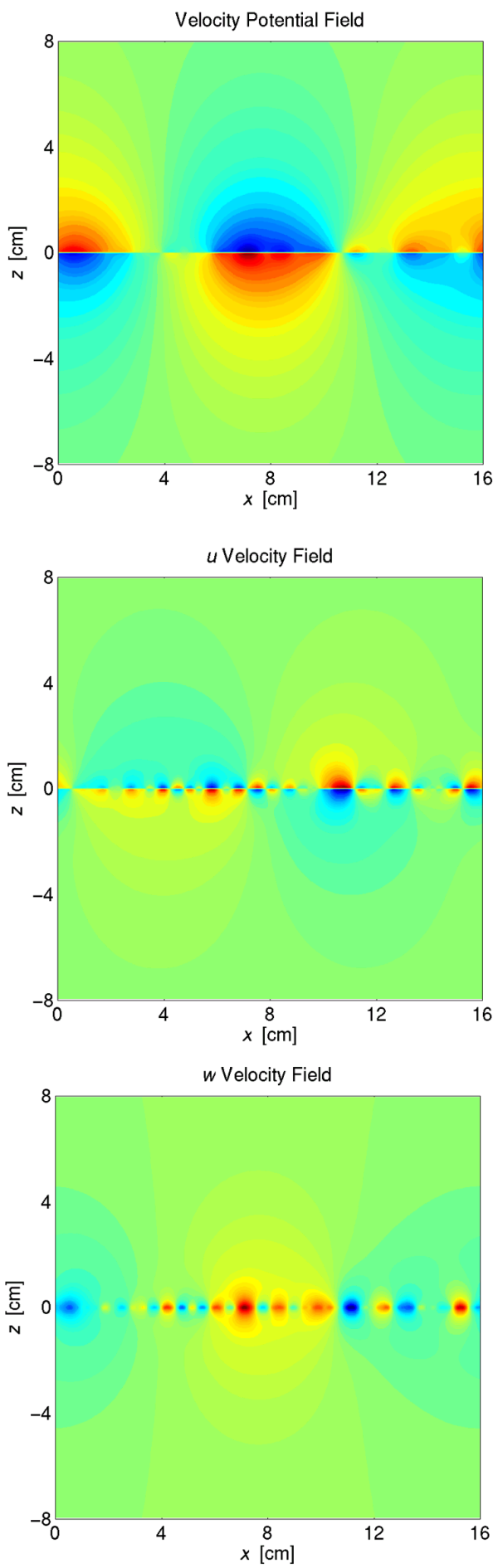

Figure 4.1 Illustration of the initial velocity potential field (top), $u$ velocity field (middle), and $w$ velocity field (bottom). All images are from a $256 \times 256$ DNS with domain size of $16 \mathrm{~cm} \times 16 \mathrm{~cm}$. 
in the initial two-dimensional velocity field. As the numerical simulations do not include any mean advective velocity, the velocity deficit was parameterized by the inclusion of a small, negative $u$ velocity at the centerplane, which decays to zero away from the centerplane. A simple mathematical model is proposed to model the velocity deficit, such that several key criteria are satisfied. First, the parameterization must satisfy mass continuity, such that $\boldsymbol{\nabla} \cdot \mathbf{u}^{*}=0$, where $\mathbf{u}^{*}=u^{*} \hat{\mathbf{e}}_{x}+v^{*} \hat{\mathbf{e}}_{y}+w^{*} \hat{\mathbf{e}}_{z}$ is the velocity deficit field. Second, the model must satisfy the boundary conditions $u(z)=0$ as $z \rightarrow \pm \infty$. Two possibilities for the parameterization of the initial velocity deficit are

$$
\left(u^{*}, v^{*}, w^{*}\right)=\left(u_{\text {def }} \mathrm{e}^{-|z| / L}, 0,0\right)
$$

and

$$
\left(u^{*}, v^{*}, w^{*}\right)=\left(u_{d e f} \mathrm{e}^{-z^{2} / L^{2}}, 0,0\right)
$$

where $u_{\text {def }}$ is a constant negative velocity, and $L \approx 0.3 \mathrm{~cm}$ is a lengthscale selected so that the implemented velocity profile matches the experimentally-measured profile. Both parameterizations satisfy continuity, in that $\boldsymbol{\nabla} \cdot \mathbf{u}^{*}=0$, and both go to zero as $|z| \rightarrow \infty$. For this investigation, equation (4.11) was used to parameterize the initial velocity deficit because it is a smooth function over the domain $z=(-\infty, \infty)$.

The final portion of the velocity field was created from equation (4.7). All three portions of the initial velocity field are combined to form the final initial, twodimensional velocity field such that

$$
\mathbf{u}(\mathbf{x}, t=0)=\nabla \phi+\mathbf{u}^{*}-\frac{D}{\rho} \nabla \rho
$$

By construction, equation (4.7) is satisfied and, therefore, mass conservation was also satisfied. 


\subsection{Spanwise Interfacial Perturbation}

The spanwise interfacial perturbation is possibly the simplest to implement, in that this perturbation is measured directly from a single PLIF image and no transformations are required. The interfacial perturbation in the $y$-direction is formed from the inverse Fourier transform of $\widehat{\zeta}(k)=\sqrt{E_{\zeta}(k)}$ assuming a uniform distribution of random phases. Like the streamwise interfacial and velocity perturbations, the

numerically-implemented values of $\widehat{\zeta}(k)$ are interpolated from the measured values of $\widehat{\zeta}(k)$.

The only assumption required to implement the spanwise interfacial perturbation is the need to extend the lower bound of wavenumbers measured to match the domain of a numerical simulation. The minimum wavenumber measured from the PLIF image is $k \approx 0.82 \mathrm{~cm}^{-1}$, corresponding to a wavelength $\lambda \approx 7.7 \mathrm{~cm}$. For numerical domain sizes greater than $7.7 \mathrm{~cm}$, an unphysical periodicity can be added to the flow. While simulations in which isotropic initial perturbations at the two-fluid interface are assumed may incorporate wavelengths less than the domain width of interest, the anisotropic initial conditions in the investigation prohibit this. To overcome this limitation (albeit physical in nature) the modes with wavenumbers $k<0.82 \mathrm{~cm}^{-1}$ are given an assumed energy and phase. This assumption is validated by the existence of a slight bowing of the splitter plate at its trailing edge, which can be seen on the right-hand side of figure 3.11. While this bowing effect is not a periodic perturbation that can be implemented in the numerical simulation, it does introduce a perturbation below the lowest measured wavenumber. While this assumption is stated explicitly, the effects of this assumption on the solution within the time of interest have yet to be determined and understood. 


\section{DIRECT NUMERICAL SIMULATIONS AND COMPARISONS TO EXPERIMENTAL DATA}

\subsection{Preliminaries}

The use of direct numerical simulations (DNS) for this study was chosen for several reasons. First, the water channel experiment is an ideal candidate for DNS, in that the computational expense associated with DNS scales as $R e^{3}$. This renders very high Reynolds number experiments currently impossible to simulate using DNS, such as the experiments performed by Read (1984) and by Dimonte and Schneider (1996) inaccessible. Depending on the Reynolds number definition, Ramaprabhu and Andrews (2004a) report values of $R e \approx 1000-1450$ at $\tau=1.21$. The simulation of a flow with such a Reynolds number is achievable using DNS without the need for a large allocation of computing resources. Furthermore, the physical size of the experiment $(20 \mathrm{~cm} \times 32 \mathrm{~cm}$ cross-section) is amenable to DNS, allowing for the full range of scales to be represented on a discrete grid. Also, statistically-stationary turbulence and mixing statistics have been measured in the water channel experiment, allowing for direct statistical comparisons to be made between experimental and numerical simulation data. In particular, accurate measurements of the degree of molecular mixing require the ability to resolve the smallest scales of motion where mass diffusion is most effective. The ability to measure the statistics of fluctuating quantities in the water channel also allows the measurement, parameterization, and use of realistic initial perturbations in DNS.

Another theme of the current numerical study is the determination of the quantitative differences between two- and three-dimensional simulations of Rayleigh-Taylor instability growth and mixing. This goal is motivated by the desire to provide more predictive models without the need for computationally-expensive, three-dimensional 
calculations of flows that occur in complex systems, such as in high-energy density applications (Kilkenny et al. 1994; Haan et al. 1995; Lindl 1998). Results will be shown comparing two- and three-dimensional simulations initialized with analogous, experimentally-measured initial conditions. Differences between the results of the simulations will be highlighted and the underlying physical mechanisms that are responsible for these differences will be discussed.

\subsection{Numerical Scheme}

The numerical simulations for this research have been performed using a spectral/compact finite-difference code developed at the Lawrence Livermore National Laboratory (LLNL) primarily for the direct and large-eddy simulation of RayleighTaylor instability-generated turbulence (Cook \& Dimotakis 2001; Cook et al. 2004). The code solves the mass, momentum, and species diffusion equations on a Cartesian grid. For the present investigation, the code was used to perform DNS, in which the entire range of scales are resolved within a turbulent flow. Spatial derivatives are computed using spectral methods in the homogeneous $x$ - and $y$-directions and tenth-order compact finite-differencing in the inhomogeneous $z$-direction. Time is advanced using a third-order Adams-Bashforth-Moulton scheme for all timesteps with the exception of the first, which employs a forward Euler scheme. To avoid complex inflow and outflow boundary conditions, the numerical simulations had no mean flow component. Boundary conditions in the homogeneous directions were periodic and no-slip/nopenetration in the vertical direction. The code is parallelized using the MPI library and was tuned for use on large-scale computing facilities across many processors. The simulations were performed on the Blue Pacific supercomputer at LLNL. 
The numerical scheme solves the mass conservation equation,

$$
\frac{\partial \rho}{\partial t}+\frac{\partial}{\partial x_{j}}\left(\rho u_{j}\right)=0
$$

and the momentum conservation equation,

$$
\frac{\partial}{\partial t}\left(\rho u_{i}\right)+\frac{\partial}{\partial x_{j}}\left(\rho u_{i} u_{j}\right)=\rho g_{i}-\frac{\partial p}{\partial x_{i}}+\frac{\partial \sigma_{i j}}{\partial x_{j}}
$$

where

$$
\sigma_{i j}=\mu\left(\frac{\partial u_{i}}{\partial x_{j}}+\frac{\partial u_{j}}{\partial x_{i}}-\frac{2}{3} \delta_{i j} \frac{\partial u_{k}}{\partial x_{k}}\right),
$$

is the viscous stress tensor and

$$
\mu \equiv \frac{\left(\nu_{1}+\nu_{2}\right)}{2} \frac{\left(\rho_{1}+\rho_{2}\right)}{2}
$$

is the dynamic viscosity. In equations (5.1)-(5.3),

$$
\left(u_{1}, u_{2}, u_{3}\right)=(u, v, w)
$$

For all of the numerical simulations, gravity acts in the vertical direction such that $g_{1}=g_{2}=0$ and $g_{3}=-981 \mathrm{~cm} / \mathrm{s}^{2}$. The governing equations describe an incompressible, variable-density flow. The two fluid streams have been treated as miscible and the mass diffusion of one fluid into the other was calculated by the mass fraction conservation equation assuming constant diffusivity $D$ :

$$
\frac{\partial}{\partial t}\left(\rho Y_{\alpha}\right)+\frac{\partial}{\partial x_{j}}\left(\rho Y_{\alpha} u_{j}\right)=\frac{\partial}{\partial x_{j}}\left(\rho D \frac{\partial Y_{\alpha}}{\partial x_{j}}\right) .
$$

The numerical scheme is quasi-incompressible in the sense that pressure waves are decoupled from the governing equations and are neglected. The numerical algorithm used in this investigation has been shown to match the growth rate of a single-mode instability as predicted by linear theory for small-amplitude perturbations (Cook \& 
Dimotakis 2001; Cook et al. 2004). To mitigate aliasing errors and oscillations due to numerical instabilities introduced by the numerical algorithm, filtering was performed. Additional details regarding the filtering techniques can be found in Cook and Dimotakis (2001) and Cook et al. (2004).

\subsection{Summary of Direct Numerical Simulations}

DNS were performed to determine the optimum method of parameterization of the measured initial conditions. Simulation parameters were chosen such that they matched the experimental values of density, viscosity, mass (thermal) diffusivity, and physical domain size. A summary of these parameters is given in table 5.1. The table shows the same values listed in table 2.1 with one subtle difference. The numerical algorithm employed solves the diffusion of mass species, whereas heat diffusion occurs in the experiment. It is assumed that $\operatorname{Pr}=S c=7$ in all simulations to calculate the molecular diffusion of one fluid into another.

A summary of the parameterized initial conditions is given in table 5.2. Both three-dimensional simulations employ the same interfacial perturbation in the spanwise direction, while differing only in the streamwise perturbations. Also, due to numerical stability and resolution issues, the domain sizes of the three-dimensional simulations are nominally half of the domain size for their respective two-dimensional simulations.

\subsection{Qualitative Results}

The evolution of the density field and growth of a Rayleigh-Taylor mixing layer in a two-dimensional DNS with initial velocity perturbations is shown in figure 5.1. The two unmixed fluids (red and blue) are initially separated by a perfectly flat interface. 


\begin{tabular}{cc}
\hline Parameter & Value \\
\hline$\rho_{1}$ & $0.9986 \mathrm{~g} / \mathrm{cm}^{3}$ \\
$\rho_{2}$ & $0.9970 \mathrm{~g} / \mathrm{cm}^{3}$ \\
$A$ & $7.5 \times 10^{-4}$ \\
$g$ & $-981 \mathrm{~cm} / \mathrm{s}^{2}$ \\
$\mu_{1}$ & $0.009 \mathrm{~g} /(\mathrm{cm} \mathrm{s})$ \\
$\mu_{2}$ & $0.011 \mathrm{~g} /(\mathrm{cm} \mathrm{s})$ \\
$S c$ & 7.0 \\
$L_{x} \times L_{z}(2 \mathrm{D})$ & $32 \mathrm{~cm} \times 32 \mathrm{~cm}$ \\
$N_{x} \times N_{z}(2 \mathrm{D})$ & $1024 \times 1024$ \\
$L_{x} \times L_{y} \times L_{z}(3 \mathrm{D})$ & $16 \mathrm{~cm} \times 10 \mathrm{~cm} \times 16 \mathrm{~cm}$ \\
$N_{x} \times N_{y} \times N_{z}(3 \mathrm{D})$ & $256 \times 128 \times 256$ \\
\hline
\end{tabular}

Table 5.1 Two- and three-dimensional DNS parameters.

\begin{tabular}{ccc}
\hline Simulation & $\begin{array}{c}x \text {-direction } \\
\text { perturbation }\end{array}$ & $\begin{array}{c}y \text {-direction } \\
\text { perturbation }\end{array}$ \\
\hline \hline Initial Density Conditions & $\begin{array}{c}\text { Interfacial } \\
\text { (Thermocouple) }\end{array}$ & \\
$3 \mathrm{D}$ & Interfacial & $\begin{array}{c}\text { Interfacial } \\
\text { (PLIF) }\end{array}$ \\
Initial Density Conditions & (Thermocouple) & \\
$2 \mathrm{D}$ & Velocity & \\
Initial Velocity Conditions & $($ PIV $)$ & Interfacial \\
$3 \mathrm{D}$ & Velocity & $($ PLIF $)$ \\
\hline
\end{tabular}

Table 5.2 Summary of parameterized initial conditions for two-dimensional and three dimensional DNS. 
This flat interface is only used for initial velocity perturbation simulations and not for initial interfacial perturbation simulations. The images show the evolution of smallscale perturbations at $\tau=0.152$, saturation of the initial perturbation growth rate, and the onset of secondary Kelvin-Helmholtz instabilities by $\tau=0.758$, and the development of a strongly-nonlinear flow at later times, $\tau=1.36$ and $\tau=1.97$. The use of the term turbulence is avoided because of the two-dimensionality of this flow, in which three-dimensional effects, such as vortex stretching, are not present. Aside from the absence of vortex stretching, Schilling et al. (2004a) have shown fundamental differences between two- and three-dimensional MILES (using the weighted essentially nonoscillatory method) of Richtmyer-Meshkov generated turbulence, where RichtmyerMeshkov instabilities represent the special case of impulse-driven Rayleigh-Taylor flows. Schilling et al. reported significant differences between rates of dilatation and transport of vorticity and the transport and production of turbulent kinetic energy. Both the magnitude and distribution within the mixing layer of these processes were shown to vary significantly between two- and three-dimensional simulations.

The evolution of a mixing layer from a three-dimensional DNS is shown in figures 5.2 and 5.3 , where the evolution of the $f_{1}=0.5$ isosurface is plotted. The initial distortion of the two-fluid interface, as well as the anisotropic nature of the perturbation, can be seen at early times $(\tau=0.304)$. As in the two-dimensional simulation, as the initial perturbation saturates and Kelvin-Helmholtz instabilities begin to form by $\tau=0.607$. The onset of the three-dimensionality of the flow also begins as individual bubbles emerge from the nearly two-dimensional ripples. At the later stages of the flow, the two-fluid interface shown by the isosurfaces is no longer a single smooth surface: a more complicated, strongly-nonlinear flow has emerged. Both of the twoand three-dimensional DNS shown in figures 5.1-5.3 were initialized with the same velocity spectrum in the $x$-direction. However, the three-dimensional DNS was also 

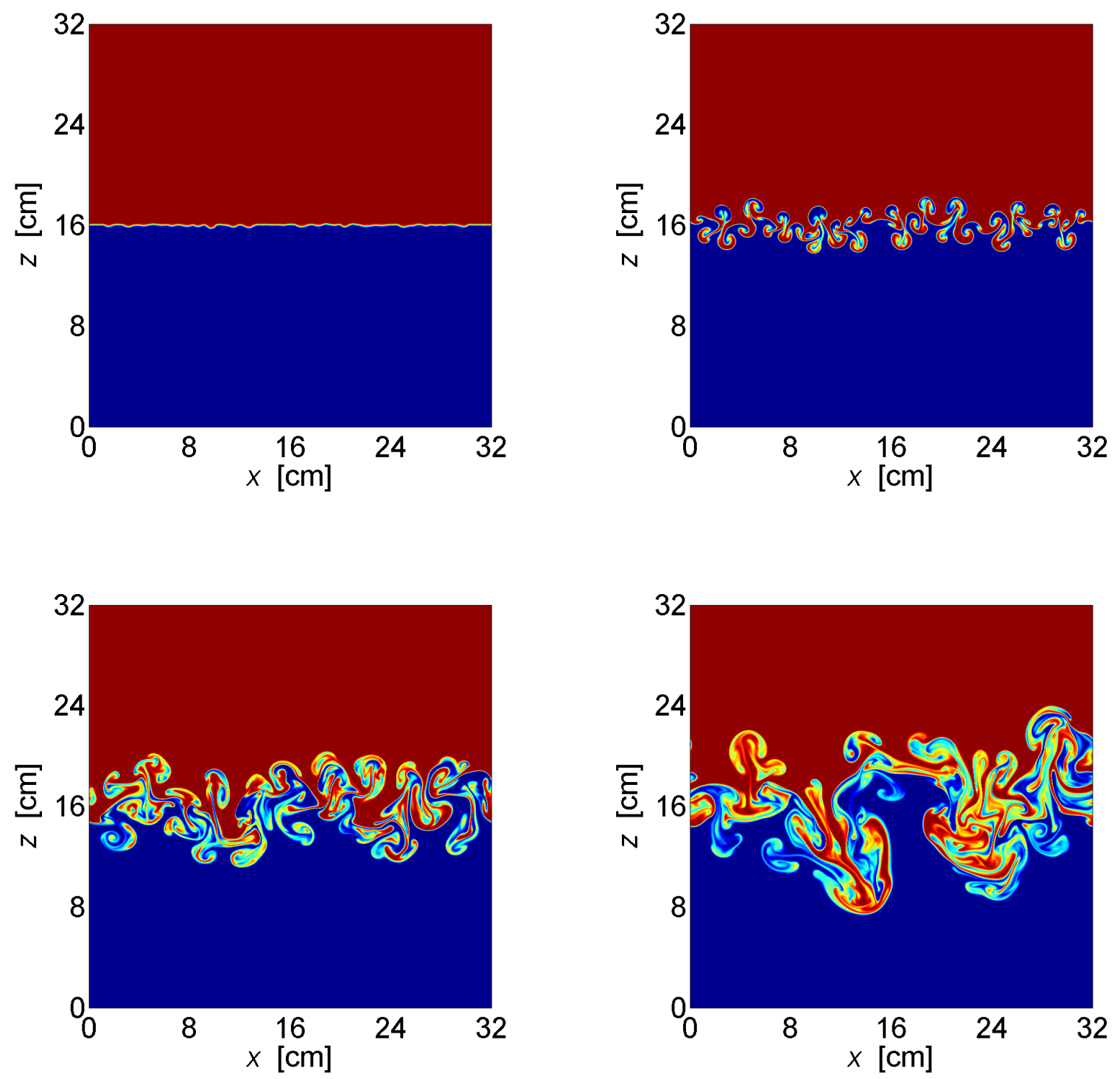

Figure 5.1 Evolution of the density field from a two-dimensional DNS with initial velocity perturbations. The red and blue represent the two unmixed fluids and intermediate shades represent mixed fluids. Images are shown at $\tau=0.152$ (top left), $\tau=0.758$ (top right), $\tau=1.36$ (bottom left), and $\tau=1.97$ (bottom right). 

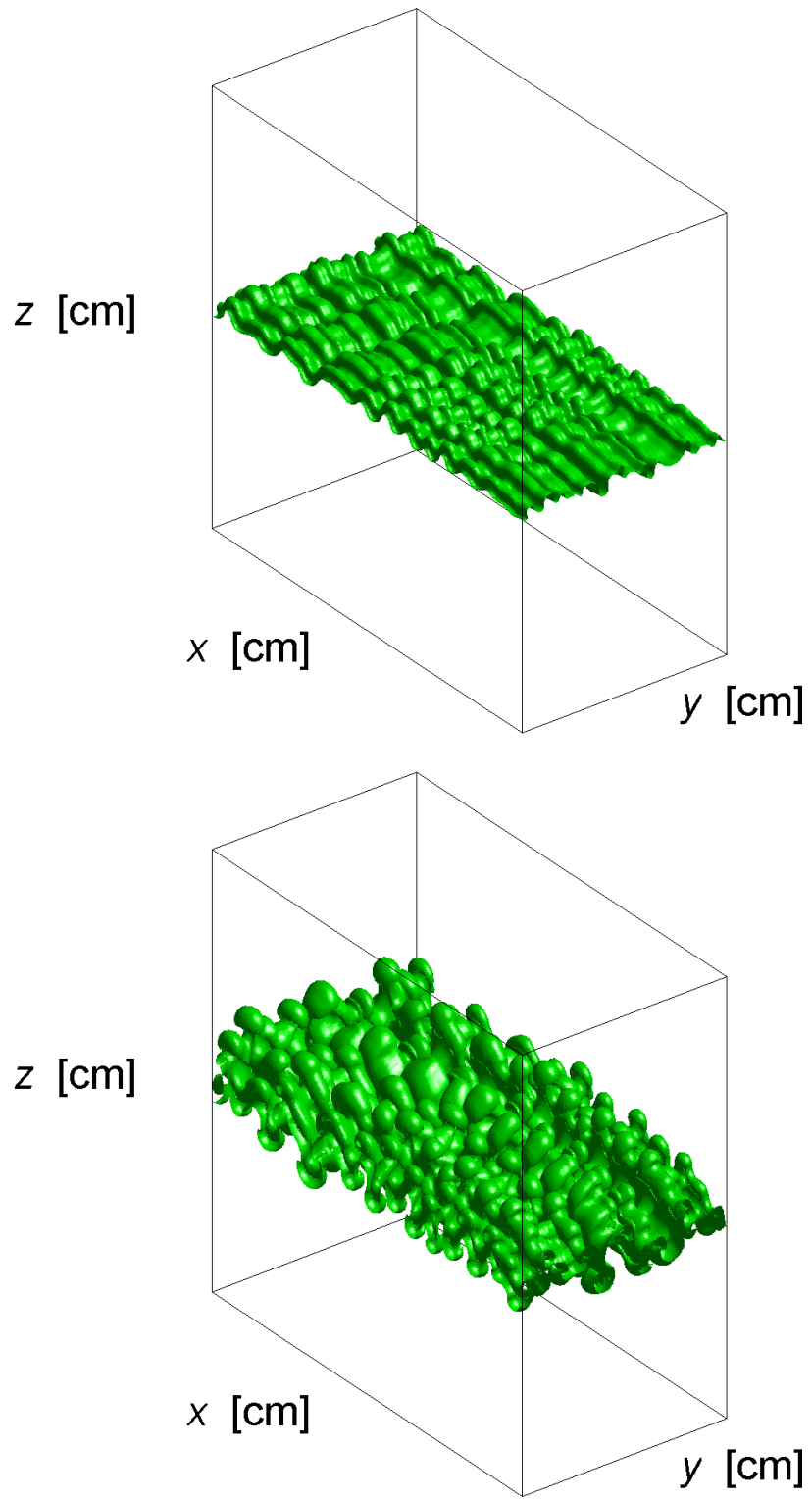

Figure 5.2 Evolution of the $f_{1}=0.5$ isosurface from a three-dimensional DNS with initial velocity and interfacial perturbations. Images are shown at $\tau=0.304$ (top) and $\tau=0.607$ (bottom). 

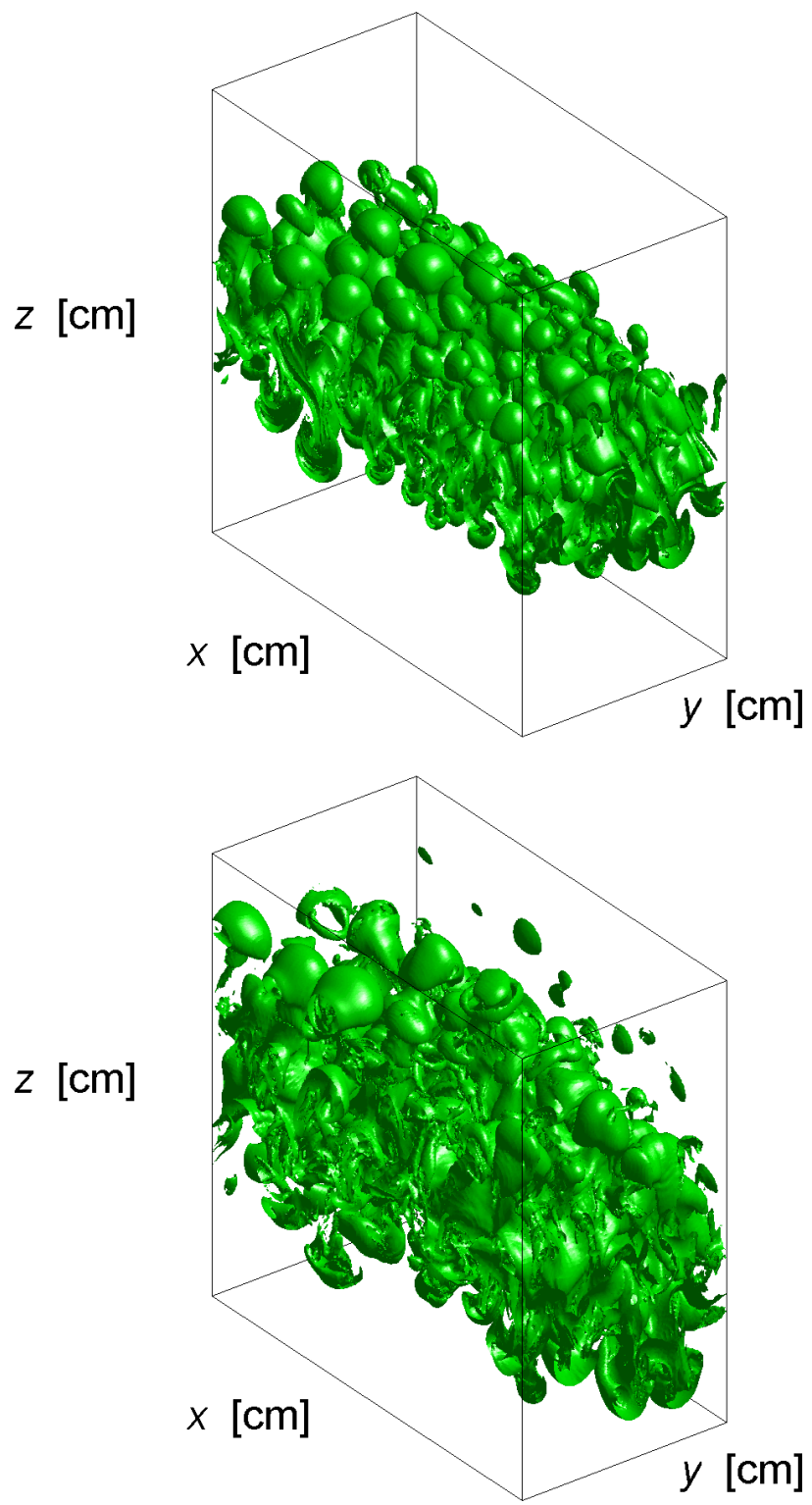

Figure 5.3 Evolution of the $f_{1}=0.5$ isosurface from a three-dimensional DNS with initial velocity and interfacial perturbations. Images are shown at $\tau=0.910$ (top) and $\tau=1.21$ (bottom). 
initialized with a measured interfacial perturbation in the $y$-direction, which is not represented in the two-dimensional DNS.

A qualitative comparison between the evolution of the water channel experiment and DNS is possible by considering a slice of the density field in the $x z$-plane. Figure 5.4 shows a PLIF image of the experiment centered at approximately $x=35 \mathrm{~cm}$ downstream at $\tau \approx 1.2 .{ }^{1}$ Cross-sectional slices in the $x z$-plane of the density field from a two- and three-dimensional DNS are also shown with approximately the same time of development. Both experiments and simulations were initialized with stochastic initial conditions, so that an exact comparison is not feasible. However, it is possible to qualitatively compare both large- and small-scale features of the flow using these images. First, all three images exhibit large-scale structures (bubble widths) of approximately the same width. The greatest difference between the images is the range of scales present in the density field. Both the experimental PLIF image and threedimensional DNS image exhibit a broad range of detail in the small scales, whereas the two-dimensional DNS does not. Presumably, this is due to the absence of vortex stretching and turbulent transport in the third spatial dimension as a mechanism for the creation of smaller scales.

While both the experimental PLIF image and the three-dimensional DNS exhibit similar fine scales within the density field, it appears that the PLIF image contains sharper density gradients than the simulation. In fact, the true density field is somewhere in between the two images. In the water channel, the active scalar driving the buoyancy-driven mixing is the temperature difference between the two fluids, where $\operatorname{Pr} \approx 7$ for the water temperatures used in the experiment. However, the fluorescing dye-tracer injected into the top water stream does not diffuse at the same rate as tem-

\footnotetext{
${ }^{1}$ PLIF image courtesy of W. N. Kraft.
} 


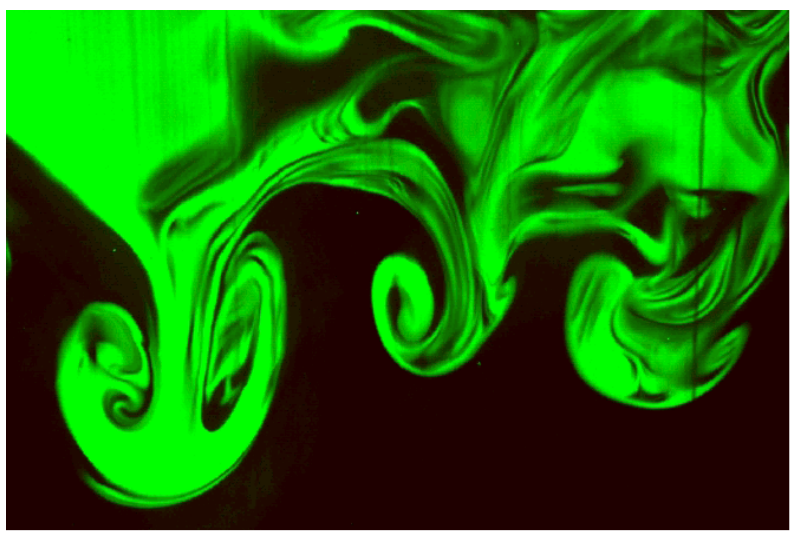

(a)

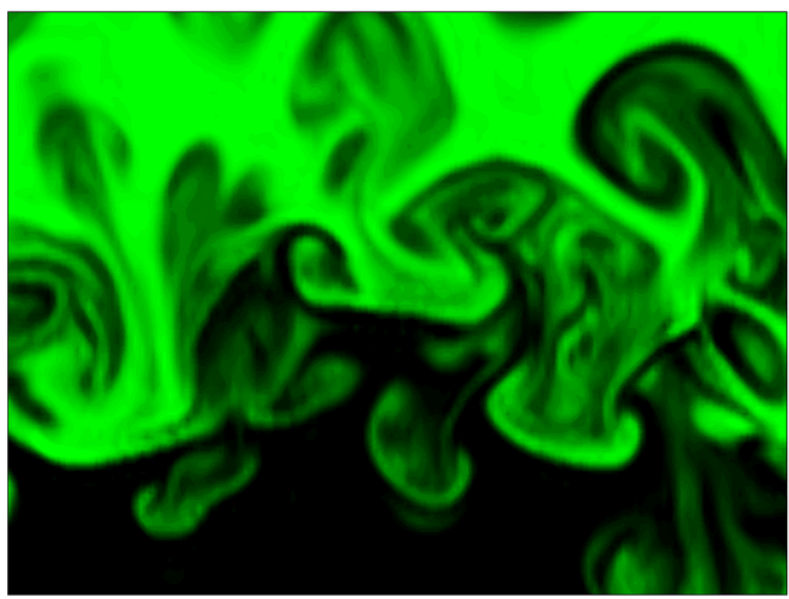

(b)

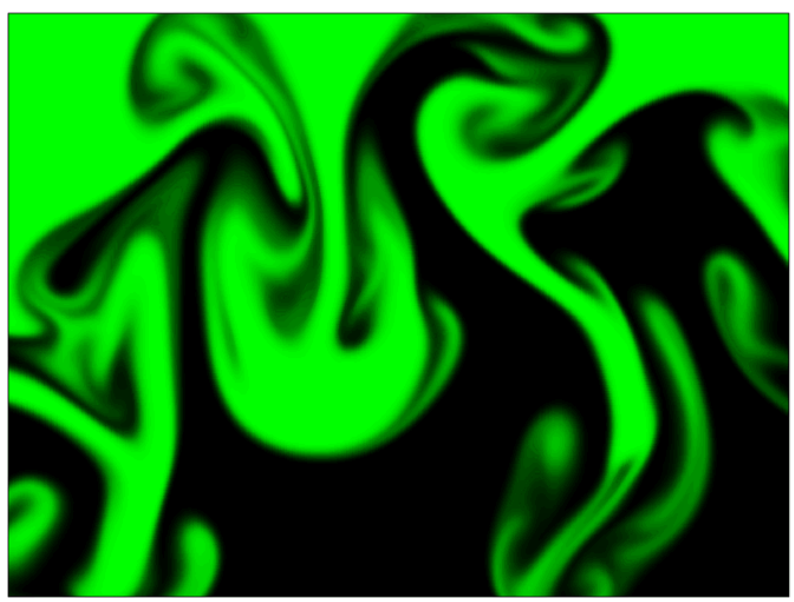

(c)

Figure 5.4 Comparison of slice of (a) PLIF image from experiment and PLIF-rendered slices of density field from (b) 3D initial velocity/density conditions DNS and (c) $2 \mathrm{D}$ initial velocity conditions DNS at $\tau \approx 1.2$. All images are approximately $7 \mathrm{~cm} \times 5.25 \mathrm{~cm}$. Experimental image courtesy of $\mathrm{W}$. N. Kraft. 
perature, as $S c \sim 1000$. This results in a Lewis number $L e \equiv S c / P r \sim 100$. Thus, as $\tau$ increases, the difference between the density (temperature) field and the scalar field of dye concentration diverges. On the other hand, the image from the DNS does not capture the fine-scale structure. This is due to the fact that the current three-dimensional DNS appears to be slightly under-resolved because of the need to apply filtering to mitigate aliasing errors as $R e \rightarrow 1000$. This resulted in a smoothing of the density gradients at the two-fluid interface. Integral-scale Reynolds numbers for the simulations will be discussed further in $§ 5.5$.

One unique aspect of this research is that these are the first simulations of $a$ Rayleigh-Taylor flow to implement fully-anisotropic, fully-measured, three-dimensional initial conditions. Figure 5.5 shows the evolution of the centerplane density and vertical velocity field for various development times. The anisotropy of the initial conditions is readily distinguished at $\tau=0$, where the interfacial (density) and vertical velocity $\left(w^{\prime}\right)$ perturbations are initially orthogonal. However, as $\tau$ increases, nonlinear interactions between the orthogonal perturbations begin to create energy content in wavevectors not aligned with $\hat{\mathbf{e}}_{x}$ and $\hat{\mathbf{e}}_{y}$. These images suggest that the initial conditions are a strong influence on the early stages of development, but at late-time, three-dimensional turbulent transport of momentum and density drive the mixing layer towards a state of isotropy in the homogeneous directions. At the latest time shown, $\tau=1.21$, a difference in the detail of the density and velocity fields is noticeable. It is speculated that this difference is the effect of $S c>1$, where the diffusion of velocity gradients by viscosity dominates the diffusion of density gradients by mass diffusion. This will discussed further in $§ 5.6$. 

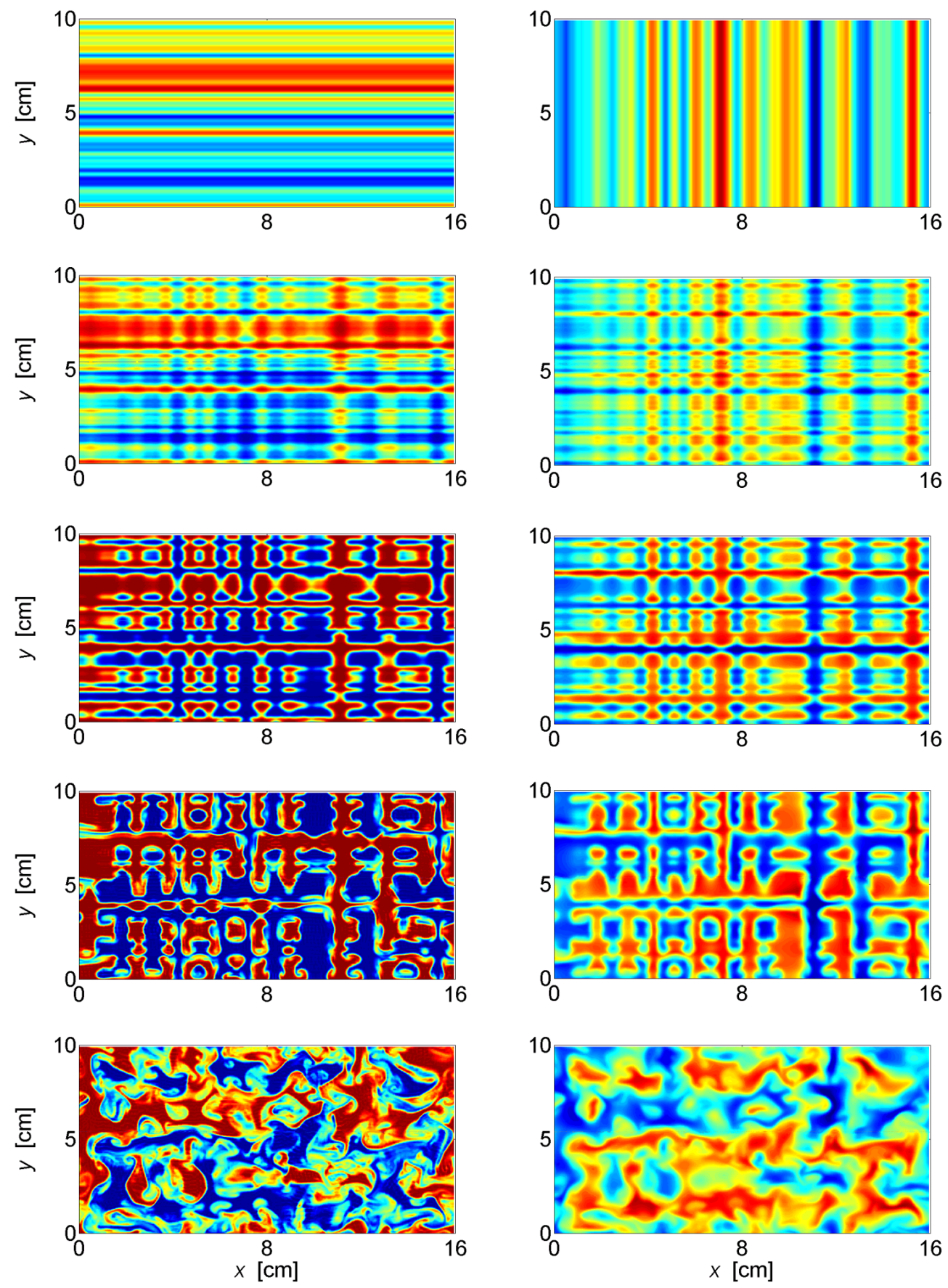

Figure 5.5 Time-evolution of the centerplane density (left column) and vertical velocity fields (right column). Images from top to bottom are at $\tau=0,0.076,0.304,0.607,1.21$. 


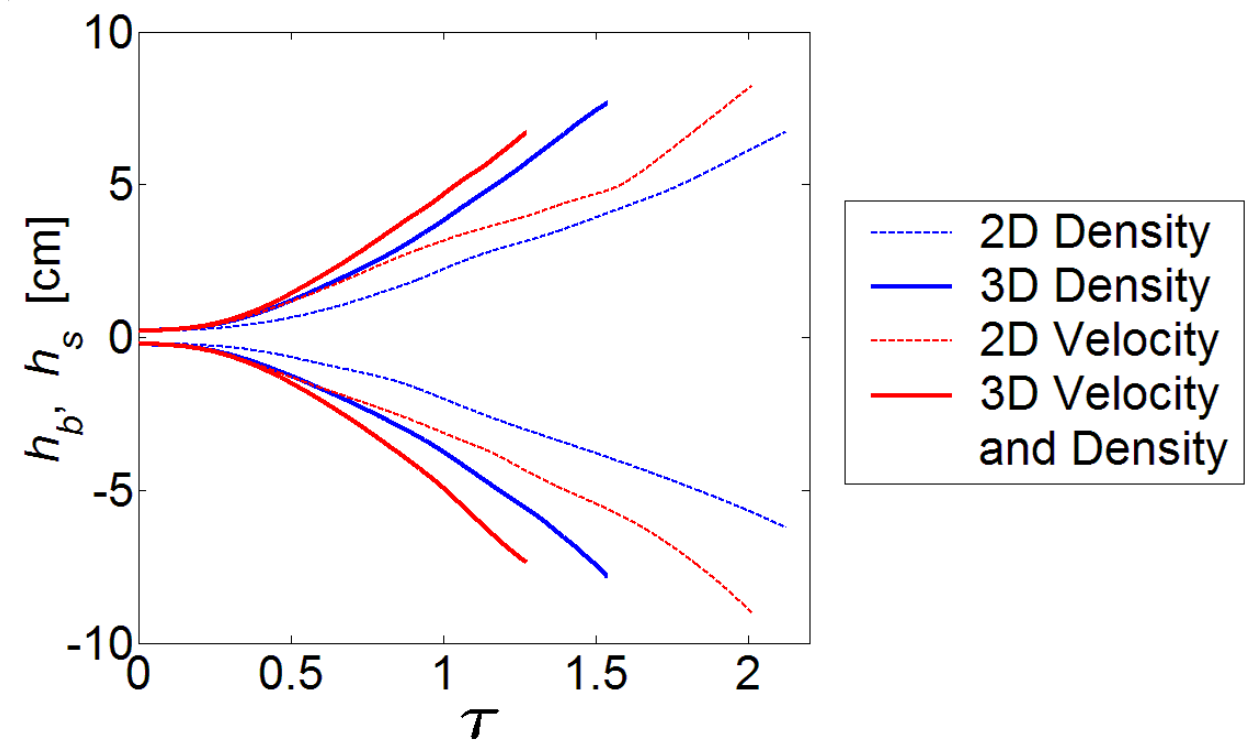

Figure 5.6 Time-evolution of the bubble and spike fronts, $h_{b}$ and $h_{s}$, from two- and three-dimensional DNS.

\subsection{Integral-Scale Results}

A canonical measure of the growth of a Rayleigh-Taylor driven mixing layer is the extent of interpenetration of the two fluids, typically quantified by measuring the distance between the centerplane $(z=0)$ and the plane in which $\left\langle f_{1}\right\rangle=0.05,0.95$. This is typically referred to as the "bubble" $h_{b}$ and "spike" $h_{s}$ amplitudes or heights, where the bubbles are the lighter rising fluid and the spikes are the heavier falling fluid. This nomenclature of bubbles and spikes originates from the $A \rightarrow 1$ case in which a significant asymmetry exists between the growth of the rising and falling structures (Youngs 1984). Figure 5.6 shows the evolution of $h_{b}$ and $h_{s}$ for DNS initialized with interfacial and/or velocity perturbations. The evolution of the total width of the mixing layer $h \equiv h_{b}+h_{s}$ is shown in figure 5.7.

Figures 5.6 and 5.7 show several trends. First, for the domain size and time 


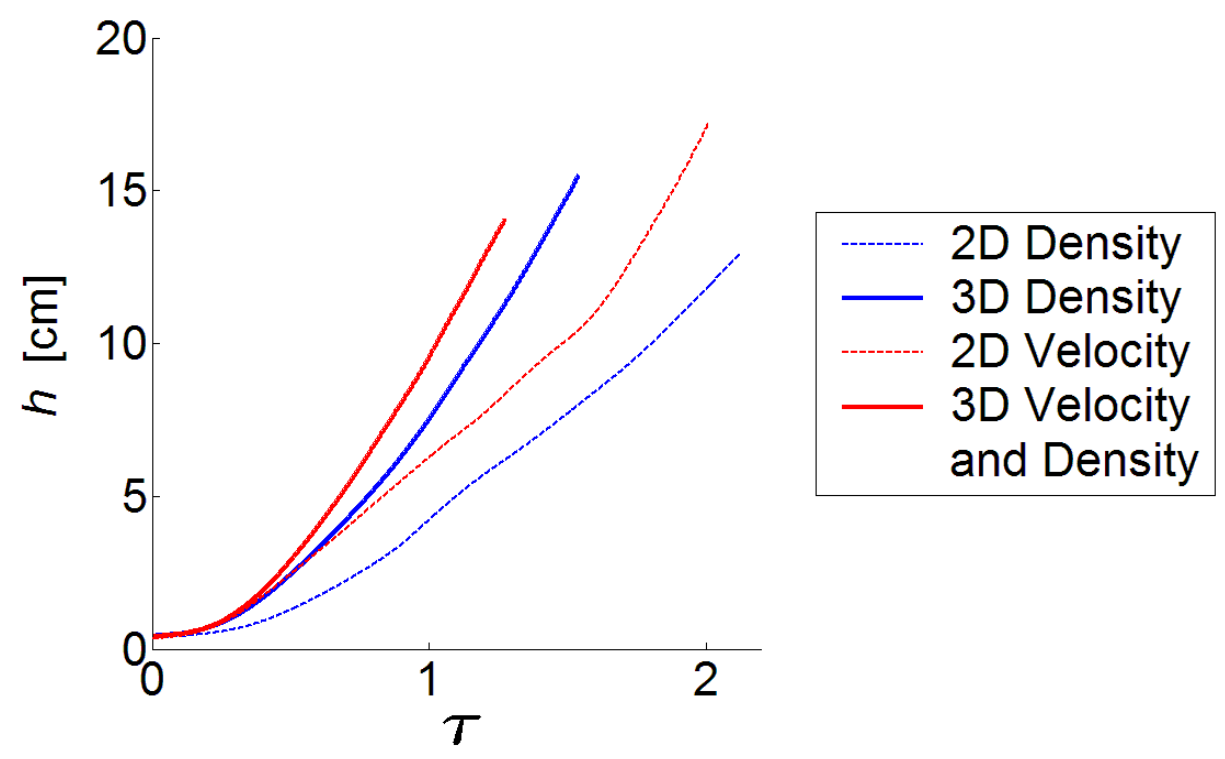

Figure 5.7 Time-evolution of the mixing layer width $h$ with respect to non-dimensional time.

of interest, both three-dimensional DNS exhibited larger mixing layer growth rates than the two-dimensional DNS. The difference in growth rates are attributed to the differences between two- and three-dimensional dynamics that are resolved, where transport, dilatation, and production of vorticity by turbulent fluctuations in the third spatial dimension are not present in the two-dimensional DNS (Schilling et al. 2004a). Also, the simulations which included initial velocity conditions (two- and three-dimensional) grew faster than their counterpart two- and three-dimensional DNS with only interfacial perturbations. This is due to the fact that the generation of vertical velocity fluctuations which drive the advection of mass already exist at $\tau=0$ for simulations that include initial velocity perturbations, but are absent in the DNS with only initial interfacial perturbations.

Another measure of the growth of the mixing layer is captured in the definition of an outer-scale or integral-scale Reynolds number, where $R e \equiv L U / \nu$ and $L$ and $U$ 
are integral length- and velocity scales, respectively. However, because the canonical description of this flow includes no boundaries, there are no limits on the size and existence of large-scale eddies. Also, unlike many canonical turbulent flows in which the generation of turbulence is due to a mean component of the flow, Rayleigh-Taylor flows have no mean flow component; however, the generation of large-scale turbulent structures arises from the interaction and coalescence of smaller buoyant structures. Thus, the selection of a length- and velocity scale becomes arbitrary and several definitions have been proposed. First, the release of potential energy can be equated to the formation of turbulent kinetic energy which gives (Snider \& Andrews 1994)

$$
R e^{a}=\sqrt{\frac{g A}{6}} \frac{(2 h)^{3 / 2}}{\nu}
$$

Cook and Dimotakis (2001) proposed that the mixing layer width $h$ and time rateof-change of the mixing layer width $\dot{h}=d h / d t$ adequately represent the length- and velocity scales of the mixing layer, so that

$$
R e^{b}=\frac{h \dot{h}}{\nu}
$$

Another definition proposed by Ramaprabhu and Andrews (2004a) suggest using the terminal velocity of a rising bubble as the velocity scale, where the terminal velocity is defined (Daly 1967; Ratafia 1973)

$$
v_{\infty}=c_{\infty} \sqrt{\frac{\rho_{1}-\rho_{2}}{\rho_{1}} g R_{b}},
$$

where $R_{b}$ is the radius of the bubble and $c_{\infty} \approx 0.7$ is an empirically measured constant. While a statistical measure of the mean bubble radius is infeasible in many experimental configurations, Ramaprabhu and Andrews assumed (in the small Atwood limit) that the mean bubble radius is related to the width of the mixing layer 


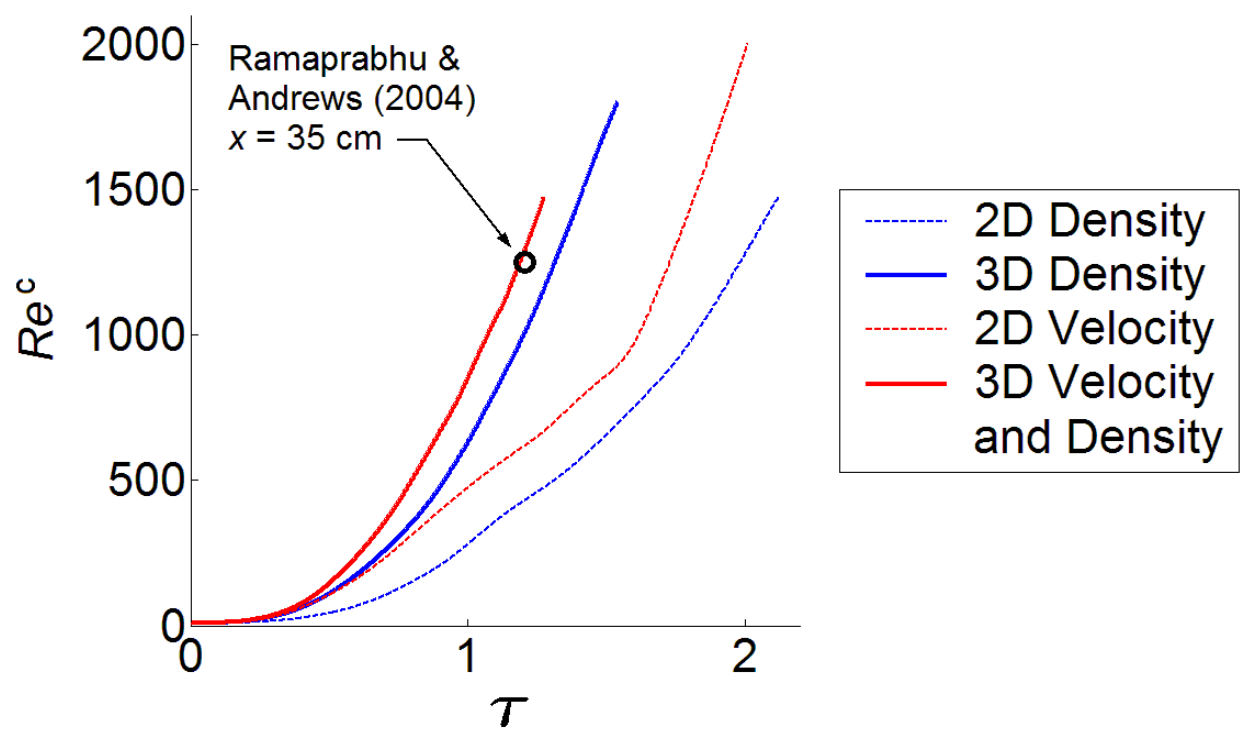

Figure 5.8 Time-evolution of the integral scale Reynolds number $R e^{c}$ based on terminal velocity of a bubble.

width, resulting in

$$
R e^{c}=\frac{1.4 h}{\nu} \sqrt{\frac{A g h}{2}} .
$$

The advantage of this formulation of the $R e^{c}$ is that the only measurement required to calculate the Reynolds number is the mixing layer width. The evolution of $R e^{c}$ for various DNS are plotted in figure 5.8, where a Reynolds number measured by Ramaprabhu and Andrews (2004a) in the water channel is also plotted. It is clear that the three-dimensional DNS with initial velocity and interfacial perturbations provides the closest match to the experimentally-measured Reynolds number at $\tau=1.21$. With the Reynolds number (5.10), it is possible to directly compare the mixing layer widths from the experiment and the DNS at $\tau=1.21$, where the threedimensional DNS show the closest agreement with experimentally-measured values. Figure 5.8 shows the time lag in the development of scales between two- and three- 
dimensional simulations. As discussed in the $§ 5.4$, the two-dimensional simulations with measured initial conditions do not contain the same physics and do not evolve in the same manner as the experiment or the three-dimensional DNS.

\subsection{Small-Scale Results}

A key measure of the small-scale development of the mixing layer is the timeevolution of the molecular mixing fraction $\theta$ at the centerplane of the mixing layer. Figure 5.9 shows the evolution of $\theta(z=0, \tau)$ from various two- and three-dimensional DNS, as well as the same experimentally-measured values shown in $§ 3.1$. All DNS and experiments followed the same qualitative trend of an initial decrease from $\theta \approx$ $0.8-1.0$ to $\theta \approx 0.3-0.45$, followed by an increase at later times. However, there are differences between the experiment and the DNS that deserve discussion. The experiment appears to reach a minimum value of $\theta \approx 0.45$ at $\tau \approx 0.3$, while the simulations do not reach a minimum until later times, $\tau \approx 0.5-0.6$. This transition point, beyond which the rate of molecular mixing is greater that the rate of engulfment of unmixed fluids, is apparently highly sensitive to the initial conditions of the flow and appears to coincide with the transition from a weakly-nonlinear flow to a stronglynonlinear flow, as shown in figures 5.1, 5.2, and 5.5. Further examination is required to understand the differences between the experiment and simulations with respect to this transition point.

To further examine the evolution of the internal structure of the mixing layer, the time-evolution of the two-dimensional density $E_{\rho}\left(k_{2 D}\right)$ and velocity $E_{w}\left(k_{2 D}\right)$ energy spectra at the centerplane of the mixing layer from both two- and three-dimensional DNS are shown in figures 5.10 and 5.11. The two-dimensional spectra were binned into wavenumbers $k_{2 D}=\sqrt{k_{x}^{2}+k_{y}^{2}}$ to produce one-dimensional spectra in wavenumber 


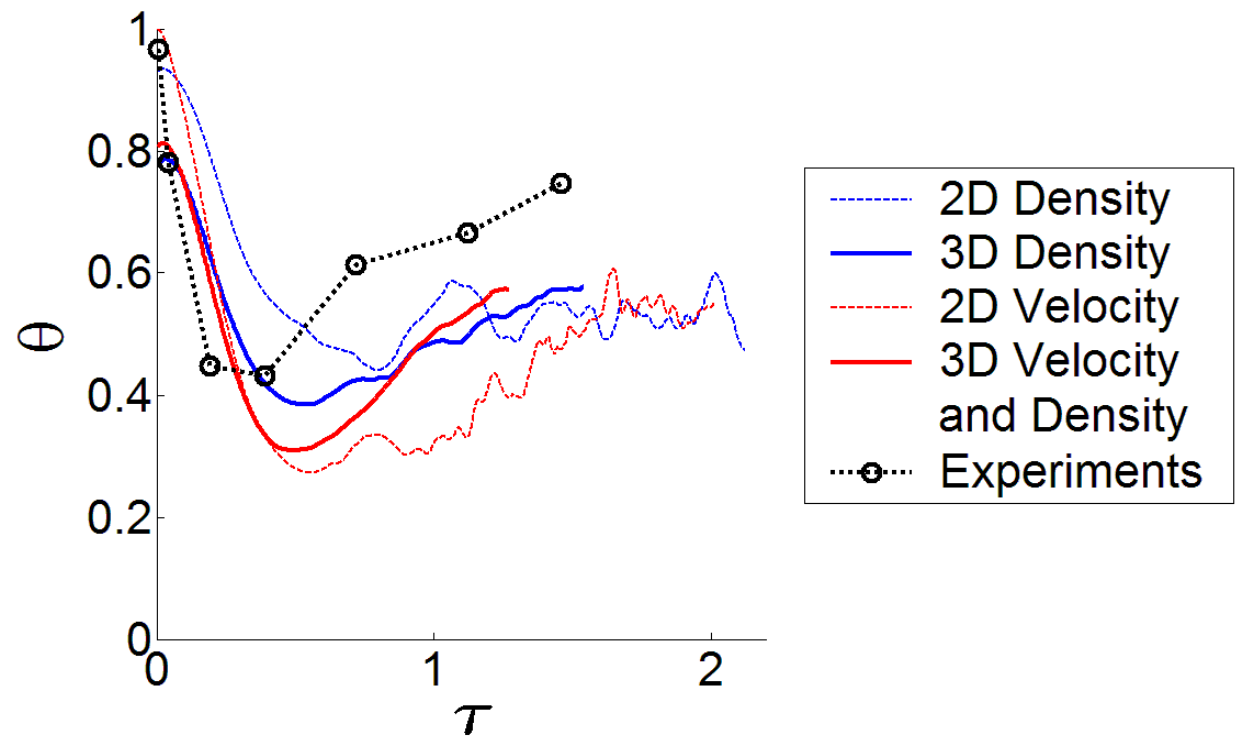

Figure 5.9 Time-evolution of the molecular mixing fraction $\theta$ at the centerplane from various DNS and experimentally-measured values.

space. Compensated spectra from both two- and three-dimensional DNS are shown in figures 5.12 and 5.13, respectively. Both two- and three-dimensional simulations exhibit an inertial range scaling of $k^{n}$ where $n>-5 / 3$ for the density fluctuations at later times; however, no significant inertial range scaling is found for the velocity fluctuations. A similar difference between the slope of the inertial range of $E_{\rho}(k)$ and a $k^{-5 / 3}$ scaling was found by Cook et al. (2004) using high-resolution LES. The existence of an inertial range for density fluctuations, while no such scaling is apparent in the velocity fluctuations, coincides with the difference in the centerplane density and velocity fields shown in figure 5.5. It is believed that because $S c>1$, density fluctuations persist at higher wavenumbers than the velocity fluctuations, allowing for the earlier formation of an inertial range.

In the same manner that the probability density functions of the centerplane density values are related to the measure of molecular mixing $\theta$, such a relationship 

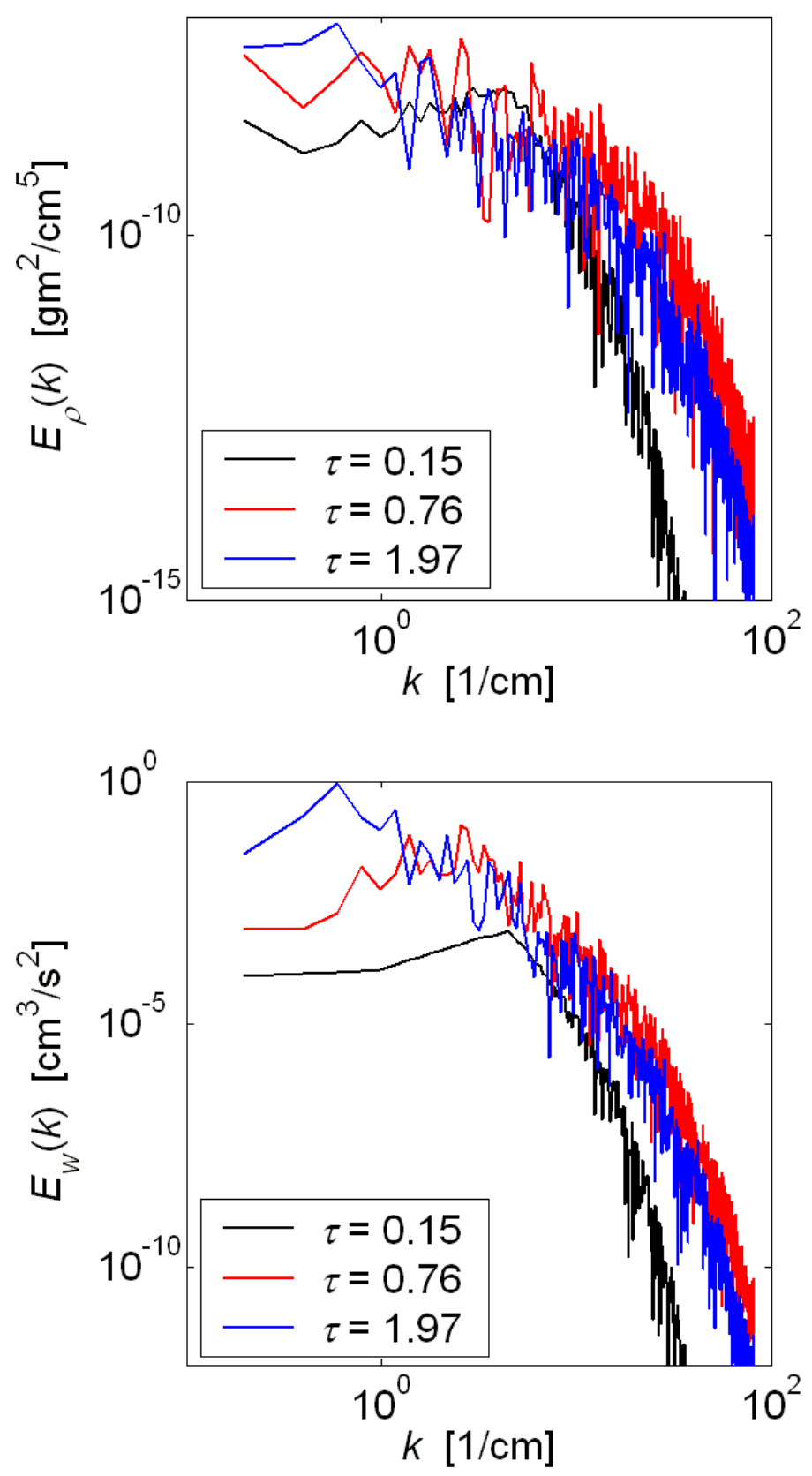

Figure 5.10 Time-evolution of $E_{\rho}\left(k_{2 D}\right)$ (top) and $E_{w}\left(k_{2 D}\right)$ (bottom) from a two-dimensional DNS with initial velocity perturbations. 

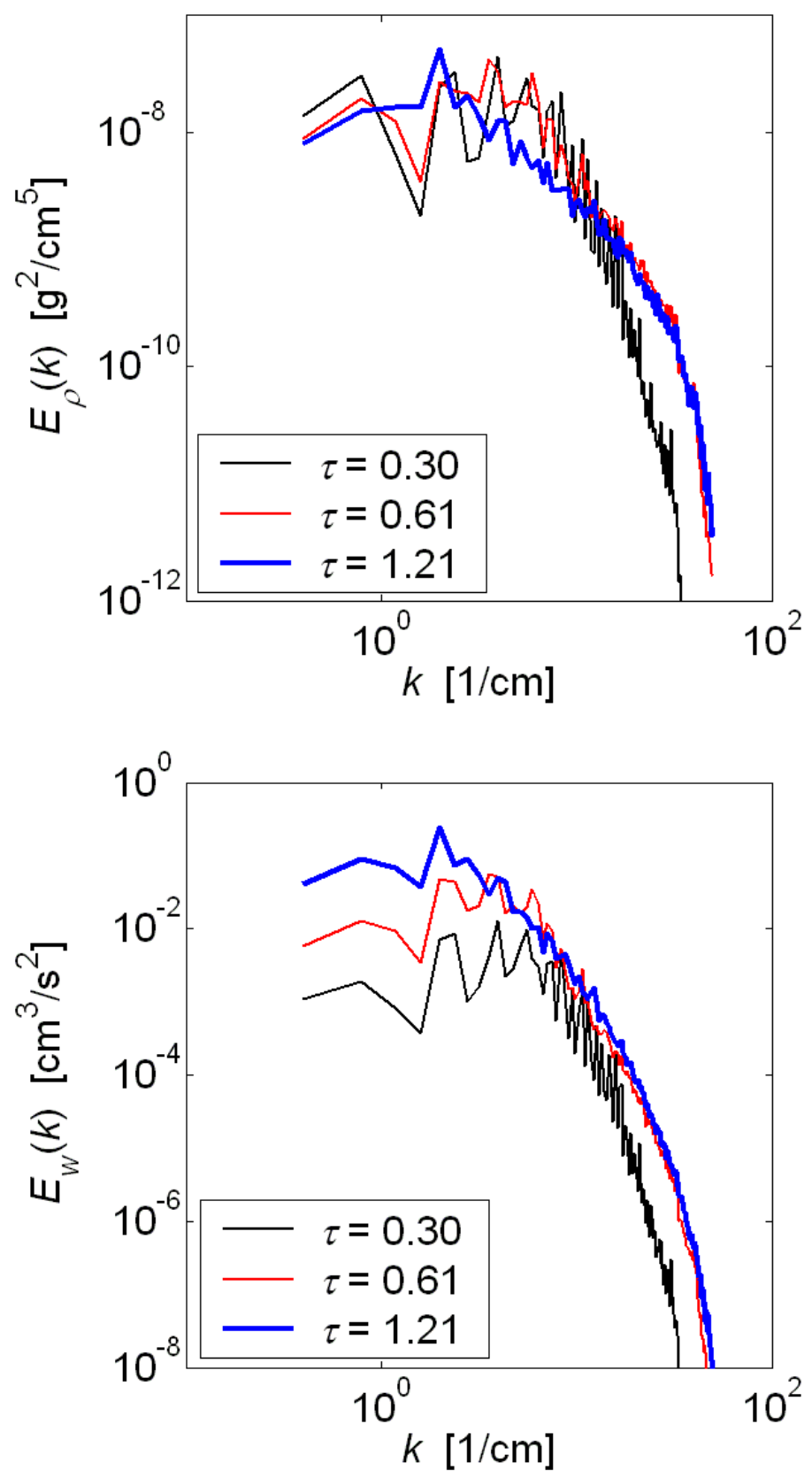

Figure 5.11 Time-evolution of $E_{\rho}\left(k_{2 D}\right)$ (top)and $E_{w}\left(k_{2 D}\right)$ (bottom) from a three-dimensional DNS with initial velocity and interfacial perturbations. 

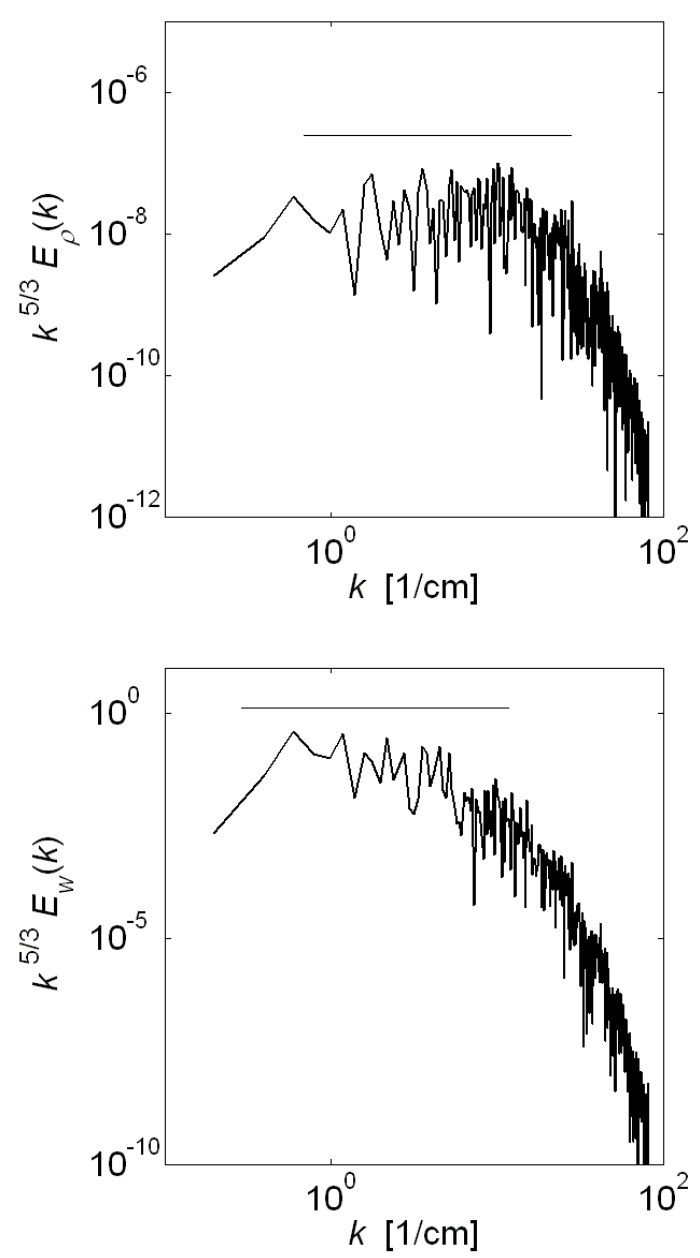

Figure 5.12 Compensated energy spectra showing the lack of a $k^{-5 / 3}$ inertial range scaling for $E_{\rho}\left(k_{2 D}\right)$ (top) and $E_{w}\left(k_{2 D}\right)$ (bottom) from a two-dimensional DNS with initial velocity perturbations. 

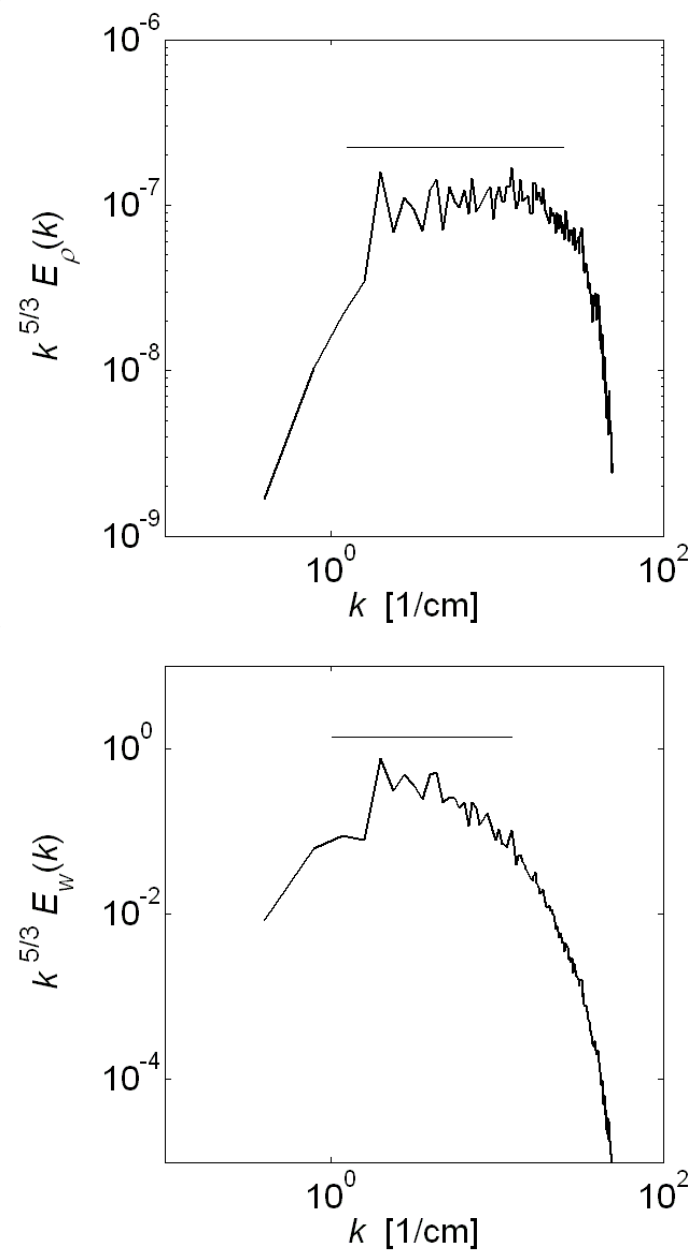

Figure 5.13 Compensated energy spectra showing the lack of a $k^{-5 / 3}$ inertial range scaling for $E_{\rho}\left(k_{2 D}\right)$ (top) and $E_{w}\left(k_{2 D}\right)$ (bottom) from a three-dimensional DNS with initial velocity and interfacial perturbations. 
also exists between the fluctuating density energy spectra and $\theta$. From the definition of $\theta, B_{2}=0.25$ at the centerplane of a Rayleigh-Taylor mixing layer, resulting in $\theta=1-4 B_{0}$. This equation can be simplified further by noting that the integral of the density energy spectrum over all wavenumbers yields the variance of density fluctuations, similar to equation (3.2). Thus, $\theta$ is linked to the evolution of the fluctuating density spectrum by

$$
\begin{aligned}
\theta(\tau) & =1-4 \sqrt{\left\langle\rho^{2}\right\rangle(\tau)} \\
& =1-4 \sqrt{\int_{k_{\min }}^{k_{\max }} E_{\rho}(k, \tau) d k}
\end{aligned}
$$

It is shown in the evolution of the fluctuating density spectra that, as energy develops in the smallest wavenumbers at late time, the integral of density spectrum decreases in value, corresponding to an increase in $\theta$.

Direct comparisons of the two-dimensional spectra from the simulations and the experimentally-measured spectra are not feasible because the experimentallymeasured spectra are one-dimensional in wavenumber space $\mathbf{k}=k_{x} \hat{\mathbf{e}}_{x}$. However, the spectra shown in figures 5.10 and 5.11 were calculated in two-dimensional wavenumber space $\mathbf{k}=k_{x} \hat{\mathbf{e}}_{x}+k_{y} \hat{\mathbf{e}}_{y}$ and separated into one-dimensional wavenumber bins, assuming isotropy in the homogeneous directions. However, this assumption of isotropy is not valid for these simulations, especially at the early times. While a tendency towards isotropy is shown in the later stages of the centerplane density and velocity fields in figure 5.5, no quantitative measure of the anisotropy has been made. Also, Tennekes and Lumley (1972) and Pope (2000) both point out that spectral measurements made in one-dimensional wavenumber space are biased when the physical fluctuations exist in two- or three-dimensional space. Thus, for comparison purposes, one-dimensional spectra will also be computed from the simulation data, where the phase-averaged 
one-dimensional spectrum is defined

$$
E_{\phi}(k)=\frac{1}{N_{y}} \sum_{j=1}^{N y} E_{\phi}(k, j),
$$

where $\phi$ is any scalar quantity and $j$ is the row of grid points along which a onedimensional spectrum $E_{\phi}(k)$ has been calculated. Figures 5.14 and 5.15 show the phase-averaged, one-dimensional spectra for the same two- and three-dimensional DNS shown in figures 5.10 and 5.11. For both figures, the numerical spectra have been superimposed on top of the experimentally-measured density spectra from the same development time $\tau$.

The density spectra from the two-dimensional DNS with initial velocity perturbations exhibit very good agreement with the experimentally-measured density spectra at $\tau=0.39,0.72,1.12$, with the only discrepancy occurring at the lowest wavenumbers. This is explained by the lack of statistical convergence in the lower wavenumbers of the density and velocity spectra, limited by the domain width of the simulation $L_{x}=32 \mathrm{~cm}$. This distance is small when compared to the experimental measurements which were taken over a distance of $L \approx 250 \mathrm{~cm}$, converting time to space by Taylor's hypothesis. Comparisons between the three-dimensional DNS and the experimentally-measured density spectra show similar results, with one exception. The spectral cascade between the density fluctuations at the highest wavenumbers in the three-dimensional DNS occurs at a slower rate than in the experiment, where $E_{\rho}(k) \sim k^{n}, n>-3$. It is hypothesized that this occurs because of the limited grid resolution applied to the numerical domain which resulted in the introduction of aliasing errors into the domain seen in the up-turn of the spectra at the highest wavenumbers. Filtering of the DNS was necessary to mitigate these errors. Also, the limited resolution of the three-dimensional DNS affects the spectral resolution of the 

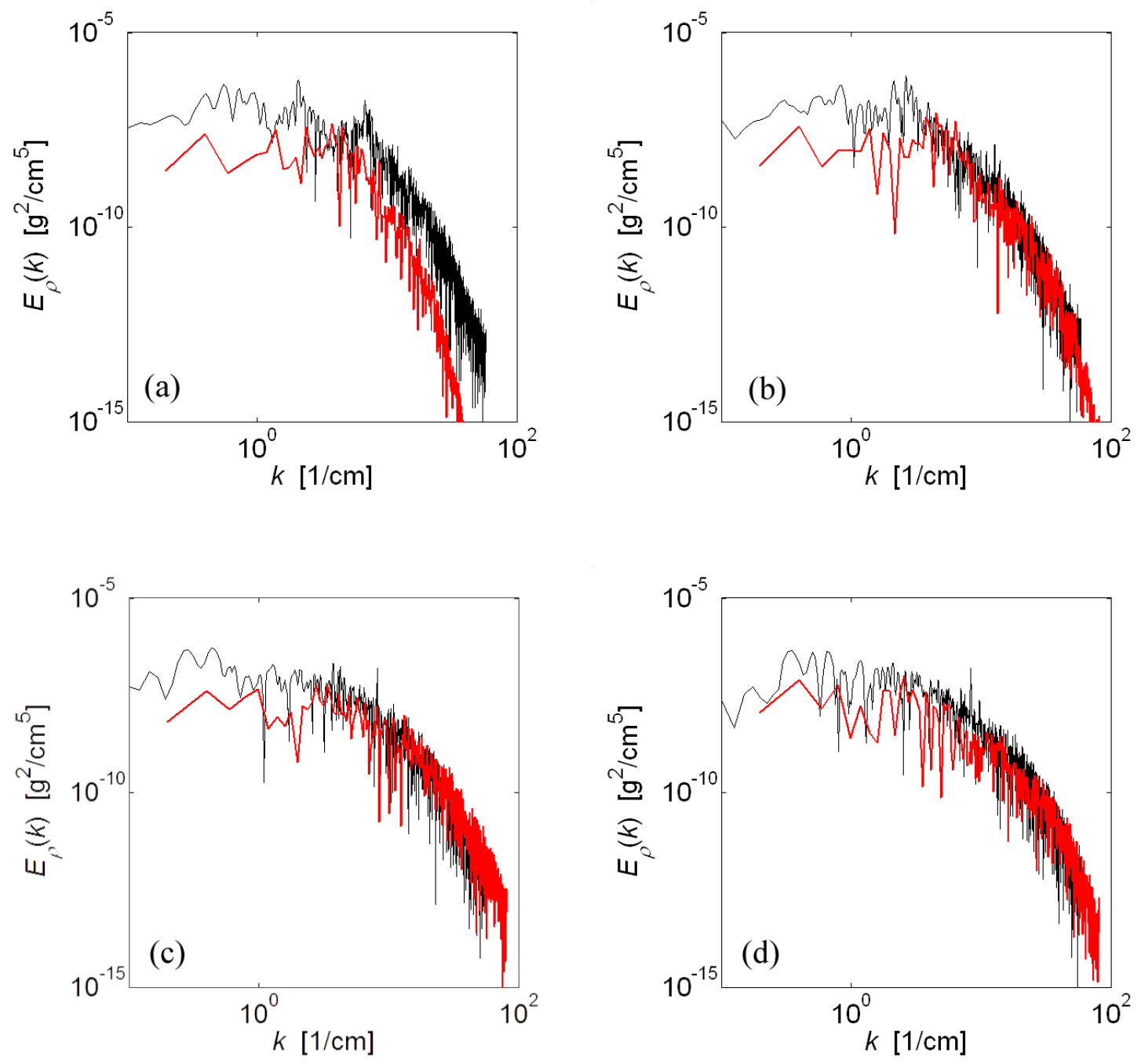

\section{Experiment \\ 2D Simulation}

Figure 5.14 Time-evolution of the density spectrum from a two-dimensional DNS with initial velocity perturbations at: (a) $\tau=0.19$; (b) $\tau=0.39$; (c) $\tau=0.72$, and; (d) $\tau=1.12$. Experimentally-measured density spectra at equivalent times are shown in black. 

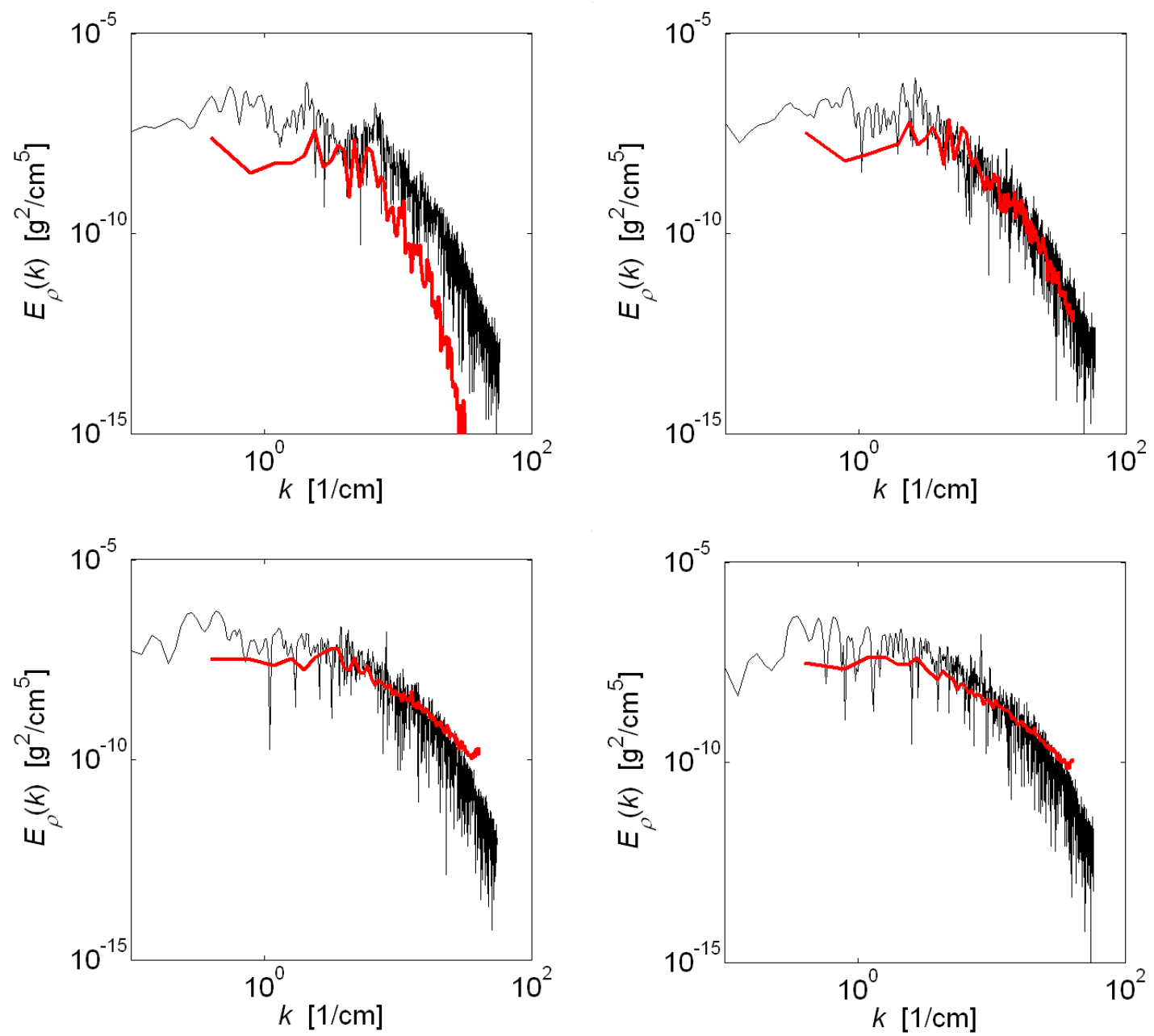

\section{Experiment} 3D Simulation

Figure 5.15 Time-evolution of the density spectrum from a three-dimensional DNS with initial velocity and interfacial perturbations at: (a) $\tau=0.19$; (b) $\tau=0.39 ;$ (c) $\tau=0.72$, and; (d) $\tau=1.12$. Experimentally-measured density spectra at corresponding times are shown in black. 
higher modes, where all wavenumbers $k>12.6$ are supported on 4 grid points per wavelength, or less. The exact results of the numerical filtering, aliasing errors, and lack of spectral resolution in the higher wavenumbers of the spectra are not exactly known, but should be assessed with higher-resolution simulations.

Also, the phase-averaged density spectra from the three-dimensional DNS appear to contain less noise content than the two-dimensional DNS and the experiments. This is attributed to a greater degree of averaging that is performed in the calculation of the density spectra from the three-dimensional simulation, eliminating statistical noise. Such an averaging procedure is not capable of being performed in the experiment and two-dimensional DNS and experiments because density information in the spanwise direction is either not known or constrained by the simulation. 


\section{CONCLUSIONS}

\subsection{Experimental Conclusions}

In this investigation, the initial velocity and density fluctuations present at the trailing edge of the splitter plate in a statistically-stationary, small Atwood number, Rayleigh-Taylor mixing layer experiment have been measured. Initial density fluctuations in the streamwise $(x)$ direction were measured using new high-resolution, E-type thermocouples with a weld bead diameter of $0.16 \mathrm{~mm}$. The new thermocouples were also used to measure the energy spectra of density fluctuations and the degree of molecular mixing at the centerplane of the mixing layer for several stages of development. The initial velocity fluctuations $\left(u^{\prime}\right.$ and $\left.w^{\prime}\right)$ in the near wake $(x=0.5 \mathrm{~cm})$ of the splitter plate were measured with PIV in the water channel using a non-buoyant configuration. Two-fluid interfacial perturbations were measured in the transverse (y) direction using PLIF. The summation of the three measurements performed constitute the first complete, three-dimensional measurement of the initial conditions of a Rayleigh-Taylor unstable interface. Conclusions stemming from this work are listed below.

- The initial interfacial perturbation was shown to contain a broad-banded perturbation in wavenumber space with a steep drop-off at the largest wavenumbers. The peak perturbation at $x=0.1 \mathrm{~cm}$ downstream from the splitter plate was at $k \approx 11 \mathrm{~cm}^{-1}$, which is one order of magnitude greater in amplitude than all of the other perturbations present at $x=0.1 \mathrm{~cm}$. It is speculated that these perturbations are imparted on the two-fluid interface by the splitter plate and end-screen combination.

- The spectrum of initial velocity fluctuations due to the wake shedding effect behind the splitter plate has been measured. The momentum deficit in the 
wake of the splitter plate was shown to be minimal, where $u_{\text {def }} / U_{m}=0.05$.

- The two-fluid interfacial perturbation in the spanwise $(y)$ direction has been measured using off-axis imaging techniques and characterized by an interfacial perturbation spectrum $E_{\zeta}(k)$. Velocity perturbations in the spanwise direction were found to be negligible.

- The evolution of the molecular mixing fraction at the centerplane $\theta(z=0, \tau)$ was measured using new, higher resolution thermocouples. It was found that $\theta$ decreased to a minimum value at $\tau \approx 0.3$, similar to the findings of previous researchers. However, the minimum value of $\theta$ was found to be smaller than previously measured by Wilson (2002) and Ramaprabhu and Andrews (2004a), where a minimum of $\theta \approx 0.45$ was reached.

\subsection{Numerical Conclusions}

The measurements of initial interfacial and velocity perturbations were used to parameterize the initial conditions of two- and three-dimensional DNS of the water channel experiment. These simulations are the first DNS of the water channel experiment. Major contributions and conclusions are highlighted below.

- Methods for implementing fully-measured, fully-anisotropic interfacial and velocity perturbations of a Rayleigh-Taylor flow have been demonstrated in DNS of the water channel experiment. All initial perturbations have been based on measured, one-dimensional energy spectra, $E_{\zeta}(k)$ and $E_{w}(k)$, with no ad hoc perturbations.

- DNS using measured initial velocity perturbations in the streamwise direction and interfacial perturbations in the spanwise direction exhibit the clos- 
est match to the large- and small-scale results measured from the experiment. This contributes to the hypothesis that Rayleigh-Taylor experiments are controlled initially by the deposition of momentum at the two fluid interface, rather that the deposition of baroclinic vorticity at a stationary, perturbed interface (Ramaprabhu \& Andrews 2004b; Schilling et al. 2004b).

- A transition in the degree of mixedness at the centerplane of the mixing layer has been shown, such that $\theta$ decreases until this point is reached and increases thereafter. Empirical and numerical observations suggest that this transition is related to the saturation of the initial perturbations and to the onset of KelvinHelmholtz instabilities. Therefore, this transition point in time and value of $\theta$ is very sensitive to the initial conditions.

- The spectral cascade of density variance develops an inertial range scaling of not quite $k^{-5 / 3}$ over one decade of wavenumbers, similar to the inertial range scaling of $E_{\rho}(k)$ found by Cook et al. (2004) using high resolution LES. No such range is apparent in the vertical velocity fluctuations. The formation of a spectral cascade power-law in $E_{\rho}(k)$ before an inertial range scaling is noticed in $E_{w}(k)$ is presumably due to the difference in mass and momentum diffusivities, where $S c=7$.

- Three-dimensional DNS of the water channel exhibited a faster mixing layer growth rate than two-dimensional DNS with analogous initial conditions for the time span occurring in the experiment.

\subsection{Future Work}

The work conducted in this investigation can be extended in several directions: 
- Experimentally, it would be advantageous to extend the measurement principles presented in this work to higher Atwood number, gas-phase experiments. This would directly relate the experimental investigation of higher Atwood number flows and the initialization of higher Atwood number, three-dimensional numerical simulations.

- Another experimental extension of this work would be to use the same water channel to study the effects of mass diffusivity upon the growth and internal development of a Rayleigh-Taylor mixing layer. This could be accomplished through the use of fresh and salt water to create the density difference between the streams, resulting in $S c \sim 1000$.

- The water channel arrangement could be run in a variable density arrangement, where the two fluids are chemically reacting. In such an experiment, the degree of molecular mixing could be measured by the chemically reacting indicators, such as acidity indicators. This experiment would then allow for a more global measurement of the amount of mixing across the mixing layer width.

- The parameterization of the initial conditions in the streamwise direction can be enhanced to include both the effects of the initial interfacial perturbations and initial velocity perturbations. While it is believed that the initial velocity perturbations are the primary driver of the two-fluid interface as $\tau \rightarrow 0$, the inclusion of the interfacial spectrum measured at $\tau=0.004$ would include the perturbations at $k \approx 11 \mathrm{~cm}^{-1}$ that are imparted by the end-screen. Perhaps this interfacial perturbation, coupled with the initial velocity perturbation and spanwise interfacial perturbation, is responsible for the transition to a stronglynonlinear flow at $\tau<0.5$. 
- With respect to the lower Atwood number simulations presented in this work, it is desirable to increase the spatial resolution of the three-dimensional DNS, so that the initial conditions and fluctuating density and velocity fields are better resolved.

- One of the motivations driving this research is the goal of modeling the turbulent Rayleigh-Taylor mixing process with respect to the initial perturbations of the flow. In this respect, it is useful to further analyze the DNS data to provide insight into the internal structure of the turbulence and mixing processes by examining the evolution of correlations between the velocity and density fields. Also, the initialization of the turbulence and mixing models can be accomplished by examining the early-time effects of initial conditions on the transition to a strongly-nonlinear flow.

- Concerning the subject of turbulence and mixing model development, the examination of the differences from two- and three-dimensional DNS could provide insight into the development of models that simulate three-dimensional physical effects within two-dimensional simulations (Schilling 2004). The benefit to this research lies in the ability to perform economical, two-dimensional simulations of more complicated physical configurations in the pursuit of experimental design goals rather than the elucidation of the fundamental hydrodynamic processes.

- One interesting aspect of this work is that it reports on the results of threedimensional DNS which were initialized with fully-anisotropic perturbations. While a tendency towards isotropy was observed at later times, no formal examination of the anisotropy of the density field or rate-of-return to isotropy has been performed. Such a measure could prove to be insightful with respect to the effects of initial conditions on the development of a three-dimensional mixing 
layer. 


\section{REFERENCES}

Adrian, R. J. 1991 Particle-imaging techniques for experimental fluid mechanics. Ann. Rev. Fluid Mech. 23, 261-304.

Adrian, R. J. 1997 Dynamic ranges of velocity and spatial resolution of particle image velocimetry. Meas. Sci. Technol. 8, 1393-1398.

Andrews, M. J. 1986 Turbulent mixing by Rayleigh-Taylor instability. Ph.D. thesis. Imperial College of Science and Technology, London.

Andrews, M. J. \& Spalding, D. B. 1990 A simple experiment to investigate two-dimensional mixing by Rayleigh-Taylor instability. Phys. Fluids A 2, 922-927.

Anuchina, N. N., Kucherenko, Y. A., Neuvazhaev, V. E., Ogibina, V. N., Shibarshov, L. I. \& Yakovlev, V. G. 1978 Turbulent mixing at an accelerating interface between liquids of different densities. Izv. Akad Nauk. SSSR, Mekh. Zhidk. Gaza 6, 157-160.

Arroyo, M. P. \& Greated, C. A. 1991 Stereoscopic particle image velocimetry. Meas. Sci. Technol. 2, 1181-1186.

Atzeni, S. \& Meyer-ter-Vehn, J. 2004 The Physics of Inertial Fusion: Beam Plasma Interaction, Hydrodynamics, Hot Dense Matter, International Series of Monographs on Physics. Oxford University Press.

Betti, R., Umansky, M., Lobatchev, V., Goncharov, V. N. \& McCrory, R. L. 2001 Hotspot dynamics and deceleration-phase Rayleigh-Taylor instability of imploding inertial confinement fusion capsules. Phys. Plasmas 8, 5257-5267.

Blackman, R. B. \& Tukey, J. W. 1959 Particular pairs of windows. The Measurement of Power Spectra, From the Point of View of Communications Engineering. Dover.

Boris, J. P., Grinstein, F. F., Oran, E. S. \& Kolbe, R. L. 1992 New insights into large eddy simulation. Fluid Dyn. Res. 10, 199-228.

Broadwell, J. E. \& Briedenthal, R. E. 1982 A simple model of mixing and chemical reaction in a turbulent shear layer. J. Fluid Mech. 125, 397-410.

Cabot, W. H., Schilling, O. \& Zhou, Y. 2004 Influence of subgrid scales on resolvable turbulence and mixing in Rayleigh-Taylor flow. Phys. Fluids 16, 495-508.

Canny, J. 1986 A Computational Approach to Edge Detection. IEEE Transactions on Pattern Analysis and Machine Intelligence PAMI-8, 679-698.

Chandrasekhar, S. 1961 Hydrodynamic and Hydromagnetic Stability. Dover.

Cook, A. W., Cabot, W. \& Miller, P. L. 2004 The mixing transition in Rayleigh-Taylor instability. J. Fluid Mech. 511, 333-362.

Cook, A. W. \& Dimotakis, P. E. 2001 Transition stages of Rayleigh-Taylor instability between miscible fluids. J. Fluid Mech. 443, 69-99.

Cook, A. W. \& Dimotakis, P. E. 2002 Corrigendum. J. Fluid Mech. 457, 410-411.

Cook, A. W. \& Zhou, Y. 2002 Energy transfer in Rayleigh-Taylor instability. Phys. Rev. E 66, 026312.

Cui, A. Q. \& Street, R. L. 2004 Large-eddy simulation of coastal upwelling flow. Envir. Fluid Mech. 4, 197-223.

Dai, Z., Tseng, L. K. \& Faeth, G. M. 1994 Structure of round, fully developed, buoyant turbulent plumes. J. Heat Transfer 116, 409-417. 
Daly, B. J. 1967 Numerical study of two-fluid Rayleigh-Taylor instability. Phys. Fluids 10, 297-307.

Dalziel, S. B., Linden, P. F. \& Youngs, D. L. 1999 Self-similarity and internal structure of turbulence induced by Rayleigh-Taylor instability. J. Fluid Mech. 399, 1-48.

Dankwerts, P. V. 1952 The definition and measurement of some characteristics of mixtures. Appl. Sci. Res. 3, 279-296.

Dimonte, G. \& Schneider M. 1996 Turbulent Rayleigh-Taylor instability experiments with variable acceleration. Phys. Rev. E 54, 3740-3743.

Dimonte, G., Youngs, D. L., Dimits, A., Weber, S., Marinak, M., Wunsch, S., Garasi, C., Robinson, A., Andrews, M. J., Ramaprabhu, P., Calder, A. C., Fryxell, B., Biello, J., Dursi, L., MacNeice, P., Olson, K., Ricker, P., Rosner, R., Timmes, H., Tufo, H., Young, Y.-N., \& Zingale, M. 2004 A comparative study of the turbulent RayleighTaylor (RT) instability using high-resolution 3D numerical simulations: The AlphaGroup collaboration. Phys. Fluids 16, 1668-1693.

Dimotakis, P. E. 2000 The mixing transition in turbulent flows. J. Fluid Mech. 409, 69-98.

Drazin, P. G. \& Reid, W. H. 2004 Hydrodynamic Stability. 2nd Ed. Cambridge University Press.

Emmons, H. W., Chang, C. T. \& Watson, B. C. 1960 Taylor instability of finite surface waves. J. Fluid Mech. 7, 177-193.

Fisher, C. E. \& Ball, K. S. 1999 Plume dynamics in natural convection in a horizontal cylindrical annulus. J. Heat Transfer 121, 598-602.

Grue, J., Jensen, A., Rusas, P., \& Sveen, J. K. 2000 Breaking and broadening of internal waves. J. Fluid Mech. 413, 181-217.

Haan, S. W. 1989 Onset of nonlinear saturation for Rayleigh-Taylor growth in the presence of a full spectrum of modes. Phys. Rev. A 39, 5812-5825.

Haan, S. W., Pollaine, S. M., Lindl, J. D., Suter, L. J., Berger, R. L., Power, L. V., Alley, W. E., Amendt, P. A., Futterman, J. A., Levedahl, W. K., Rosen, M. D., Rowley, D. P., Sacks, R. A., Shestakov, A. I., Strobel, G. L., Tabak, M., Weber, S. V., Zimmerman, G. B. \& Krauser, W. J. 1995 Design and modeling of ignition targets for the National Ignition Facility. Phys. Plasmas 2, 2480-2487.

Jacobs, J. W. 1992 Shock-induced mixing of a light-gas cylinder. J. Fluid Mech. 234, 629 649.

Joseph, D. D. 1990 Fluid dynamics of two miscible liquids with diffusion and gradient stress. Eur. J. Mech. B/Fluids 9, 565-596.

Kent, J. C. \& Eaton, A. R. 1982 Stereo photography of neutral density He-filled bubbles for 3-D fluid motion studies in an engine cylinder. Appl. Opt. 21, 904-912.

Kilkenny, J. D., Glendinning, S. G., Haan, S. W., Hammel, B. A., Lindl, J. D., Munro, D., Remington, B. A., Weber, S. V., Knauer, J. P. \& Verdon, C. P. 1994 A review of the ablative stabilization of the Rayleigh-Taylor instability in regimes relevant to inertial confinement fusion. Phys. Plasmas 1, 1379-1389.

Kraft, W. N. 2004 Experimental investigation of a stratified buoyant wake. M.S. thesis. Texas A\&M University.

Kukulka, D. J. 1981 Thermodynamic and transport properties of pure and saline water. M.S. thesis, State University of New York at Buffalo. 
Lee, M. P., McMillin, B. K., Palmer, J. L. \& Hanson, R. K. 1992 Planar Fluorescence imaging of a transverse jet in a supersonic cross-flow. J. Prop. Power 8, 729-735.

Leslie, D. C. \& Quarini G. L. 1979 The application of turbulence theory to the formulation of subgrid modelling procedures. J. Fluid Mech. 91, 65-91.

Lilly D. K. 1967 The representation of small-scale turbulence in numerical simulation experiments. Proc. IBM Scientific Computing Symp. on Environmental Sciences 195-210.

Linden P. F. \& Redondo, J. M. 1991 Molecular mixing in Rayleigh-Taylor instability. Part I: Global mixing. Phys. Fluids A 3, 1269-1277.

Linden P. F., Redondo, J. M. \& Youngs D. L. 1994 Molecular mixing in Rayleigh-Taylor instability. J. Fluid Mech. 265, 97-124.

Lindl, J. D. 1998 Inertial Confinement Fusion: The Quest for Ignition and Energy Gain Using Indirect Drive. AIP Press.

Marmottant, P. H. \& Villermaux, E. 2004 On spray formation. J. Fluid Mech. 498, 73-111.

Mills, A. F. 1999 Heat Transfer. Prentice Hall.

Molchanov, O. A. 2003 On the origin of low- and middler-latitude ionospheric turbulence. Phys. Chem. Earth 29, 559-567.

Mueschke, N. J. \& Andrews, M. J. 2004 Investigation of scalar measurement error in diffusion processes. International Mechanical Engineering Congress and RDESD Expo. Anaheim, CA.

Papanicolaou P. N. \& List E. J. 1987 Statistical and spectral properties of tracer concentration in round buoyant jets. Int. J. Heat Mass Transf. 30, 2059-2071.

Pope, S. B. 2000 Turbulent Flows. Cambridge University Press.

Pratt W. K. 1991 Digital Image Processing 2nd Ed. Wiley-Interscience.

Ramaprabhu, P. 2003 On the dynamics of Rayleigh-Taylor mixing. Ph.D. dissertation. Texas A\&M University.

Ramaprabhu, P. \& Andrews, M. 2003 Simultaneous measurements of velocity and density in buoyancy-driven mixing. Exp. Fluids 34, 98-106.

Ramaprabhu, P. \& Andrews, M. J. 2004a Experimental investigation of Rayleigh-Taylor mixing at small Atwood numbers. J. Fluid Mech. 502, 233-271.

Ramaprabhu, P. \& Andrews, M. J. 2004b On the initialization of Rayleigh-Taylor simulations. Phys. Fluids 16, L59-L62.

Ratafia, M. 1973 Experimental investigation of turbulent mixing by Rayleigh-Taylor instability. Phys. Fluids 16, 1207-1210.

Rayleigh, L. 1884 Investigation of the equilibrium of an incompressible heavy fluid of variable density. Proc. London Math. Soc. 14, 170-177.

Read, K. I. 1984 Experimental investigation of turbulent mixing by Rayleigh-Taylor instability. Physica D 12, 45-58.

Ristorcelli J. R. \& Clark T. T. 2004 Rayleigh-Taylor turbulence: self-similar analysis and direct numerical simulations. J. Fluid Mech. 507, 213-253.

Sandoval, D. L. 1995 The dynamics of variable density turbulence. Ph.D. thesis. University of Washington.

Schilling, O. 2004 Lawrence Livermore National Laboratory, Livermore, CA. Private communication. 
Schilling, O., Latini, M. \& Don, W.-S. 2004a Investigation of the large-scale and statistical properties of Richtmyer-Meshkov instability-induced mixing. Proceedings of the Ninth International Workshop on the Physics of Compressible Turbulent Mixing. Cambridge, UK.

Schilling, O., Mueschke, N. \& Andrews, M. 2004b Direct numerical simulations of miscible, small Atwood number Rayleigh-Taylor instability-induced mixing. Proceedings of the Ninth International Workshop on the Physics of Compressible Turbulent Mixing. Cambridge, UK.

Sharp, D. H. 1984 An overview of Rayleigh-Taylor instability. Physica D 12, 3-18.

Smagorinsky, J. 1963 General circulation experiments with the primitive equations: I. The basic equations. Mon. Weather Rev. 91, 99-164.

Smarr, L., Wilson, J. R., Barton, R. T. \& Bowers, R. L. 1981 Rayleigh-Taylor overturn in super-nova core collapse. Astrophysical J. 246, 515-525.

Snider, D. M. \& Andrews, M. J. 1994 Rayleigh-Taylor and shear driven mixing with an unstable thermal stratification. Phys. Fluids A 6, 3324-3334.

Sveen, J. K. 2004 An introduction to MatPIV v. 1.6.1. eprint series, Mech. and Appl. Math. $\mathbf{2}, 1-27$.

Taylor, G. I. 1938 The spectrum of turbulence. Proc. Royal Soc. London Ser. A 164, 476490.

Taylor, G. I. 1950 The instability of liquid surfaces when accelerated in a direction perpendicular to their planes. Proc. Royal Soc. London 201, 192-196.

Tennekes, H. \& Lumley, J. L. 1972 A First Course in Turbulence. MIT Press.

Thomas, G. O. 2003 The aerodynamic breakup of ligaments. Atom. Sprays 13, 117-129.

van Oord, J. 1997 The design of a stereoscopic DPIV-system. Delft University of Technology Report MEAH-161.

Willert, C. 1997 Stereoscopic digital particle image velocimetry for application in wind tunnel flows. Meas. Sci. Technol. 8, 1465-1479.

Wilson, P. N. 2002 A study of buoyancy and shear driven turbulence within a closed water channel. Ph.D. dissertation. Texas A\&M University.

Wilson, P. N. \& Andrews, M. J. 2002 Spectral measurements of Rayleigh-Taylor mixing at low-Atwood number. Phys. Fluids A 14, 938-945.

Youngs, D. L. 1984 Numerical simulations of turbulent mixing by Rayleigh-Taylor instability. Physica D 12, 32-44.

Youngs, D. L. 1991 Three-dimensional numerical simulations of turbulent mixing by Rayleigh-Taylor instability. Phys. Fluids A 3, 1312-1320.

Zandt, G., Gilbert, H., Owens, T. J., Ducea, M., Saleeby, J. \& Jones, C. H. 2004 Active foundering of a continental arc root beneath the southern Sierra Nevada in California. Nature 431, 41-46. 


\section{APPENDIX A}

\section{SCALAR MEASUREMENT ERROR IN DIFFUSION AND MIXING PROCESSES}

1

In variable-density, multi-fluid and reacting flows, a quantitative measure of the degree of molecular mixing is crucial to the development of turbulent transport and mixing models. Characterization of Rayleigh-Taylor instability-induced mixing and other mixing processes requires scalar measurement devices with an adequate probe volume size. Spatial-averaging, which occurs due to the finite probe volume size, can lead to errors in resolving the density or scalar gradients between pockets of unmixed fluids. Given a probe volume size and a priori knowledge of the functional profile of the diffusion layer being measured, it is possible to estimate the measurement error due to spatial-averaging that has occurred and make corrections accordingly.

Assume that two fluids are separated by an interface, but are not mixed at $t=0$. Any perturbation on that interface will produce an interface as seen in figure A.1 and a scalar trace at the centerline similar to the unmixed case (solid line) shown in figure A.2. The evolution of miscible fluids or scalars is controlled by the growth of the initial perturbation by external forces and by the diffusion of the scalar values at the interface. In the single-mode case, the two parameters required to analytically evaluate $\theta$ are the wavelength of the interfacial perturbation, $\lambda$, and the width of the interfacial diffusion layer, $L$.

In this investigation, it is of interest to understand how $B_{0}$ and $\theta$ change with respect to $L / \lambda$, the ratio of the diffusion thickness to the wavelength of the perturbation. Over a range of $L / \lambda$ values, it is possible to evaluate $B_{0}, B_{2}$, and $\theta$ as a function

\footnotetext{
${ }^{1}$ Portions of this section, including all figures, have been reprinted with permission from Mueschke \& Andrews (2004). Copyright ASME 2004.
} 


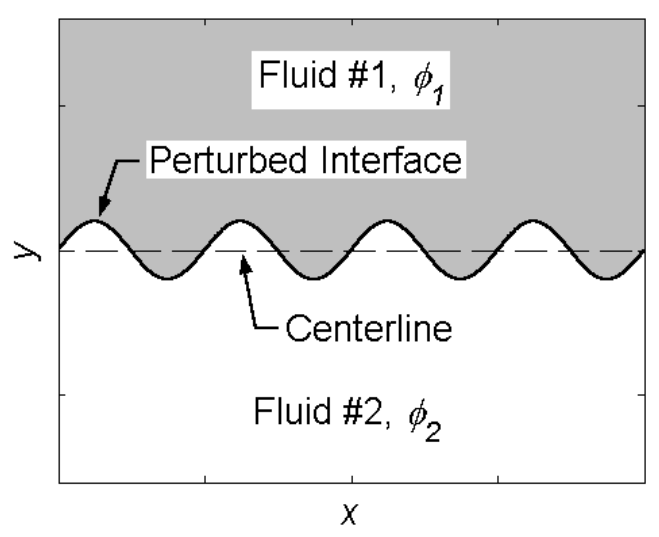

Figure A.1 Schematic of a perturbed interface between two fluids or scalars.

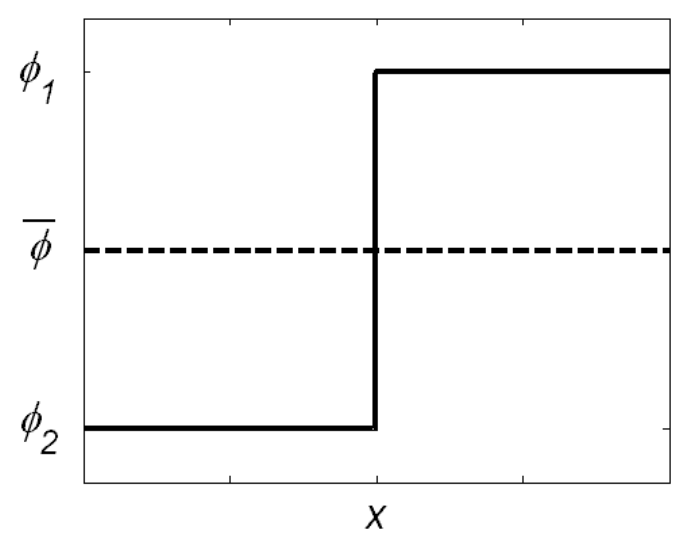

Figure A.2 Representative cases of centerline scalar traces for completely unmixed scalars (solid line) and completely mixed scalars (dashed line). 
of $L / \lambda$ if a functional profile of the scalar diffusion layer is known or assumed. An error function profile will be used as the functional form of the scalar diffusion layer profile, where

$$
\phi(x)=\langle\phi\rangle+\frac{\Delta \phi}{2} \operatorname{erf}\left(\frac{x}{L}\right),
$$

$\Delta \phi=\phi_{1}-\phi_{2}$ and $\phi_{1}$ and $\phi_{2}$ are the scalar values of interest. This is reasonable as the error function represents the analytical solution to the scalar diffusion equation, where $L$ is typically defined as $L \equiv \sqrt{4 D t}$ and $D$ is the scalar diffusivity (Mills 1999). Broadwell and Briedenthal (1982) showed that a diffusion profile, under the influence of a strain field $S$, retains its error function profile, but the value of $L$ is modified such that $L^{\prime}=\sqrt{D /(2 S)}$. This is important to the present investigation, where the measured diffusion profiles occur in dynamically-evolving flows.

Figure A.3 shows the centerline density trace for a range of $L / \lambda$ values. This evolution of the density trace is physically equivalent to the perturbed surface never changing in form and scalar diffusion being the only dynamic mechanism. Figure A.4 shows the analytically calculated values of $B_{0}, B_{2}$, and $\theta$ for a range of $L / \lambda$ values. It is clearly seen that as the scalar $\phi$ diffuses, more mixed fluid is present at the centerline, and the value of $\theta$ increases as expected.

It is worth noting that a typical representation for the lengthscale of a diffusion profile is based upon a length between specified percentage values of a scalar concentration. This is typically referred to as a $5-95 \%$ or a $10-90 \%$ profile. The lengthscale used in the error function profile is equivalent to such a definition, but is inherently related to the physical process, rather than an arbitrary specification of the limits of the diffusion layer by an arbitrary set of percentages.

Also worthy of clarification is that the value of $L$ does not correspond to the complete span or width of the diffusion layer like a $5-95 \%$ profile does, but it does 


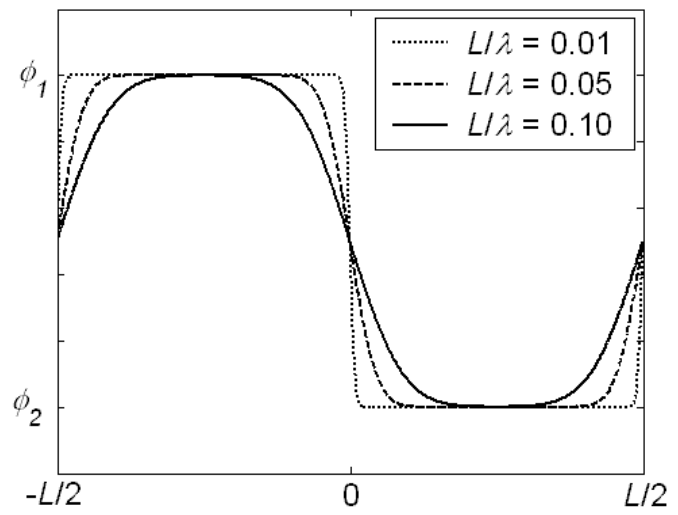

Figure A.3 Centerline scalar trace $\phi(x)$ for various values of $L / \lambda$.

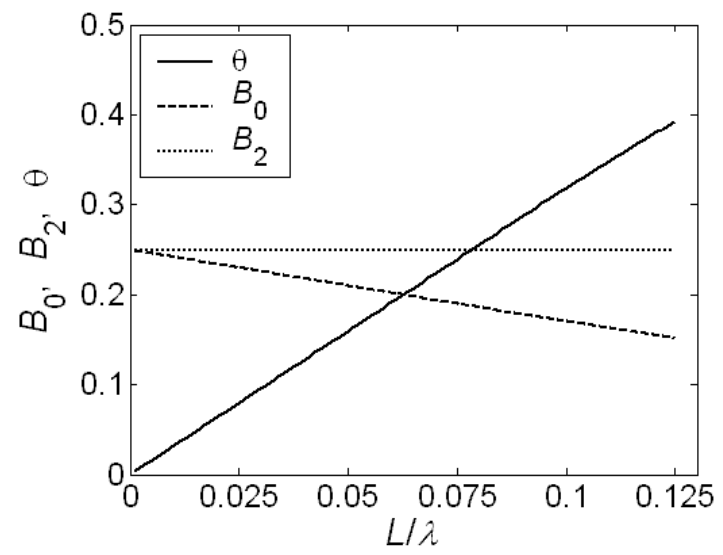

Figure A.4 Variation of $B_{0}, B_{2}$, and $\theta$ for a range of $L / \lambda$. 
represent the physically relevant lengthscale to the diffusion process. For this reason, the $L / \lambda$ values shown in figure A.4 range from $0<L / \lambda \leq 1 / 8$. Beyond $L / \lambda=1 / 8$, the scalar trace at the centerline no longer exhibits the minimum and maximum values of $\phi_{1}$ and $\phi_{2}$. However, it is worth noting that a value of $L / \lambda=1 / 8$ is not a physical limit to the examination of this problem, but it does represent a limit to the simplicity of the current investigation. A value $L / \lambda=1 / 8$ represents a bifurcation point, above which the flow may still contain a simple, single perturbation and exhibit a large value of $\theta$; however, typically the timescale of the evolution of the perturbation is much smaller than the timescale of the diffusion process. This implies that $\theta$ values larger than $\theta=0.4$ are more likely attributable to a complex, chaotic, or turbulent flow and not the fact that $L / \lambda>1 / 8$. This is evident in scalar traces that do contain the minimum and maximum values at the limits of $\phi_{1}$ of $\phi_{2}$, yet has a molecular mixing fraction of $\theta>0.4$. This is typically seen in mixing layers where the flow at the centerline is turbulent, yet pockets of unmixed fluids are still engulfed into the mixing layer and exist in their unmixed state at the centerline.

The relationship between $B_{0}, B_{2}$, and $\theta$ and $L / \lambda$ is shown in figure A.4. In simple flows with a single-mode perturbation, the measurement of $\lambda$ is relatively simple; however, the measurement of $L$ more difficult due the lengthscale of the diffusion layer involved. In the limit of $t \rightarrow 0$, the width of the diffusion layer approaches zero, which implies that the probe volume size required to fully resolve the diffusion layer must go to zero as well. Also, in strong strain fields, $L$ can be reduced to a value less than that predicted by the diffusivities of the scalars involved, requiring even smaller probes. Thus, for accurate scalar mixing measurements during the initial stages of mixing, an understanding of the required probe volume size is needed.

If the functional profile of the actual diffusion layer is known to be an error function profile, then the only parameter that determines the width of the diffusion 
layer is the lengthscale, $L$. Suppose that the actual and the measured diffusion profiles have the functional form

$$
\phi_{i}(x)=\langle\phi\rangle+\frac{\Delta \phi}{2} \operatorname{erf}\left(\frac{x}{L_{i}}\right)
$$

where $i=a$ for the actual profile and $i=m$ for the measured profile. This is the same as equation (A.1), except that the lengthscales for the actual and measured profiles are uniquely identified.

Given a scalar probe radius, $r$, the response of the probe to a given scalar field $\phi_{a}$ is the convolution of the actual scalar field with the response function of the measurement probe (Wilson 2002):

$$
\phi_{m}(x)=\int_{-\infty}^{\infty} \phi_{a}\left(x-x^{\prime}\right) R(x) d x^{\prime}
$$

where

$$
R(x)=\left\{\begin{array}{lll}
\frac{1}{2 r} \mathrm{e}^{x / r} & \text { if } & x<0 \\
\frac{1}{2 r} \mathrm{e}^{-x / r} & \text { if } & x \geq 0
\end{array}\right.
$$

is the response function of the probe, defined as such that the integral of $R(x)$ over the range $-\infty$ to $\infty$ equals unity. Figure A.5 shows the response function of the scalar probe and figure A.6 shows the results of the spatial averaging performed by the use of a probe with a finite probe volume radius.

The value of $L$ can be determined by measuring the slope of the diffusion profile at the inflection point, or at $x=0$. Taking the derivative of $\phi_{a}$ and $\phi_{m}$ at $x=0$ and rearranging gives

$$
L_{i}=\left(\left.\frac{\sqrt{\pi}}{2} \frac{\partial \phi_{i}}{\partial x}\right|_{x=0}\right)^{-1},
$$

where $i=a, m$. Consequently, a relationship between the slopes of the diffusion profiles at their inflection points can be determined and the measured slope can be corrected to obtain the actual diffusion lengthscale. Such a relationship can be derived 


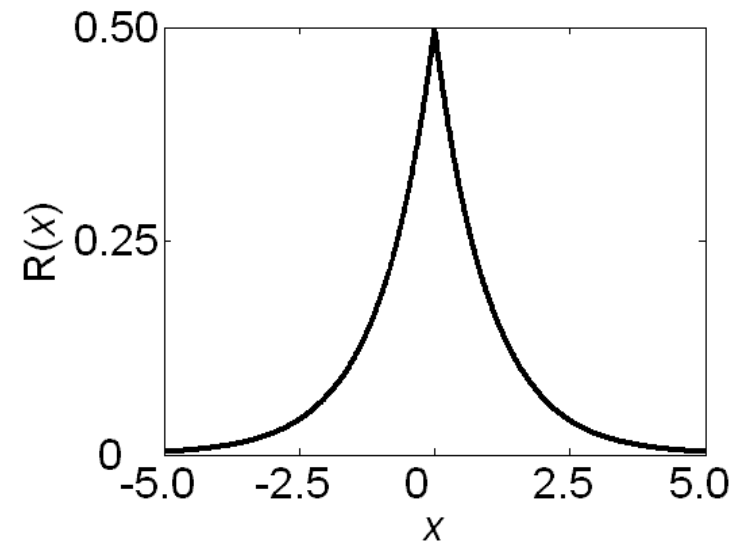

Figure A.5 Thermocouple probe volume response function for a probe radius $r=1$.

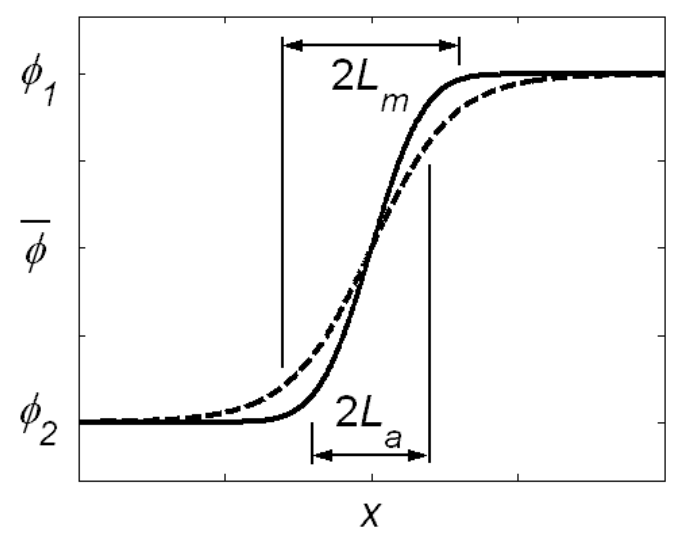

Figure A.6 The resulting centerline scalar trace (dashed line) and actual scalar trace (solid line). 
by integrating equation (A.3) to determine the measured profile.

Substituting equations (A.2) and (A.4) into equation (A.3) gives

$$
\begin{aligned}
\phi_{m}(x)= & \int_{-\infty}^{0}\left[\langle\phi\rangle+\frac{\Delta \phi}{2} \operatorname{erf}\left(\frac{x-x^{\prime}}{L_{a}}\right)\right] \frac{1}{2 r} \mathrm{e}^{x^{\prime} / r} d x^{\prime} \\
& +\int_{0}^{\infty}\left[\langle\phi\rangle+\frac{\Delta \phi}{2} \operatorname{erf}\left(\frac{x-x^{\prime}}{L_{a}}\right)\right] \frac{1}{2 r} \mathrm{e}^{-x^{\prime} / r} d x^{\prime}
\end{aligned}
$$

Since the response function integrates to a value of unity, the above expression simplifies to

$$
\begin{aligned}
\phi_{m}(x)= & \langle\phi\rangle+\frac{\Delta \phi}{4 r} \int_{-\infty}^{0} \operatorname{erf}\left(\frac{x-x^{\prime}}{L_{a}}\right) \mathrm{e}^{x^{\prime} / r} d x^{\prime} \\
& +\frac{\Delta \phi}{4 r} \int_{0}^{\infty} \operatorname{erf}\left(\frac{x-x^{\prime}}{L_{a}}\right) \mathrm{e}^{-x^{\prime} / r} d x^{\prime}
\end{aligned}
$$

The two integrals in equation (A.7) are computed as

$$
\begin{aligned}
\int_{-\infty}^{0} \operatorname{erf}\left(\frac{x-x^{\prime}}{L_{a}}\right) \mathrm{e}^{x^{\prime} / r} d x^{\prime} & =\mathrm{e}^{\eta_{a}^{2}} \mathrm{e}^{x / r} r \operatorname{erf}\left(\frac{x}{L_{a}}+\eta_{a}\right)+r \operatorname{erf}\left(\frac{x}{L_{a}}\right) \\
\int_{0}^{\infty} \operatorname{erf}\left(\frac{x-x^{\prime}}{L_{a}}\right) \mathrm{e}^{-x^{\prime} / r} d x^{\prime} & =-\mathrm{e}^{\eta_{a}^{2}} \mathrm{e}^{-x / r} r \operatorname{erf}\left(\frac{x}{L_{a}}-\eta_{a}\right)-r \operatorname{erf}\left(\frac{x}{L_{a}}\right),
\end{aligned}
$$

where $\eta_{a}=L_{a} / 2 r$. Substituting equation A.8 into equation A.7 and simplifying gives

$$
\phi_{m}(x)=\phi_{a}(x)+A(x)
$$

where equation (A.9) is the resulting convolution of the actual scalar profile with the scalar probe response function. The term $A(x)$ is responsible for the spatial averaging to the actual scalar trace and is defined

$$
A(x)=\frac{\Delta \phi}{2} \mathrm{e}^{\eta_{a}^{2}}\left[\sinh \left(\frac{x}{r}\right)-\frac{\mathrm{e}^{x / r}}{2} \operatorname{erf}\left(\frac{x}{L_{a}}+\eta_{a}\right)-\frac{\mathrm{e}^{-x / r}}{2} \operatorname{erf}\left(\frac{x}{L_{a}}-\eta_{a}\right)\right]
$$

With the convolution analytically evaluated, the relationship between the measured diffusion lengthscale and the actual diffusion lengthscale can be determined. 
Taking the inverse of equation (A.5) and subtracting the actual lengthscale from the measured lengthscale gives

$$
\frac{1}{L_{m}}-\frac{1}{L_{a}}=\left.\frac{\sqrt{\pi}}{2}\left(\frac{\partial \phi_{m}}{\partial x}-\frac{\partial \phi_{a}}{\partial x}\right)\right|_{x=0} .
$$

From equation (A.9),

$$
\phi_{m}(x)-\phi_{a}(x)=A(x)
$$

Substituting equation (A.12) into equation (A.11) yields

$$
\frac{1}{L_{m}}-\frac{1}{L_{a}}=\left.\frac{\sqrt{\pi}}{2} \frac{\partial A}{\partial x}\right|_{x=0}
$$

Evaluating $\partial A / \partial x$ at $x=0$ gives

$$
\left.\frac{\partial A}{\partial x}\right|_{x=0}=\frac{\Delta \phi}{2 r} \mathrm{e}^{\eta_{a}^{2}} \operatorname{erfc}\left(\eta_{a}\right)-\frac{\Delta \phi}{L_{a} \sqrt{\pi}},
$$

where $\operatorname{erfc}(x)=1-\operatorname{erf}(x)$ is the complementary error function. Thus, equation (A.13) becomes

$$
L_{m}=\left[\frac{\Delta \phi \sqrt{\pi}}{4 r} \mathrm{e}^{\eta_{a}^{2}} \operatorname{erfc}\left(\eta_{a}\right)-\frac{\Delta \phi}{2 L_{a}}+\frac{1}{L_{a}}\right]^{-1}
$$

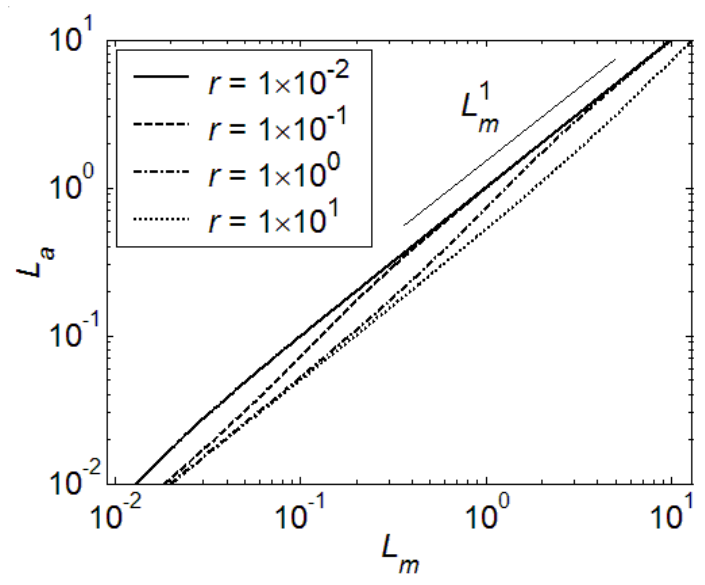

Figure A.7 Correction curves for $L_{m}$ with $r$ as a parameter. 
Equation (A.15) is the fundamental equation necessary for correcting the measured lengthscale, $L_{m}$, to the value of the actual lengthscale, $L_{a}$. The relationship $L_{a}=f\left(L_{m}\right)$ is not readily discernible, so the graphical relationship between $L_{a}$ and $L_{m}$ is shown in figure A.7. It can be seen in figure A.7 that when $r \ll L_{a}$, then $L_{a}=L_{m}$. This is expected, as the scalar probe is fully capable of resolving all spatial gradients. However, as $r$ approaches the value of $L_{a}$, the functional relationship $L_{a}=L_{m}$ breaks down and $L_{m}$ becomes greater than $L_{a}$. In this case, the need for correcting the spatially-averaged value of $L_{m}$ becomes necessary when the probe radius is of the same order of magnitude or greater than the diffusion lengthscale in question and is determined by equation (A.15). 


\section{APPENDIX B}

\section{THERMOCOUPLE NOISE REDUCTION}

Temperature measurements in the water channel were made with small diameter $(0.08 \mathrm{~mm}) 40$ gauge wire thermocouples. The use of such small wires in an electricallyconducting medium amplifies the ambient electromagnetic interference (EMI) and radio-frequency interference (RFI) voltages that are sampled by the data acquisition (DAQ) system. This noise introduces fluctuating voltages, due to aliasing effects, within the spectral range of interest for this investigation. Thus, the use of highand low-pass filtering techniques is not available for eliminating this noise when postprocessing the data, and the noise must be mitigated at its source. Two steps were taken to control extraneous voltage readings by the thermocouple/DAQ system.

First, it was determined that the electrical interaction between two thermocouples in the water channel produced a "cross-talk" effect, resulting in voltage fluctuations being measured on each of the thermocouples being used. The original thermocouple configuration used in the water channel used two thermocouples and two DAQ systems. The first thermocouple was connected to an 8-bit DAQ system with $100 \mathrm{~Hz}$ sampling rate. The second thermocouple was connected to the same 16-bit DAQ system as described in $\S 2.2$. The two thermocouples were mounted on the same vertical rake and were positioned very close to one another. The first thermocouple was used for the detection of the centerplane of the mixing layer by measuring $\overline{f_{1}}$ as described by equation (2.6). Once the two thermocouples were located on the centerplane, the second thermocouple, which was connected to the new DAQ system, was used to take high-resolution temperature measurements at $100 \mathrm{kHz}$.

The spurious voltage fluctuations were eliminated by changing the configuration and eliminating the use of one thermocouple and the original DAQ system. In the new 
system, only one thermocouple was used to measure both the centerplane placement of the probe volume and the full resolution temperature measurements. However, the current DAQ software did not allow for the creation of data manipulation or averaging calculations. A program was written so that the following operating procedure could be used.

- First, the thermocouple would be placed at the approximate vertical location of the centerplane.

- Temperature measurements would be sampled at $200 \mathrm{~Hz}$ for approximately 50 seconds. Once the sampling was complete, the data would be written to disk under the filename "c: \center.dat".

- The program would be invoked to calculate $\overline{f_{1}}$ using a 10 -point window averaging routine to minimize spurious noise. If $\overline{f_{1}}=0.5 \pm 0.25$, then the next steps would be completed, otherwise, the thermocouple was repositioned accordingly and the previous step was repeated.

- Finally, once the thermocouple had been successfully located on the centerplane, temperature measurements would be recorded at $50 \mathrm{kHz}$.

In addition to eliminating all cross-talk voltage fluctuations, it was desired to eliminate all EMI- and RFI-induced voltage fluctuations. To accomplish this, the 40-gauge thermocouple wire was limited to 3 feet in length. The remainder of the wire connecting the DAQ system to the 40-gauge wire was replaced with 18-gauge, twisted and shielded E-type thermocouple extension wire. This minimizes the length over which the 40-gauge wire can act as an antenna for RFI and EMI noise. In addition, the 40-gauge wire was shielded with a thin layer of tin foil terminating approximately $2 \mathrm{~cm}$ before the probe volume. The shielding on both the 40-gauge 
and 18-gauge wires were grounded, effectively removing most of the RFI and EMI noise from the thermocouple system. 


\section{APPENDIX C}

\section{ENTRANCE PLENUM ENHANCEMENTS}

The combination of wire meshes placed within the entrance plenum of the water channel serve the purpose of eliminating free-stream turbulence fluctuations and minimizing the momentum deficit along the walls and splitter plate. Originally, the stainless steel meshes were caulked to $1 / 2$ " $\times 1 / 2$ " plastic "egg-crate" grills for support, as shown in figure C.1. The first enhancement made to the screen mesh/egg-crate combination was to attach the screens by melting the steel screen into the plastic egg-crate. In this manner, only the area on the screen mesh covered by the plastic egg-crate filled the holes in the screen mesh, whereas the caulking process occupied more screen mesh area than was necessary. The second enhancement was to remove the plastic ribs from the egg-crates that were closest to the splitter plate. It was found from dye visualization experiments that the ribs adjacent to the splitter plate created a recirculation region which tripped the formation of a turbulent boundary layer just downstream of the screen mesh. By removing this rib, the boundary layers

formed just downstream of the screen meshes were found to be smaller and laminar, as shown in figure C.2. 


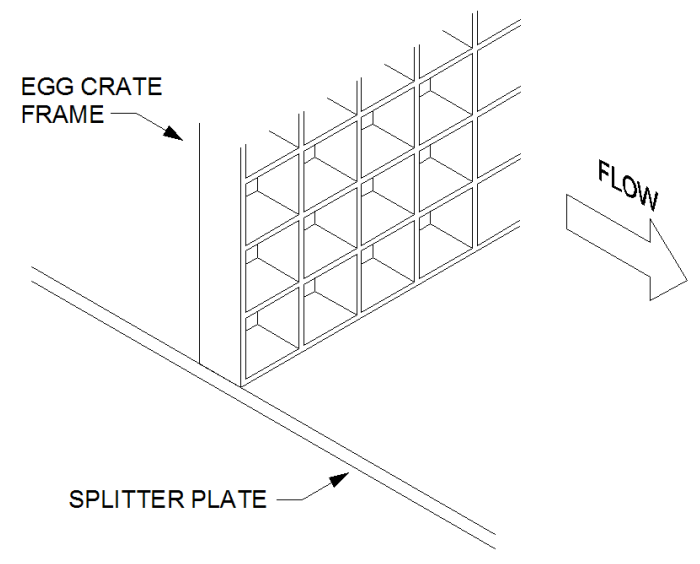

Figure C.1 Schematic of the entrance plenum showing egg-crate grill and splitter plate. The screen meshes are not shown.
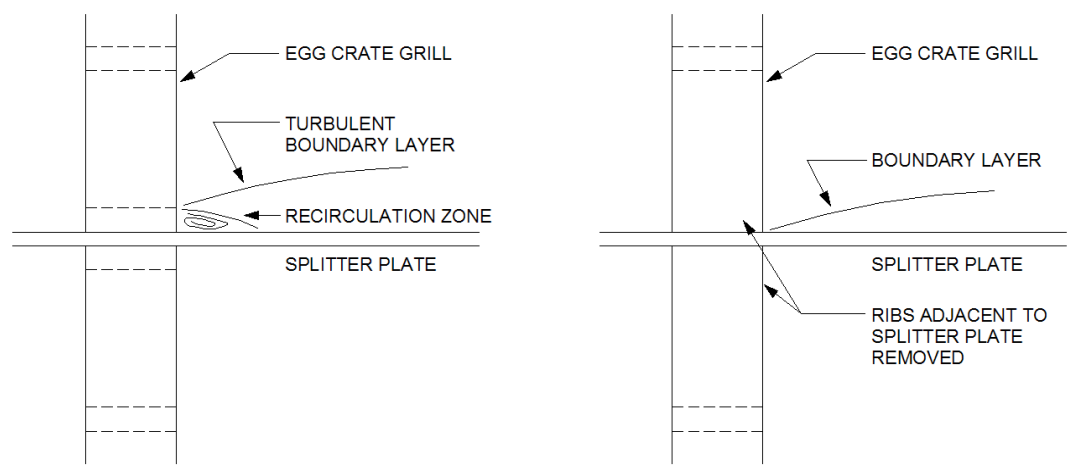

Figure C.2 Schematic of boundary layer growth before (left) and after (right) the removal of the plastic ribs adjacent to the splitter plate. 


\section{VITA}

Name:

Date and place of birth:

Permanent Address:

Education:
Nicholas Mueschke

November 2, 1978

Houston, Texas

131 Ancestral Rd.

Carencro, LA 70520

B.S. (Mechanical Engineering), May 2002

University of Louisiana at Lafayette, Lafayette, LA

M.S. (Mechanical Engineering), December 2004

Texas A\&M University, College Station, TX 